\title{
LA-UR-17-20082
}

Approved for public release; distribution is unlimited.

Title: Mesoscopic modeling of ferroic and multiferroic materials

Author(s): $\quad$ Saxena, Avadh

Intended for: General colloquium

Issued: 
Disclaimer:

Los Alamos National Laboratory, an affirmative action/equal opportunity employer, is operated by the Los Alamos National Security, LLC for the National Nuclear Security Administration of the U.S. Department of Energy under contract DE-AC52-06NA25396. By approving this article, the publisher recognizes that the U.S. Government retains nonexclusive, royalty-free license to publish or reproduce the published form of this contribution, or to allow others to do so, for U.S. Government purposes. Los Alamos National Laboratory requests that the publisher identify this article as work performed under the auspices of the U.S. Department of Energy. Los Alamos National Laboratory strongly supports academic freedom and a researcher's right to publish; as an institution, however, the Laboratory does not endorse the viewpoint of a publication or guarantee its technical correctness. 


\section{MESOSCOPIC MODELING OF FERROIC AND MULTIFERROIC MATERIALS}

\section{Avadh Saxena (Los Alamos National Lab)}

1. Strain: order parameter (symmetry determined).

2. Landau free energy; gradient energy.

3. Domains, microstructure and elastic compatibility.

4. Coupling of strain to polarization/magnetization; multiferroics.

5. Disorder: spin glass, relaxors, strain glass.

6. Bioferroics: Ferroic phenomena in biology.

Collaborators: T. Lookman, A. Planes, T. Castan, K. Otsuka, X. Ren. 


\section{Three Pillars of Functional Materials/Devices}

\section{CHARGE \\ SPIN \\ LATTICE}

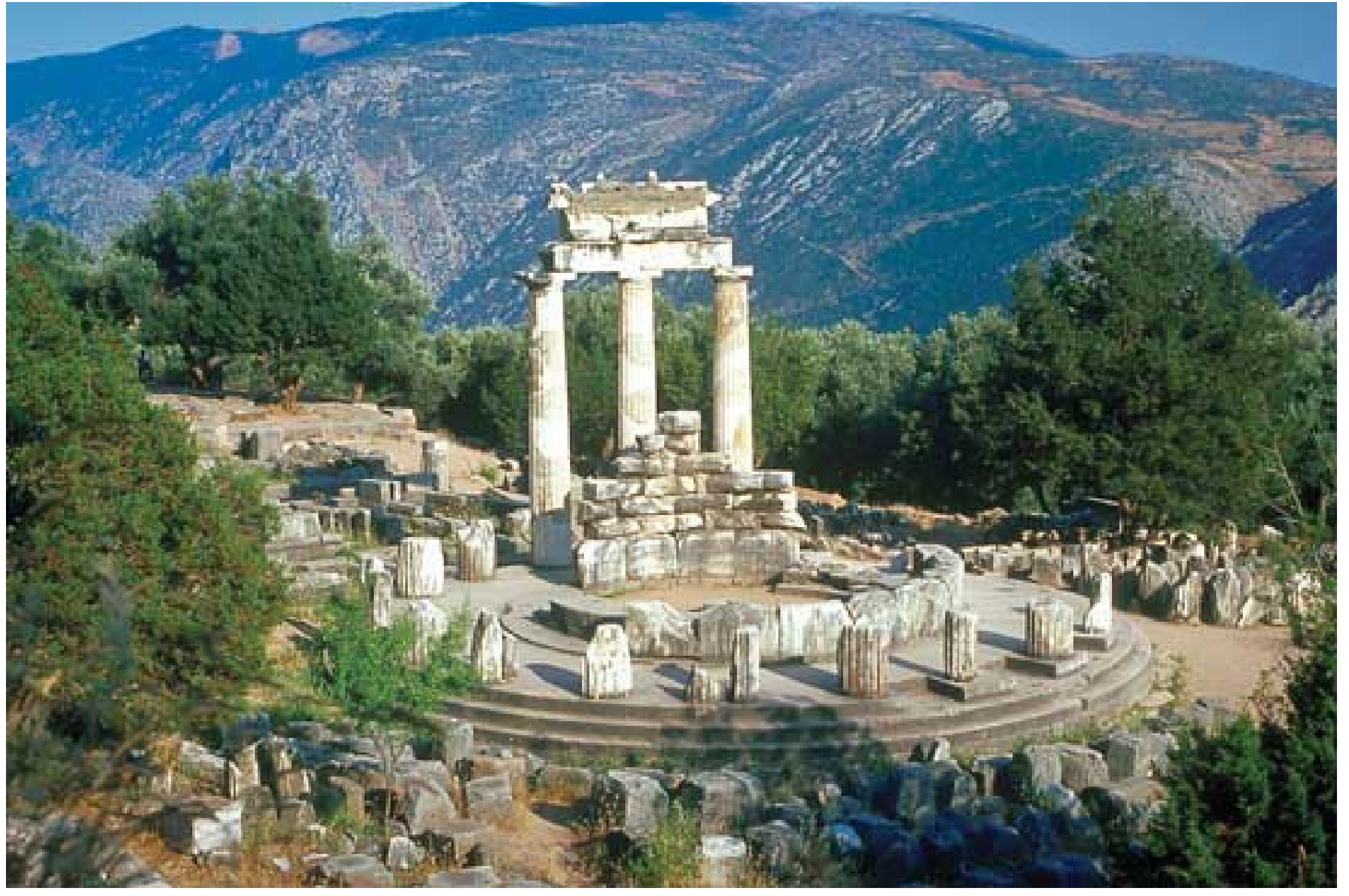

Tholos Temple at Delphi, Greece, 300 BC 


\section{FERROIC MATERIALS}

Ferroics: Family of materials exhibiting one or more functional characteristics such as magnetization (in ferromagnetic materials), polarization (in ferroelectric materials), strain (in ferroelastic materials), .... which occur at a (often first-order) phase transition.

\section{Features:}

1. DOMAINS occur at the transition.

2. Functional properties can be SWITCHED by an external conjugate field.

3. They show HYSTERESIS (and thus spontaneous order at zero field).

4. They can be utilized as SMART materials.

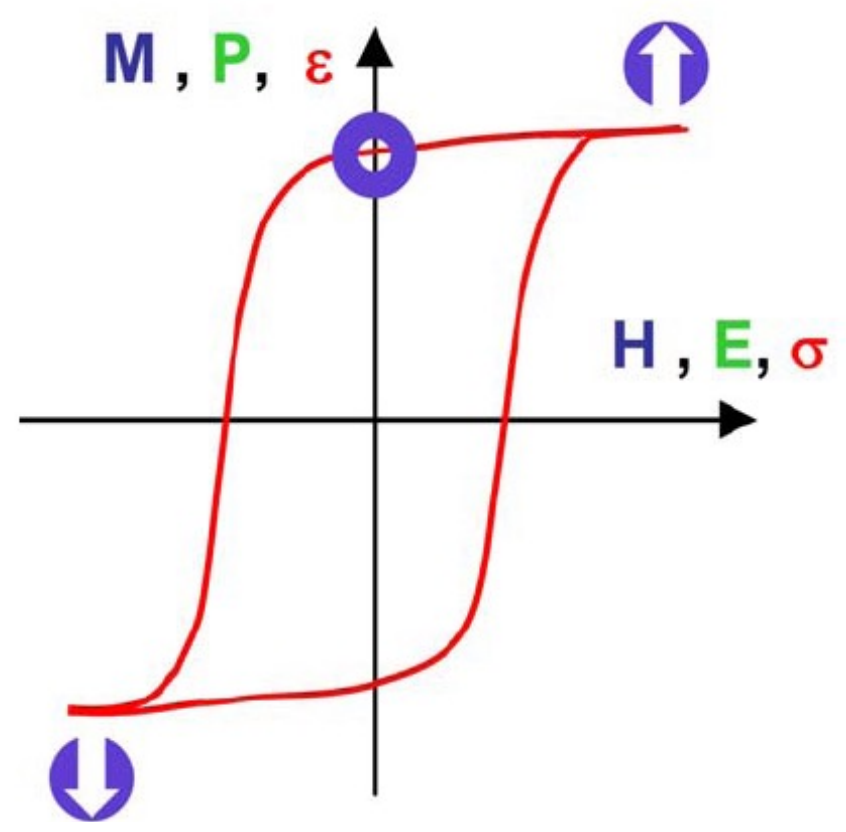




\section{Four Primary Ferroics: Symmetry}

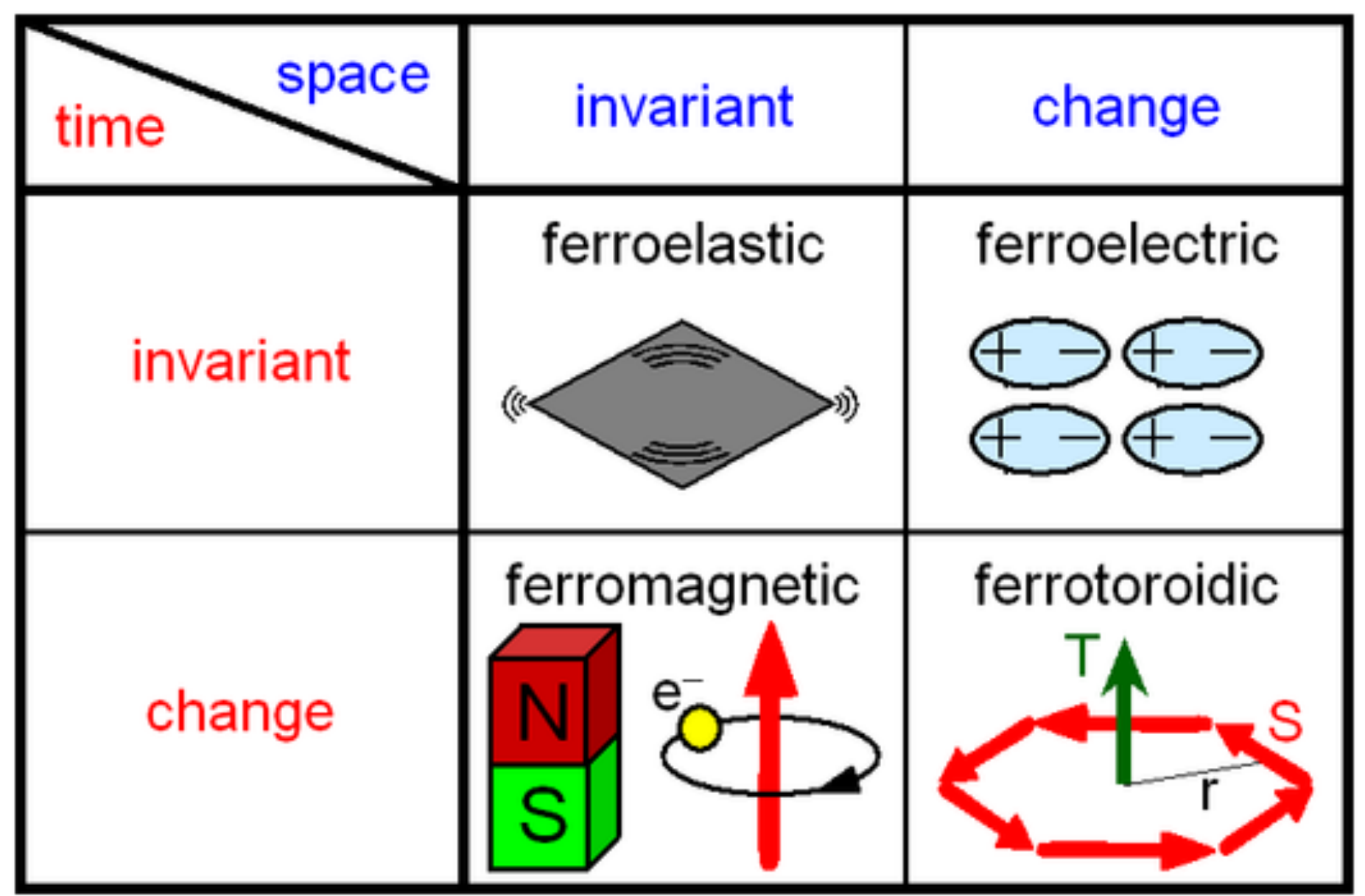




\section{Ferroics with vector order parameter}

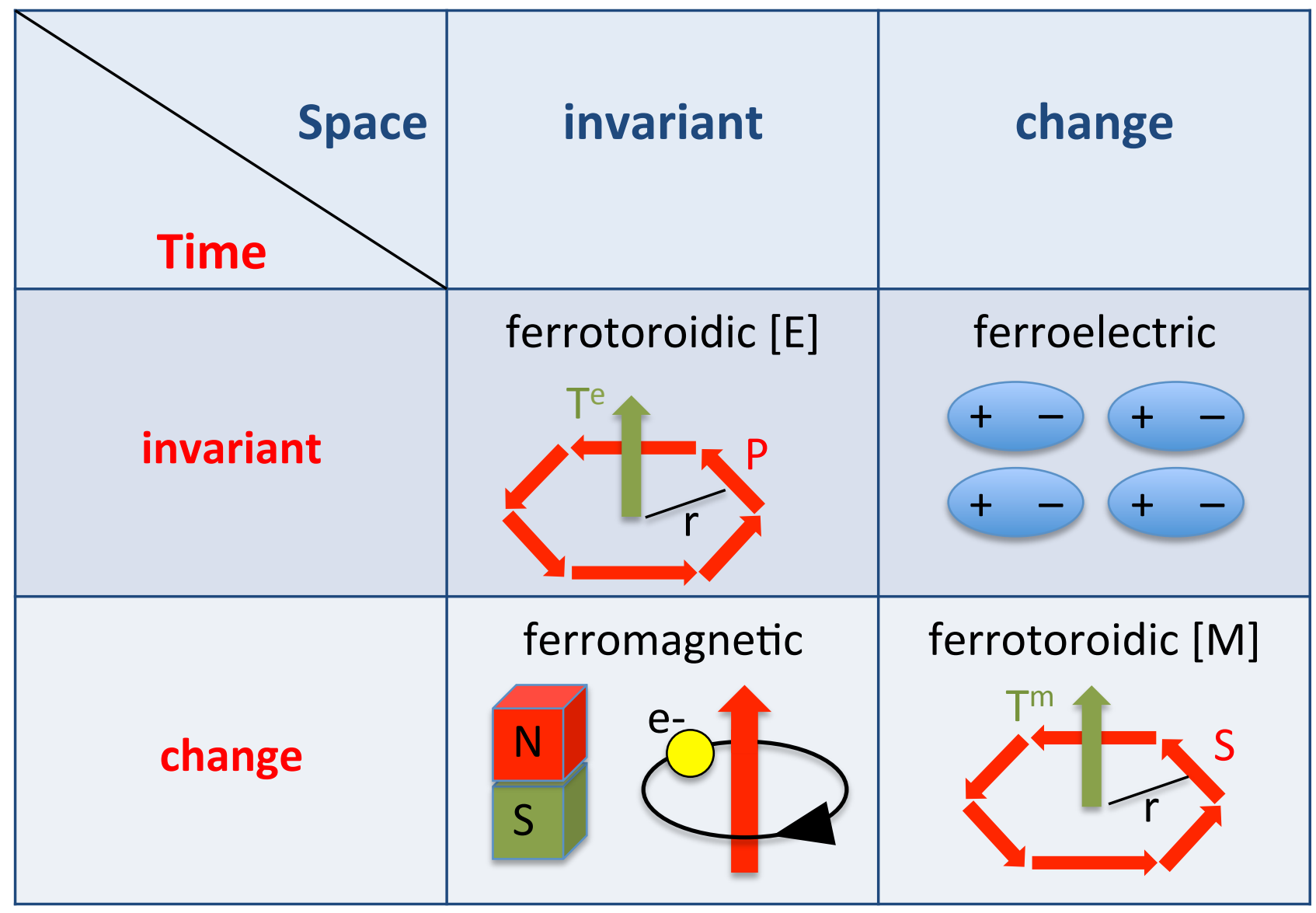




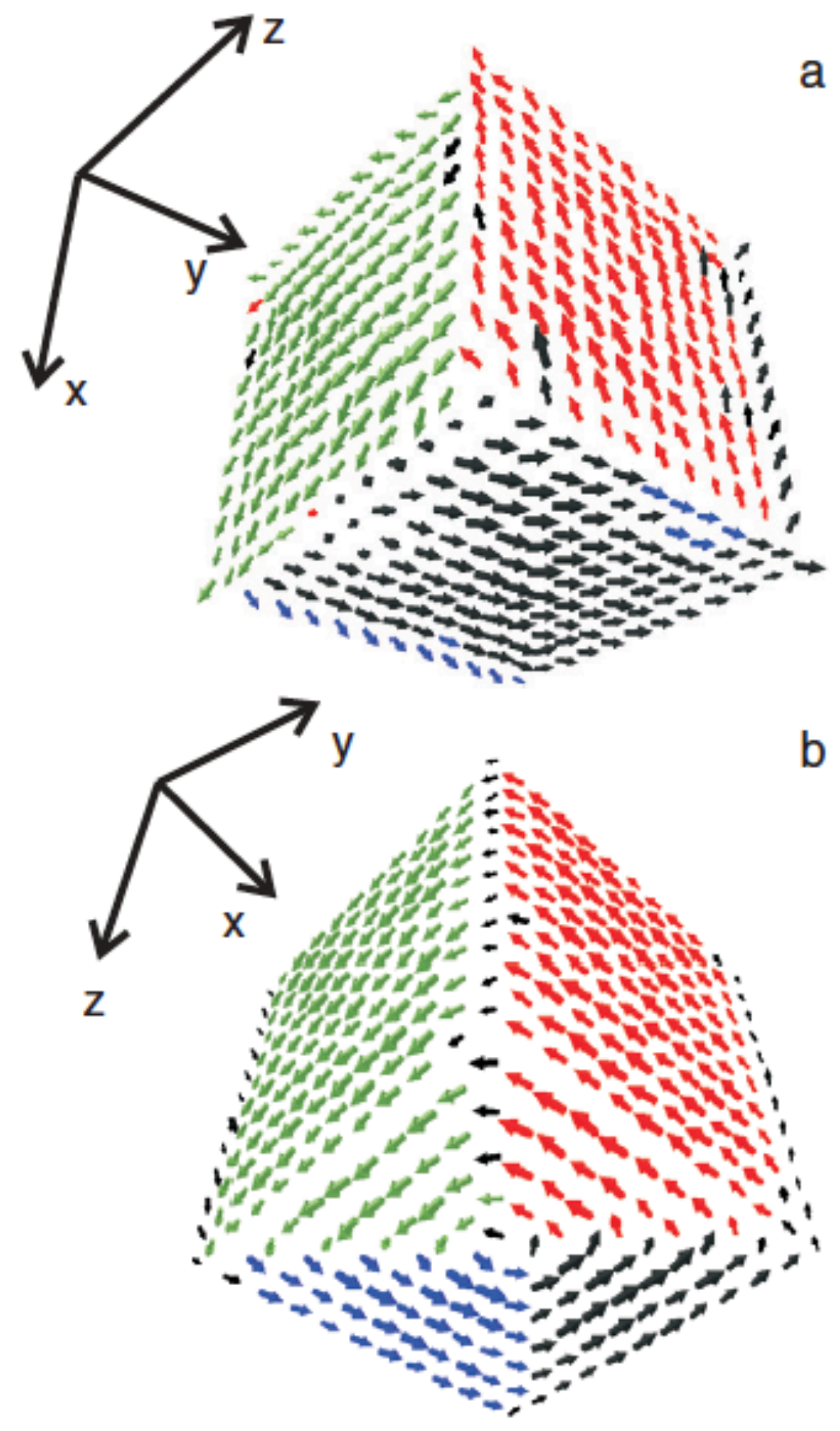

\section{Dipolar vortices}

a

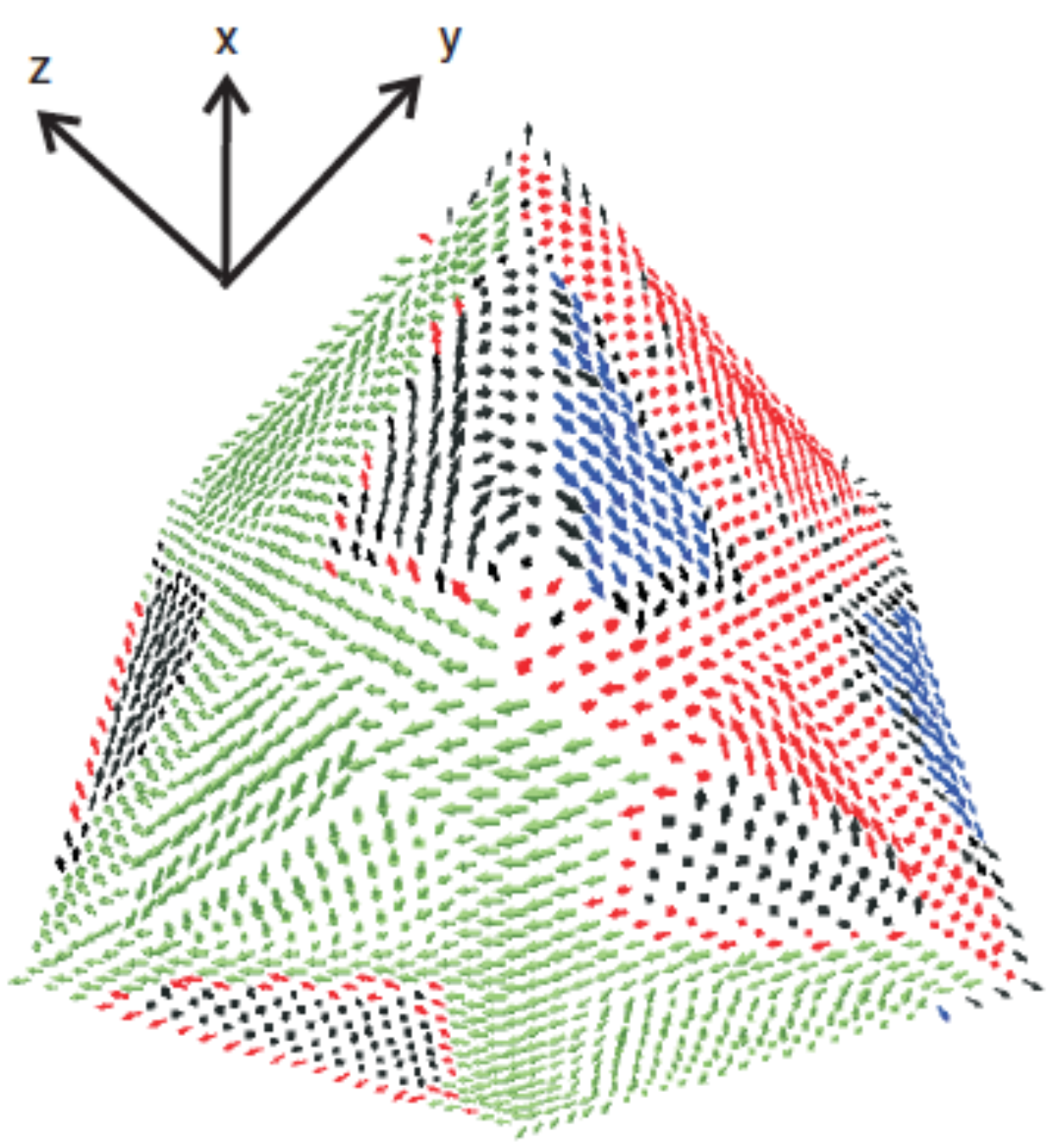

S. Prosandeev et al. (2008) 


\section{DIPOLE MOMENT (vector)}

A $\oplus$

B

C
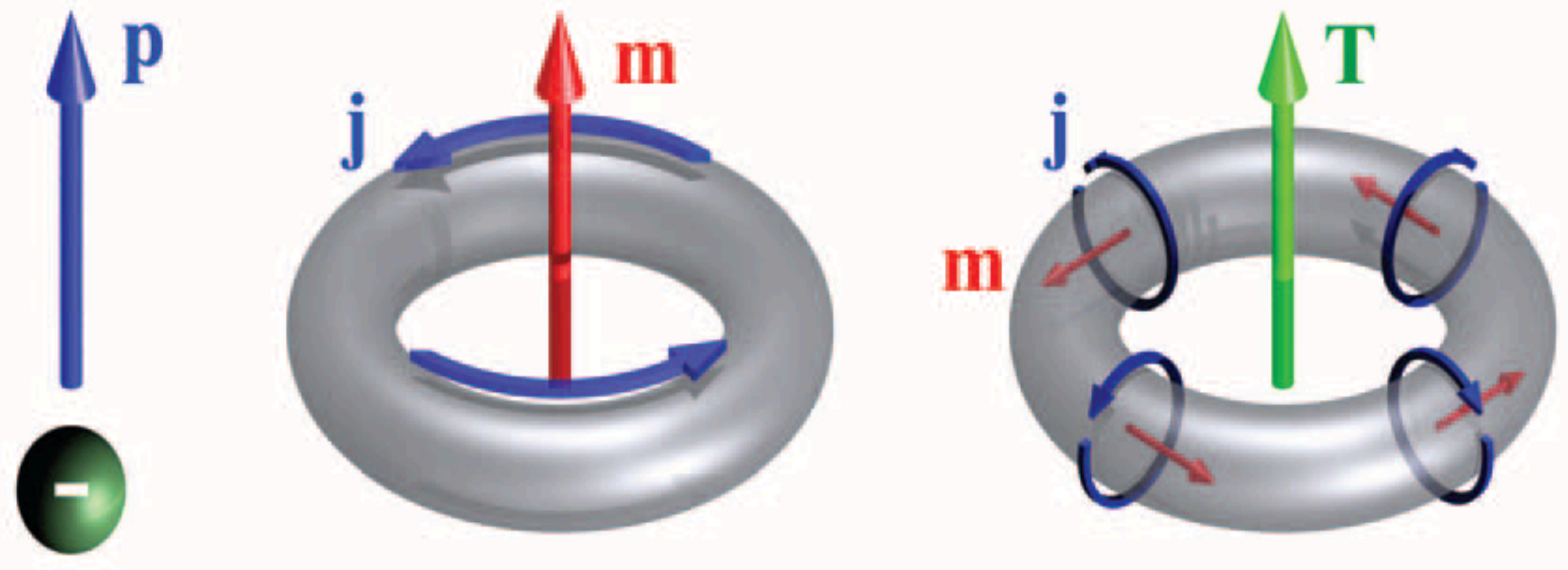

ELECTRIC (polar) MAGNETIC (axial)

TOROIDAL (axio-polar)

$\sim 1 / r^{3}$

$\sim 1 / r^{3}$

$\sim 1 / r^{5}$ 


\title{
HYPERTOROIDAL MOMENT
}
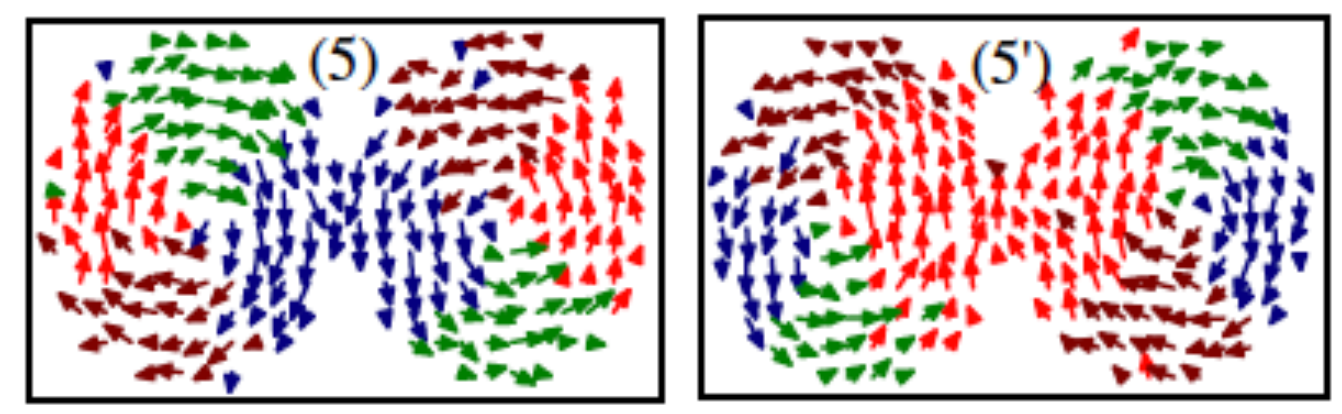

\author{
ELECTRIC
}
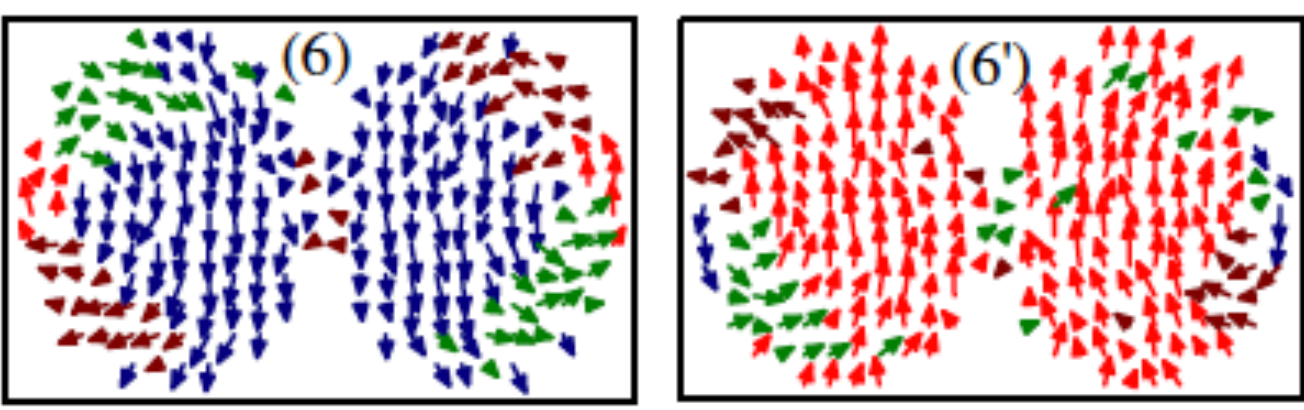

Double vortices

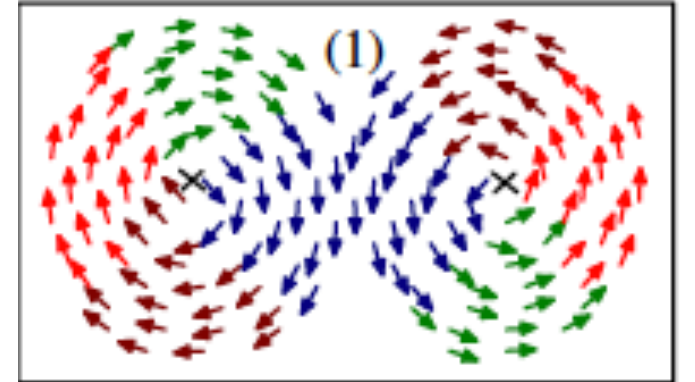

MAGNETIC

S. Prosandeev and L. Bellaiche J. Mater. Sci. 44, 5235 (2009).
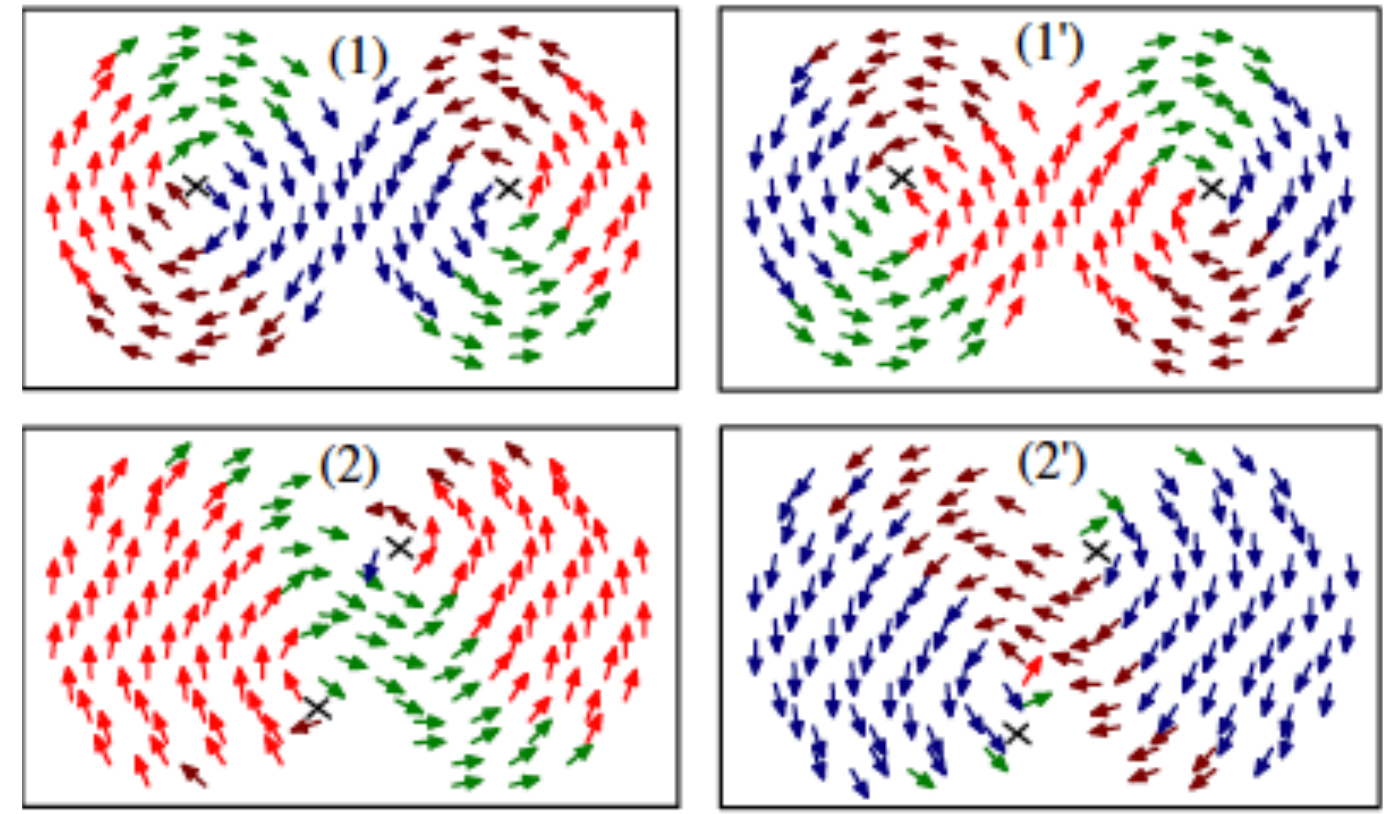


\section{ELASTIC DIPOLE MOMENT (polar tensor)}
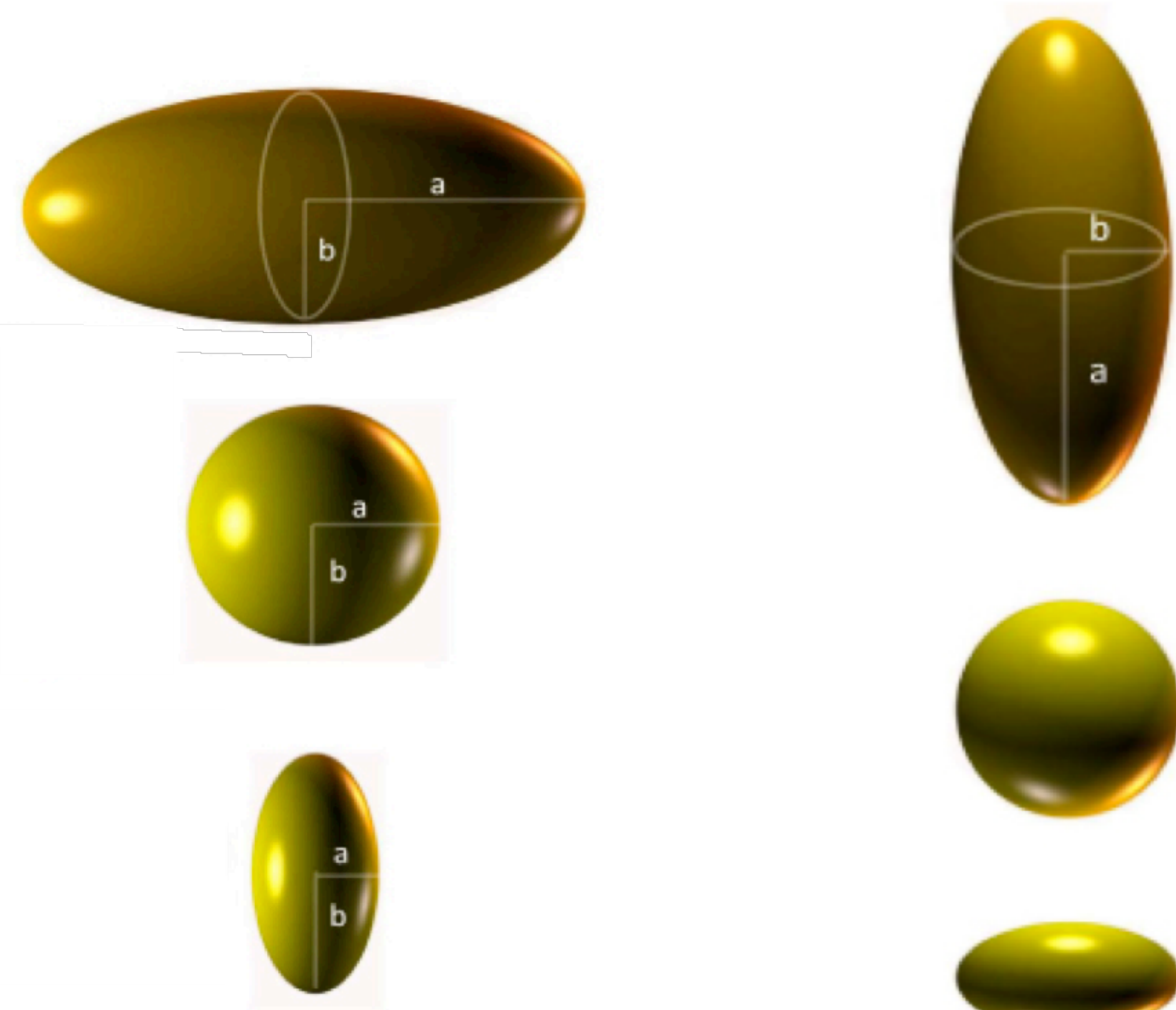

DEVIATION FROM SPHERICITY $\left(\sim 1 / r^{5}\right)$ 


\section{FERROIC PROPERTY QUADRANGLE}

\begin{tabular}{|c|c|c|c|c|}
\hline FERROICS & Property & Precursor & Glass & Caloric \\
\hline Ferromagnetic & $\mathrm{M}$ & $\begin{array}{c}\text { Magnetic } \\
\text { tweed }\end{array}$ & $\begin{array}{c}\text { Spin } \\
\text { glass }\end{array}$ & Magnetocaloric \\
\hline Ferroelectric & $\mathrm{P}$ & $\begin{array}{c}\text { Polar } \\
\text { tweed }\end{array}$ & $\begin{array}{c}\text { Polar } \\
\text { glass }\end{array}$ & Electrocaloric \\
\hline Ferroelastic & $\mathrm{e}$ & Tweed & $\begin{array}{c}\text { Strain } \\
\text { glass }\end{array}$ & Elastocaloric \\
\hline Ferrotoroidic & $\mathrm{T}$ & $\begin{array}{c}\text { Toroidic } \\
\text { tweed }\end{array}$ & $\begin{array}{c}\text { Toroidic } \\
\text { glass }\end{array}$ & Toroidocaloric \\
\hline
\end{tabular}
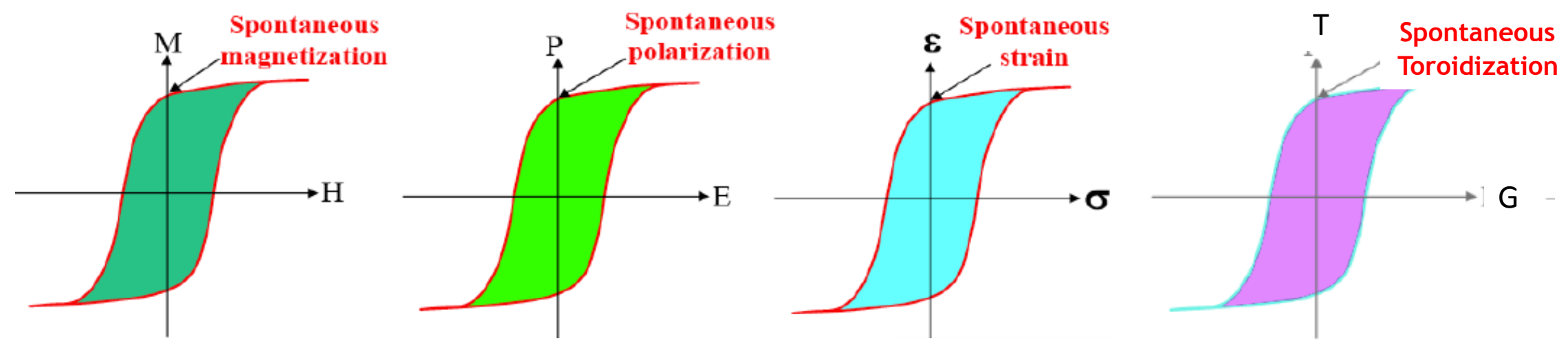


\section{SYMMETRY, FREE ENERGY MICROSTRUCTURE}




\section{Elements of Landau Theory}

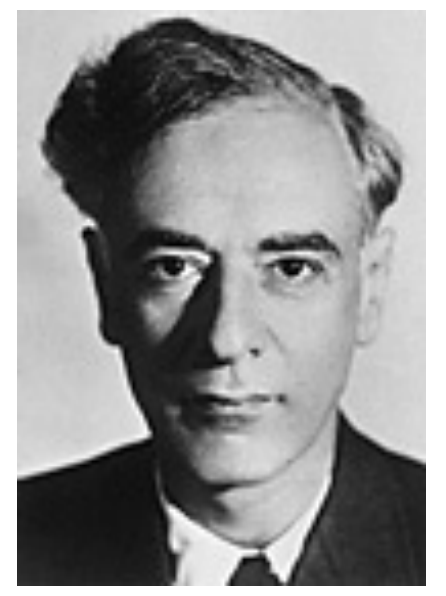

Lev Davidovich Landau 1908-1968

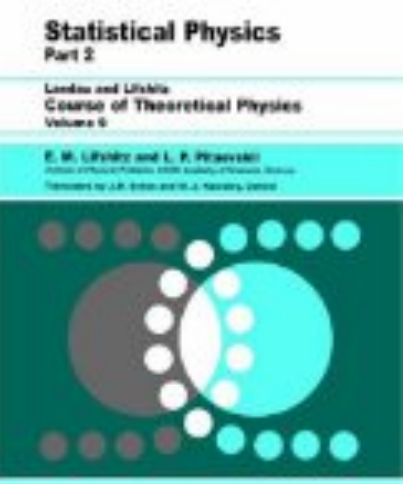

- Symmetry-based treatment

- Introduces formally the Order Parameter.

- Starting point: in the vicinity of the phase transition the free energy can be expanded in powers of the Order Parameter.

- The expansion includes only terms which are allowed by symmetry. 


\section{LANDAU THEORY}

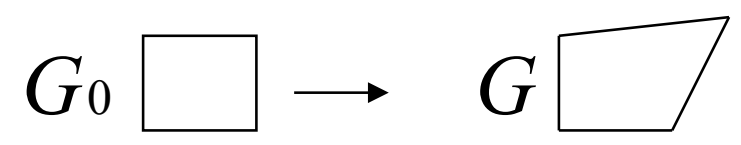

1. Distortion:

$$
\Delta \rho(r)=\rho_{0}(r)-\rho(r)
$$

2. Strain:

3. Free energy:

$$
\begin{aligned}
\square & \rightarrow \square \\
\Delta \rho(r) & =\sum C_{i j} \varepsilon_{i j} \\
& =\eta_{1} e_{1}+\eta_{2} e_{2}+\eta_{3} e_{3}
\end{aligned}
$$

4. Truncation:

$$
F[\delta \rho] \rightarrow F\left[e_{1}, e_{2}, e_{3}\right] \stackrel{\mathrm{OP}}{\longrightarrow} F\left[e_{3}\right]
$$

$$
\begin{aligned}
& F\left[e_{3}\right]=a(P, T) e_{3}{ }^{2}+b e_{3}{ }^{3}+c e_{3}{ }^{4} \\
& F\left[e_{3}\right]=a(P, T) e_{3}{ }^{2}+b e_{3}{ }^{4}+c e_{3}{ }^{6} \\
& F\left[e_{3}\right]=a(P, T) e_{3}{ }^{2}+c e_{3}{ }^{4}
\end{aligned}
$$

$$
\begin{aligned}
& e_{1}=\varepsilon_{x x}+\varepsilon_{y y} \\
& e_{2}=\varepsilon_{x y} \\
& e_{3}=\varepsilon_{x x}-\varepsilon_{y y}
\end{aligned}
$$




\section{Relationship with Theory and Experiments}

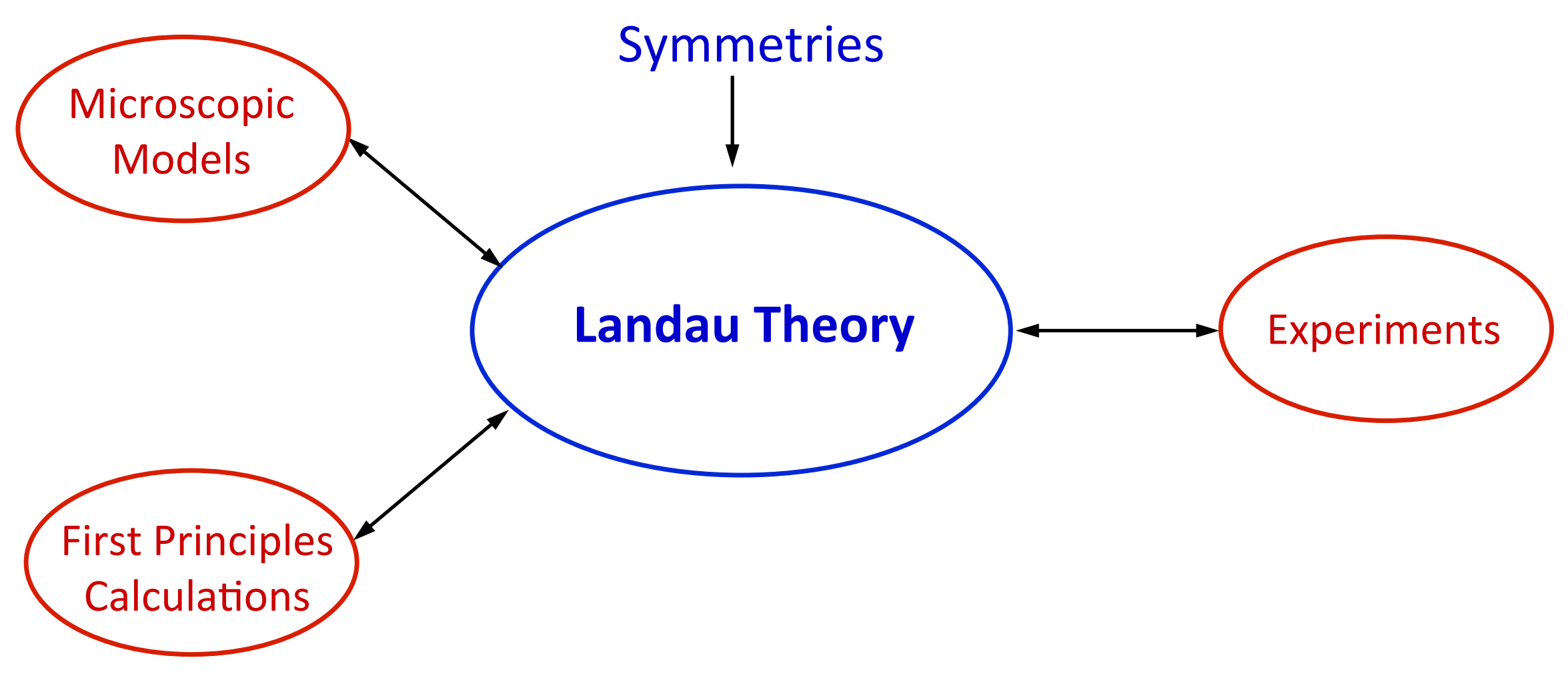




\section{Microstructure for Structural Transitions}

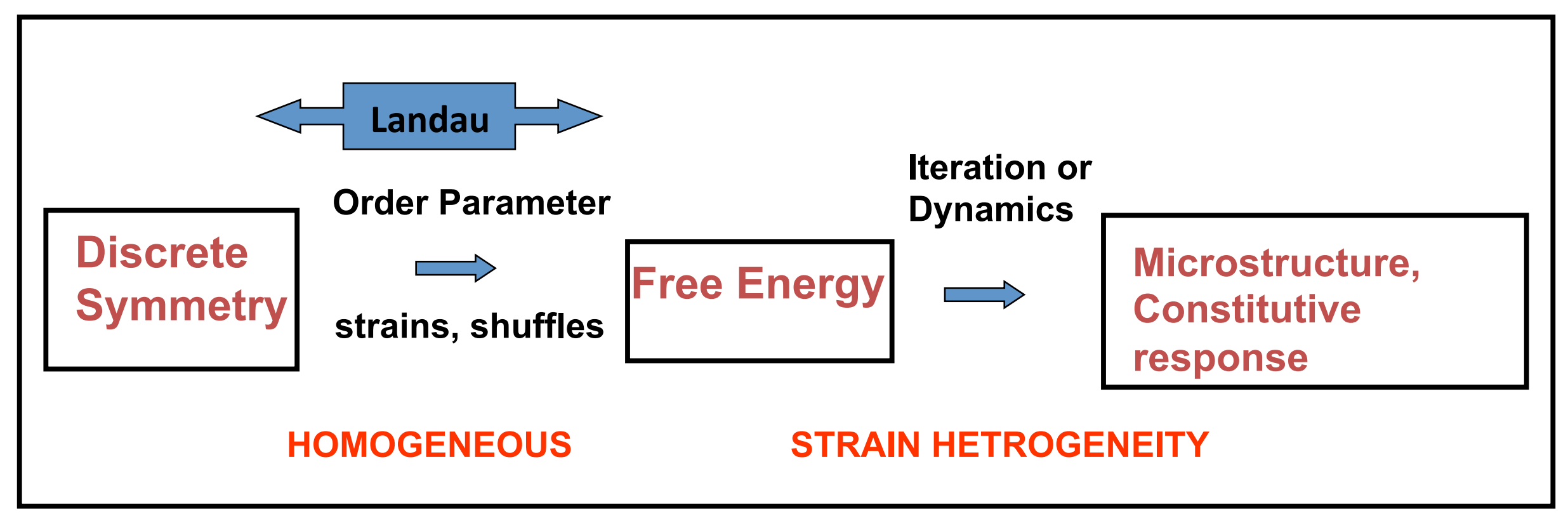




\section{Order Parameters}

\section{Functionalities $\varepsilon, P, M, \psi$}
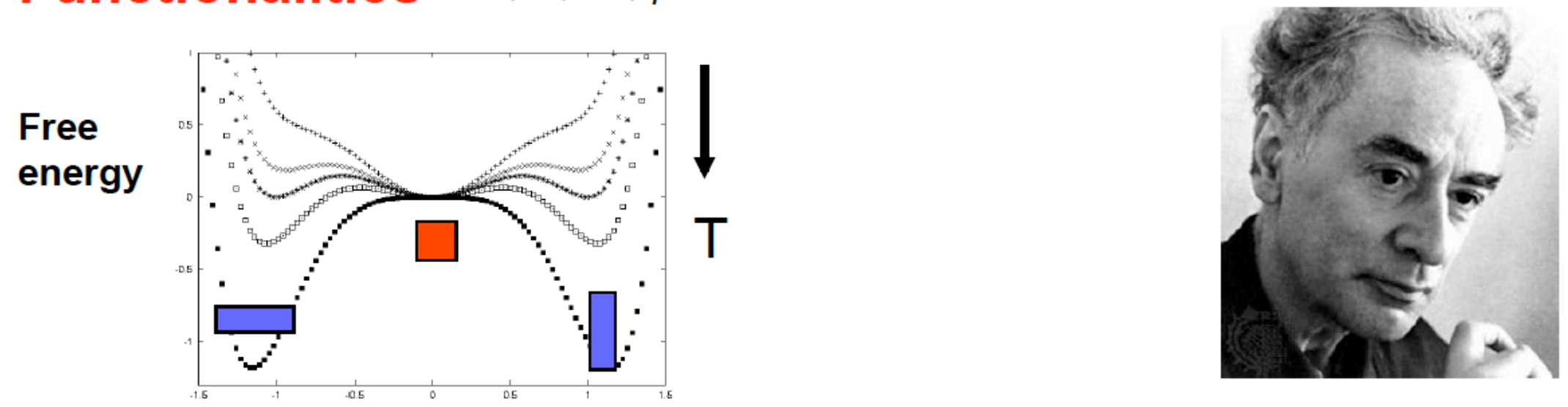

Landau, 1937

\section{$+$}

Spatial variations
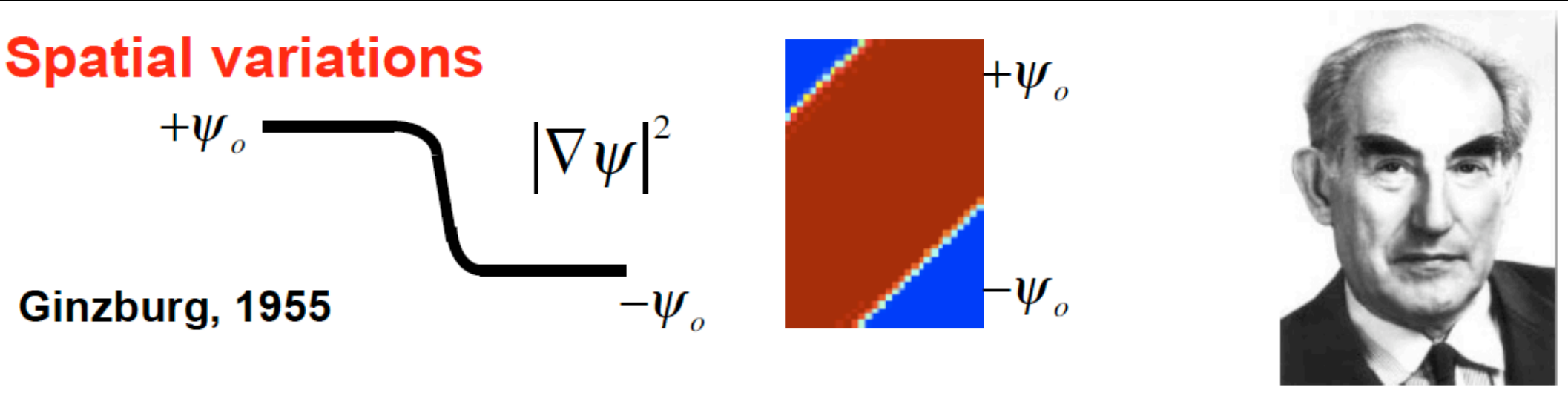

Free Energy : $F(\varepsilon, P, M, \psi: \nabla \varepsilon, \nabla \psi .$. 


\section{Adhemar Jean Claude Barre de Saint-Venant}

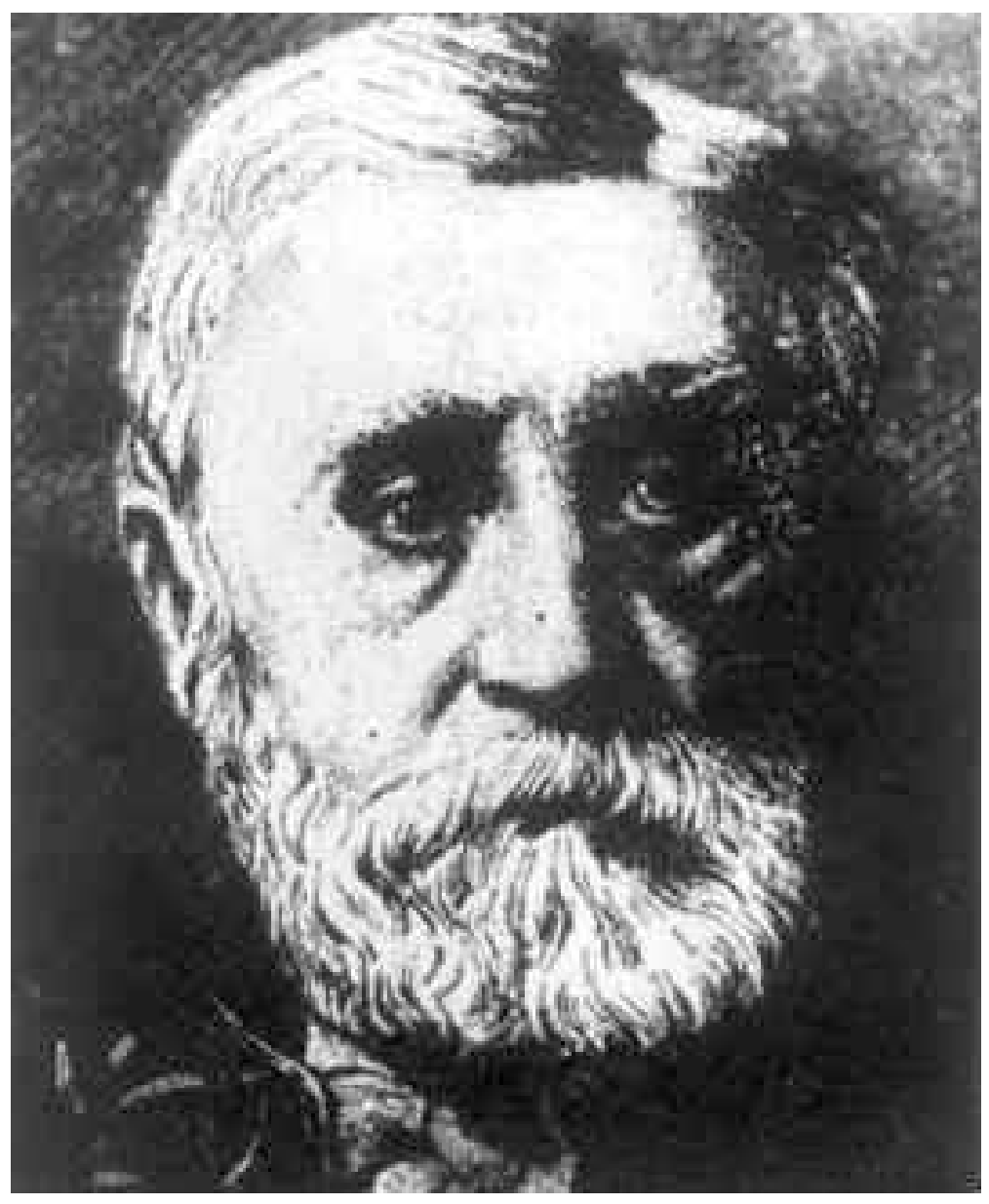

$1797-1886$ 


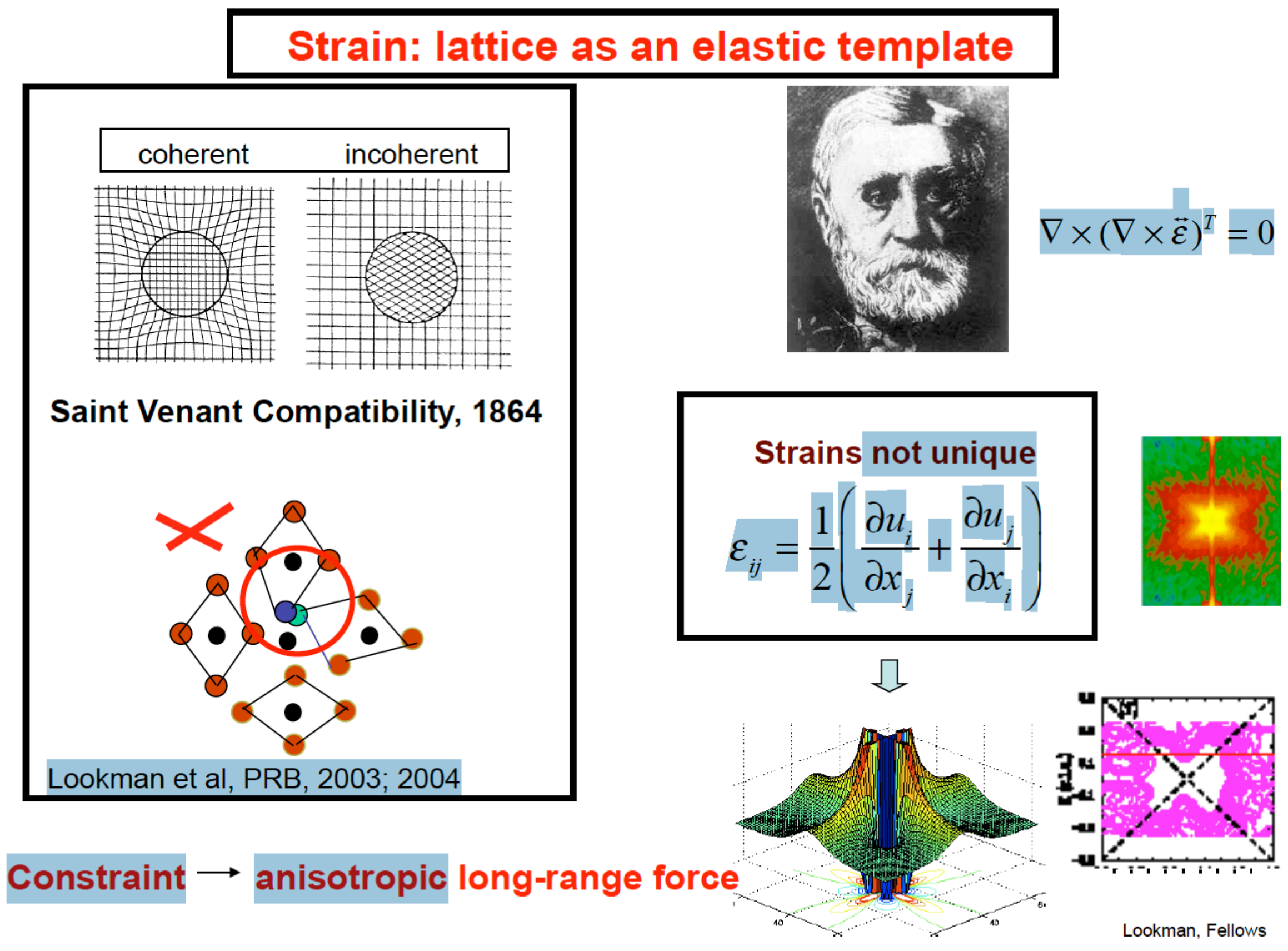



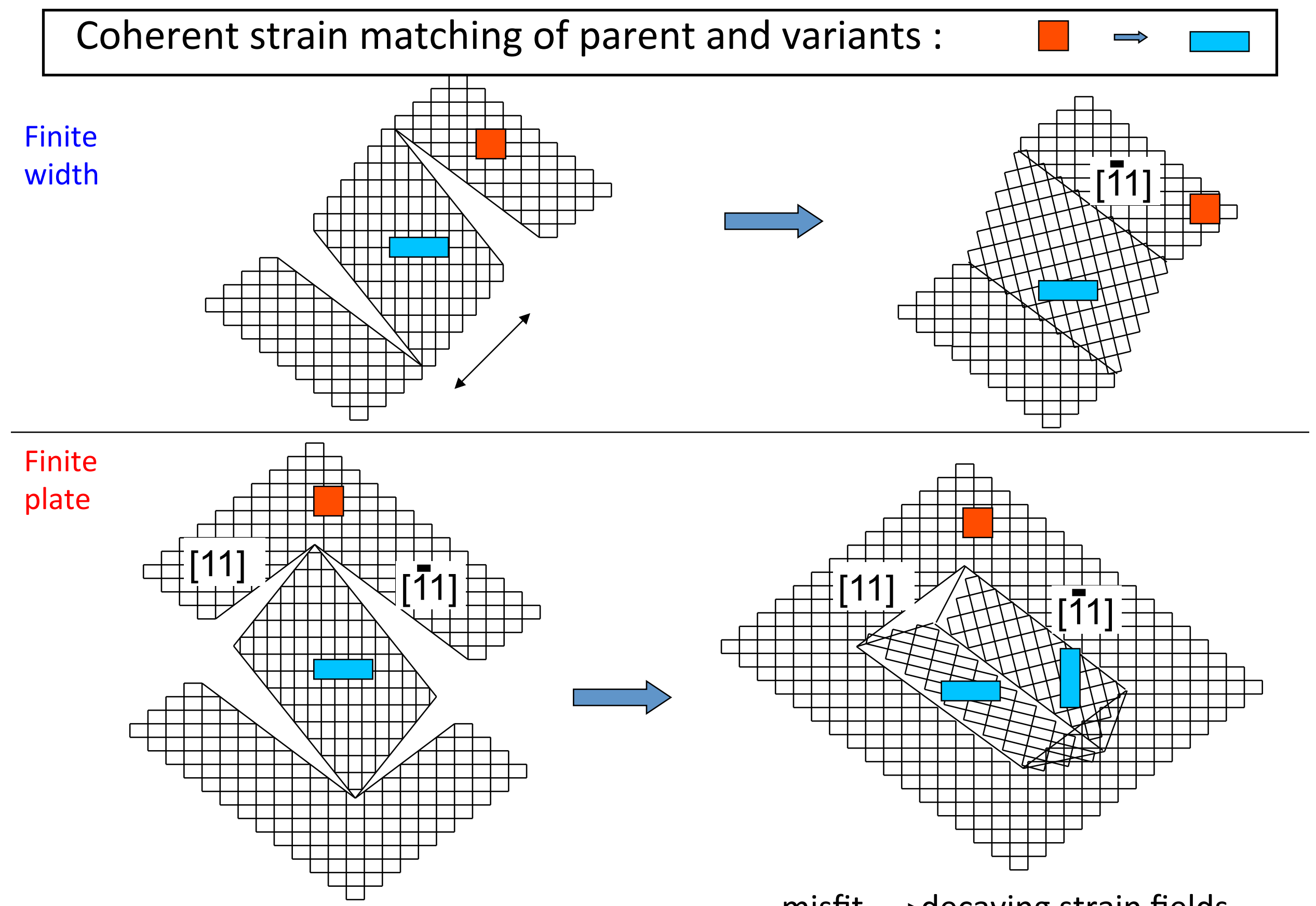

M. Porta et al., 2010

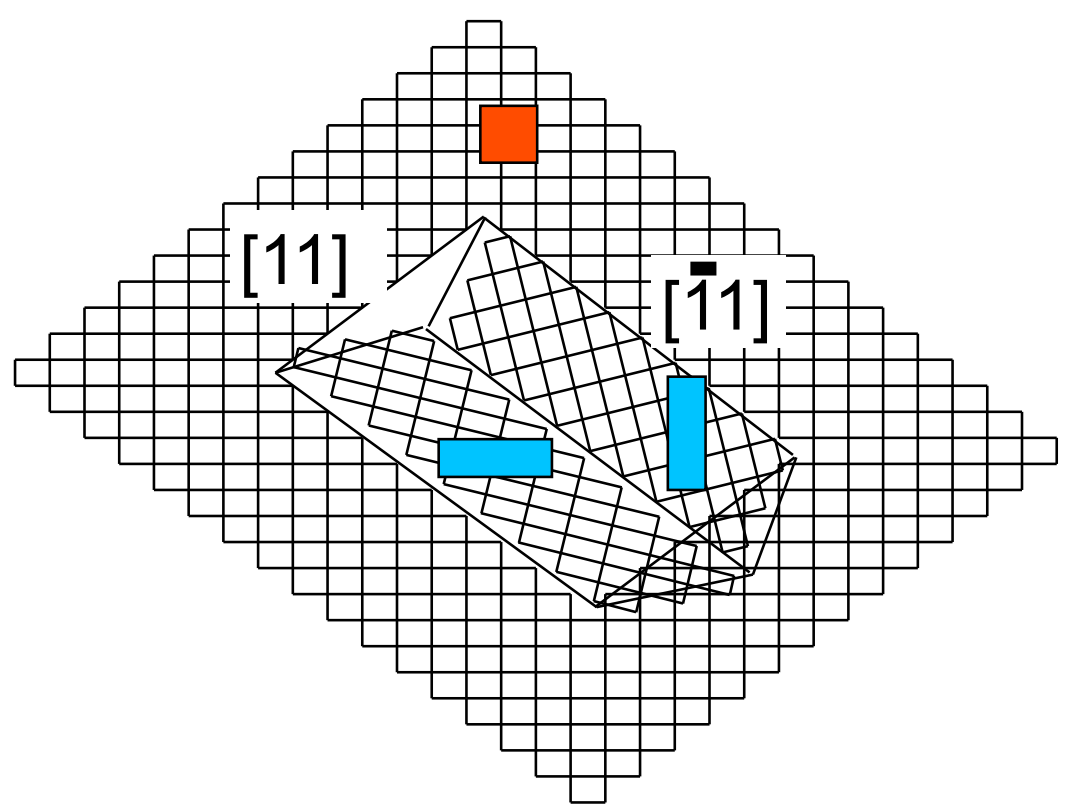

misfit $\longrightarrow$ decaying strain fields 


\section{Strains \& Compatibility}

$\varepsilon_{i j}=\frac{1}{2}\left(\frac{\partial u_{i}}{\partial x_{j}}+\frac{\partial u_{j}}{\partial x_{i}}\right)$
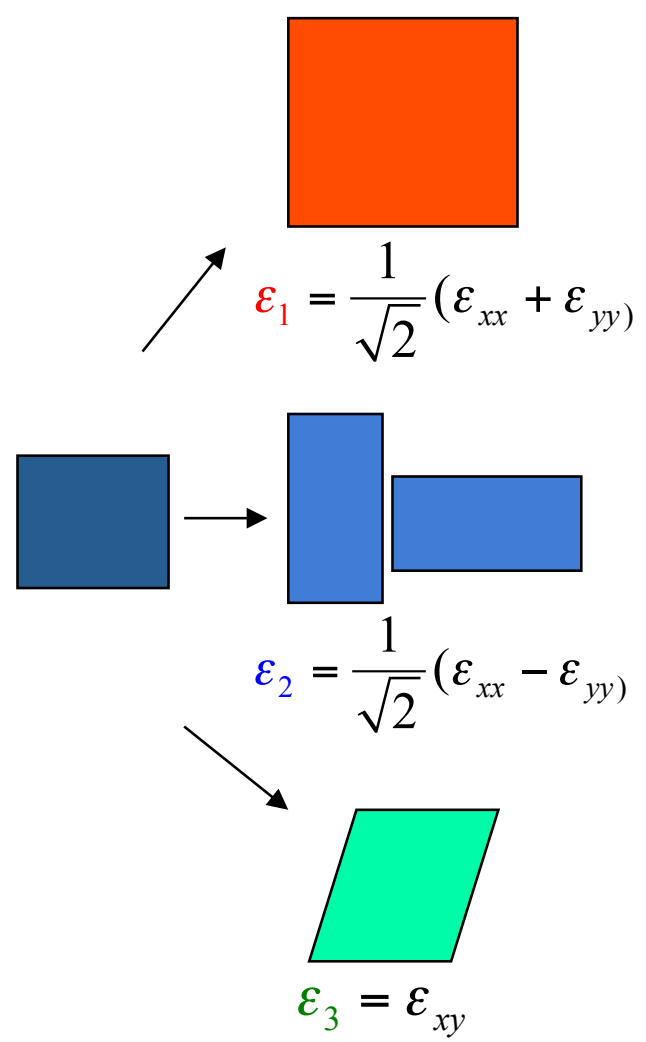

Elastic energy:

$$
=A_{1} \varepsilon_{1}{ }^{2}+A_{2} \varepsilon_{2}{ }^{2}+A_{3} \varepsilon_{3}{ }^{2}
$$

\section{Elastic compatibility:}

$$
\nabla^{2} \varepsilon_{1}-\left(\frac{\partial^{2}}{\partial x^{2}}-\frac{\partial^{2}}{\partial y^{2}}\right) \varepsilon_{2}-\sqrt{8} \frac{\partial^{2}}{\partial x \partial y} \varepsilon_{3}=0
$$

(a) coherent

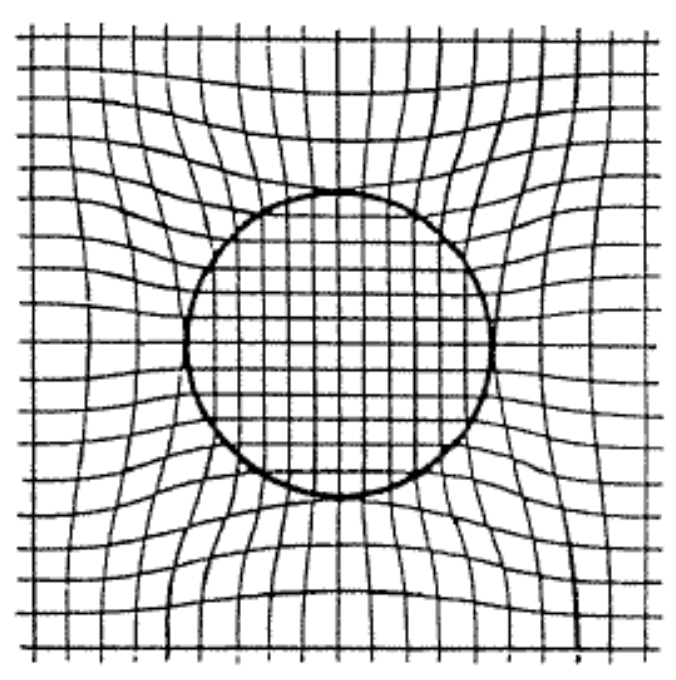

(b) incoherent

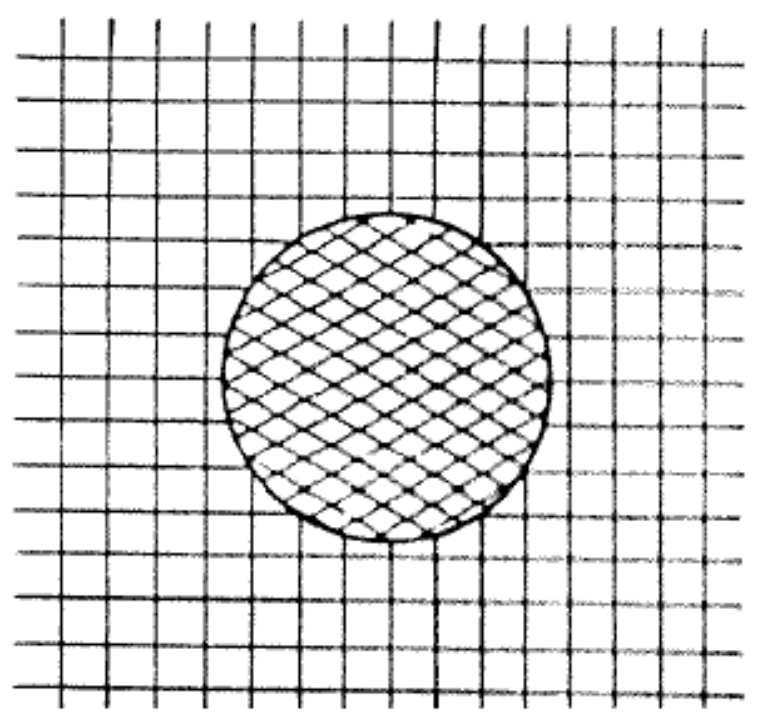




\section{Free energy for $\square \rightarrow \square$}

$$
F=\int d \vec{r}\left[F_{\text {elas }}+F_{\text {struct }}+F_{\text {load }}\right]
$$

$$
F_{\text {struct }}\left(\varepsilon_{2}\right)=\frac{a\left(T-T_{0}\right)}{2} \varepsilon_{2}{ }^{2}-\frac{B}{4} \varepsilon_{2}{ }^{4}+\frac{C}{6} \varepsilon_{2}{ }^{6}+\frac{g}{2}\left|\nabla \varepsilon_{2}\right|^{2}
$$

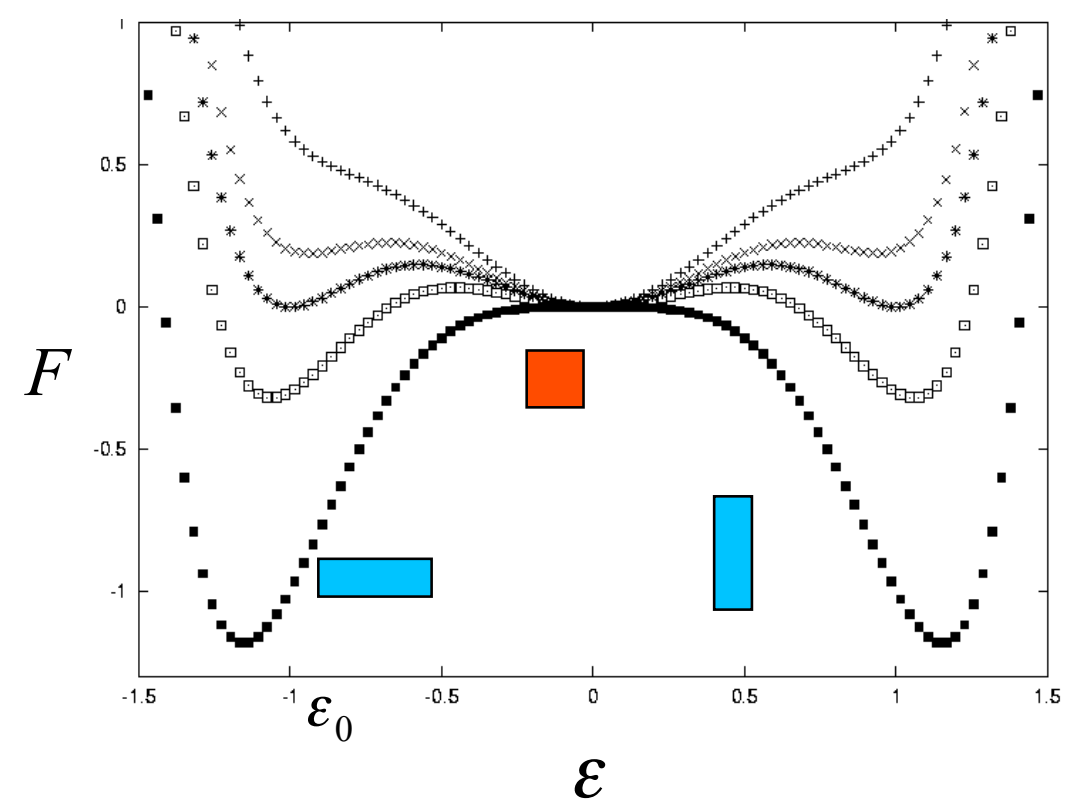




$$
F=\int d \vec{r}\left[F_{\text {elas }}+F_{\text {struct }}+F_{\text {load }}\right]
$$

$$
F_{\text {struct }}\left(\varepsilon_{2}\right)=\frac{a\left(T-T_{0}\right)}{2} \varepsilon_{2}{ }^{2}-\frac{B}{4} \varepsilon_{2}{ }^{4}+\frac{C}{6} \varepsilon_{2}{ }^{6}+\frac{g}{2}\left|\nabla \varepsilon_{2}\right|^{2}
$$

Uniaxial External Stress:

$$
F_{\text {load }}=-\sigma \varepsilon_{x x}=-\frac{\sigma}{\sqrt{2}}\left(\varepsilon_{1}+\varepsilon_{2}\right)
$$

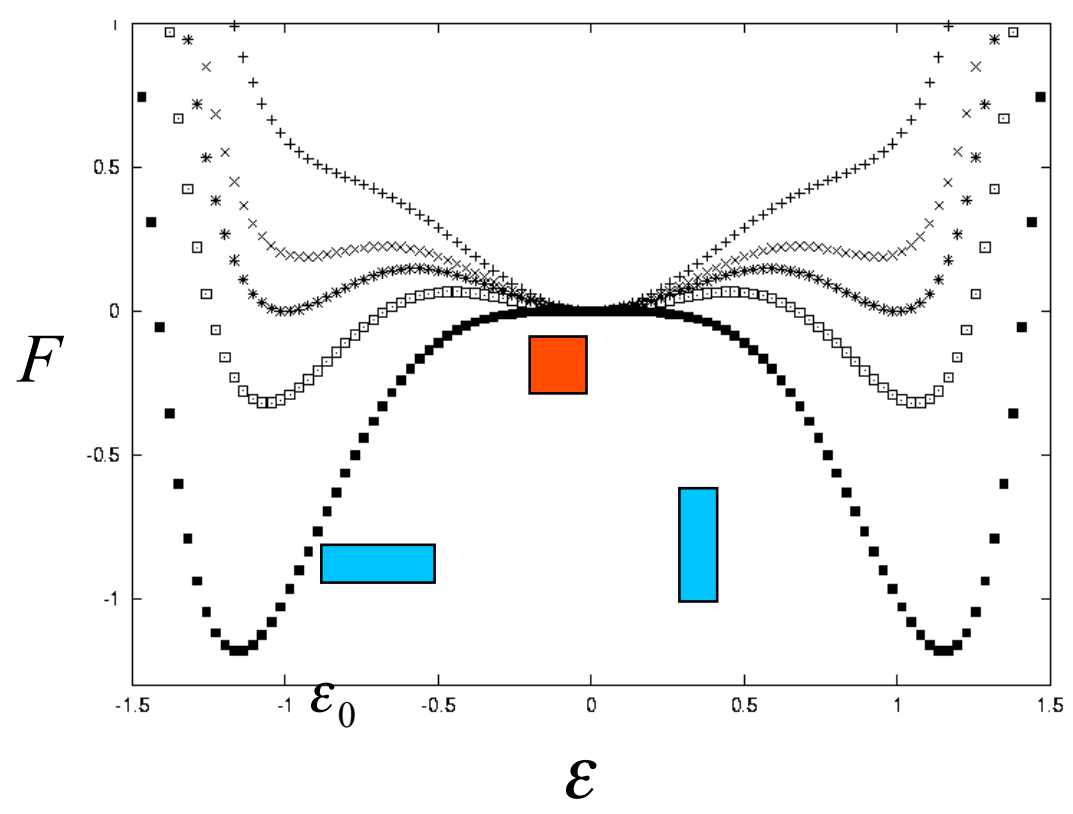




\section{ANISOTROPIC LONG-RANGE INTERACTION}

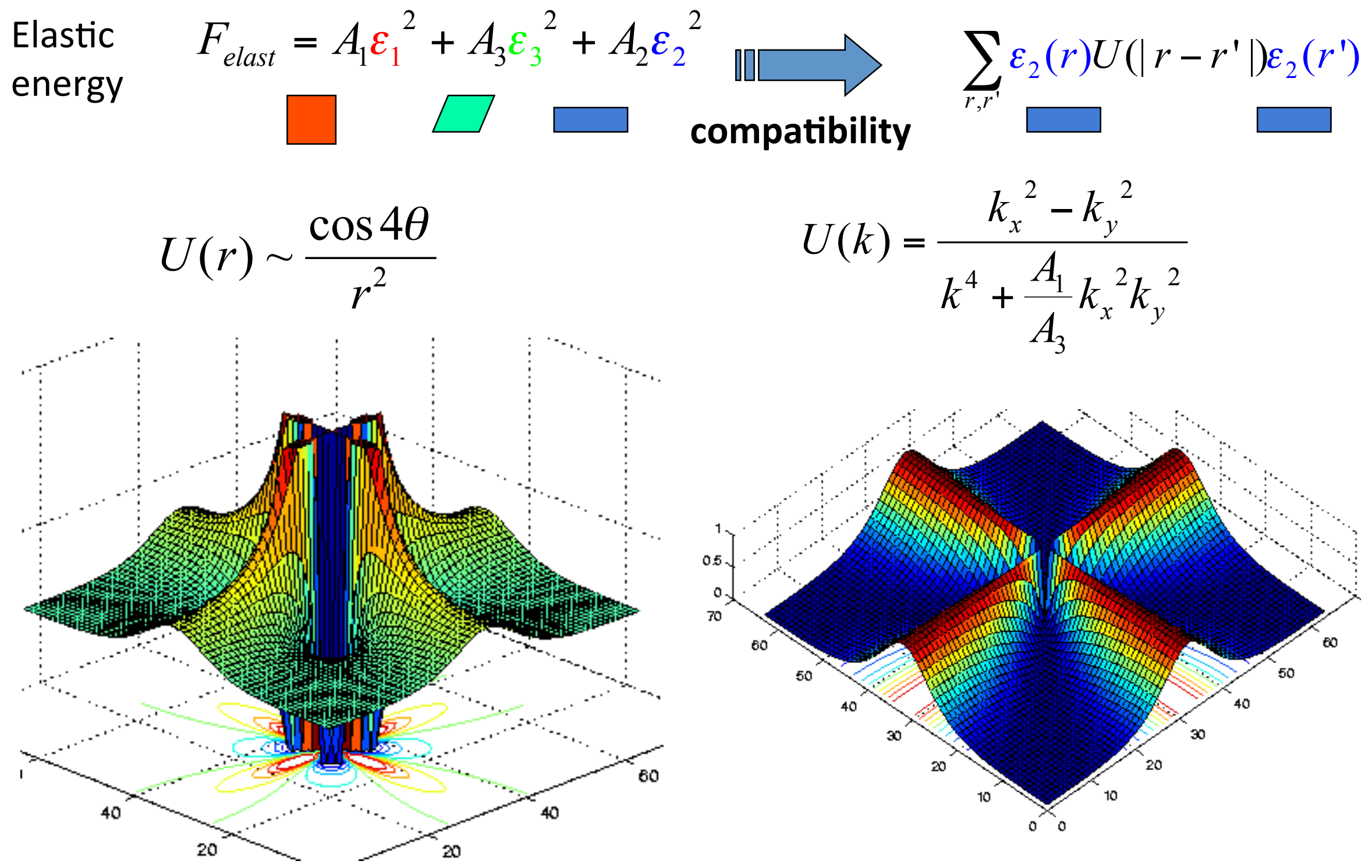

Shenoy et .al. PRB (1999), Lookman et al. PRB (2003) 


\section{Defect induced microstructure}
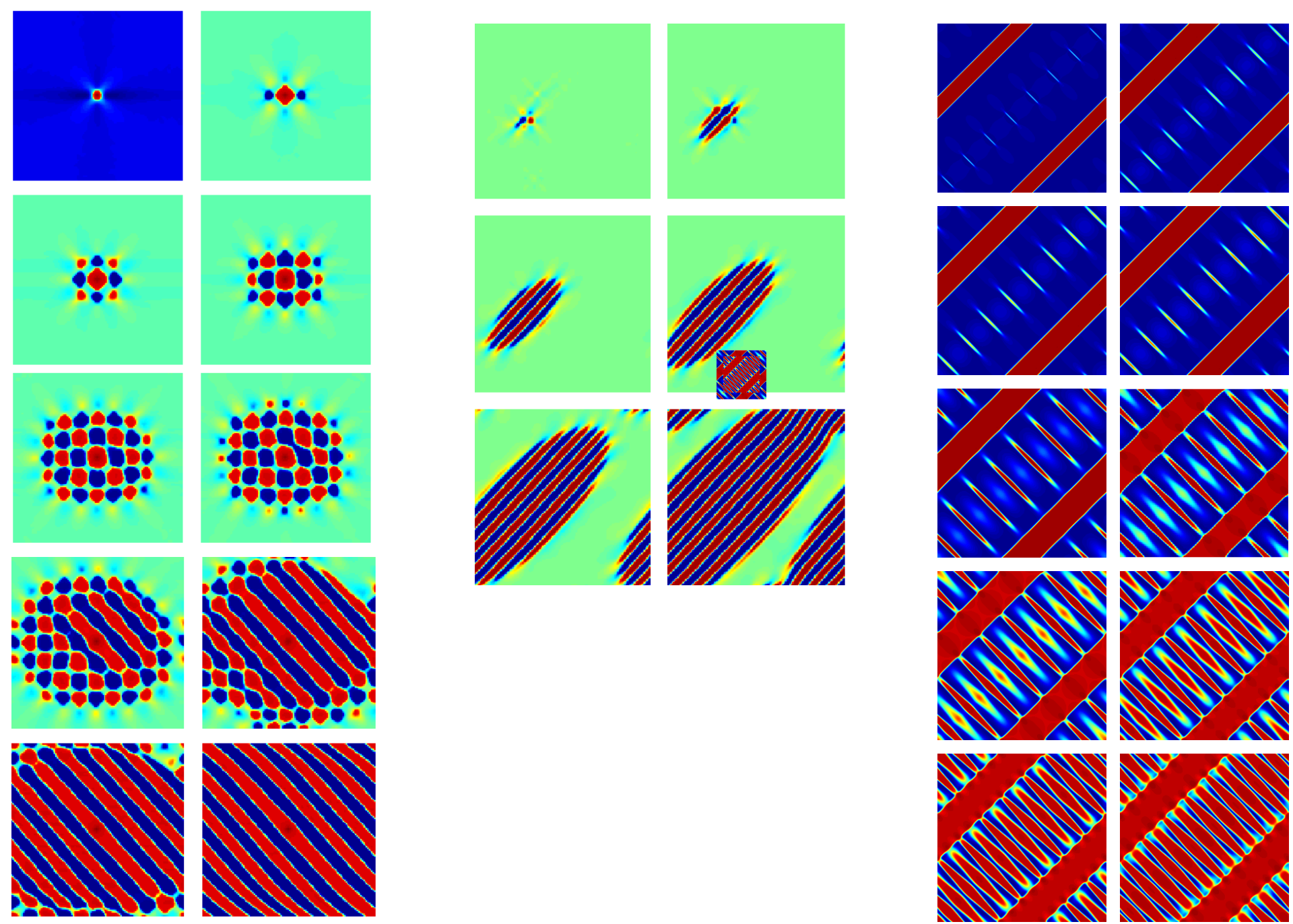

- initial state with $\square$ seeds within
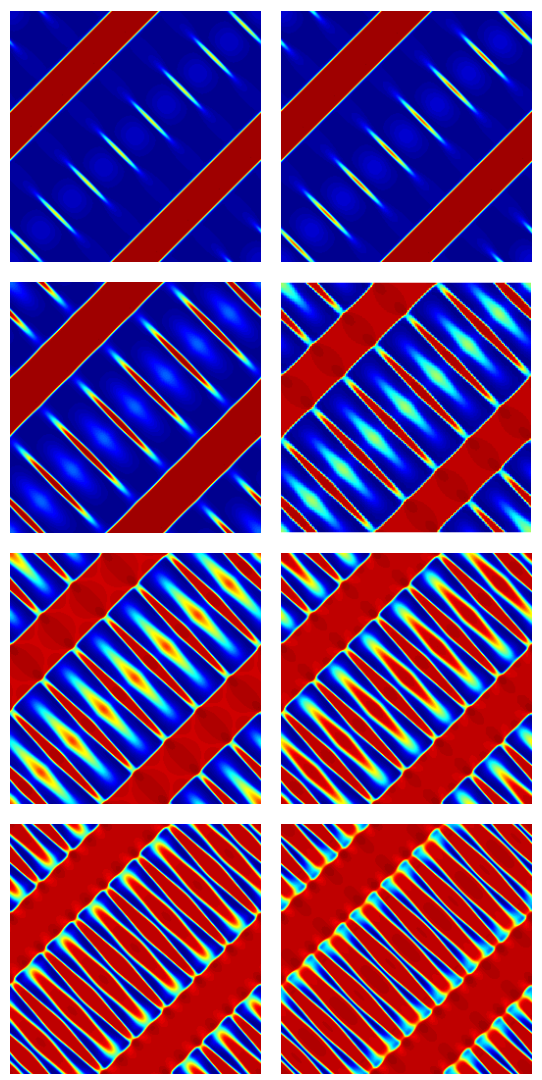

- tetragonal defect within

Lookman et al. 2003 


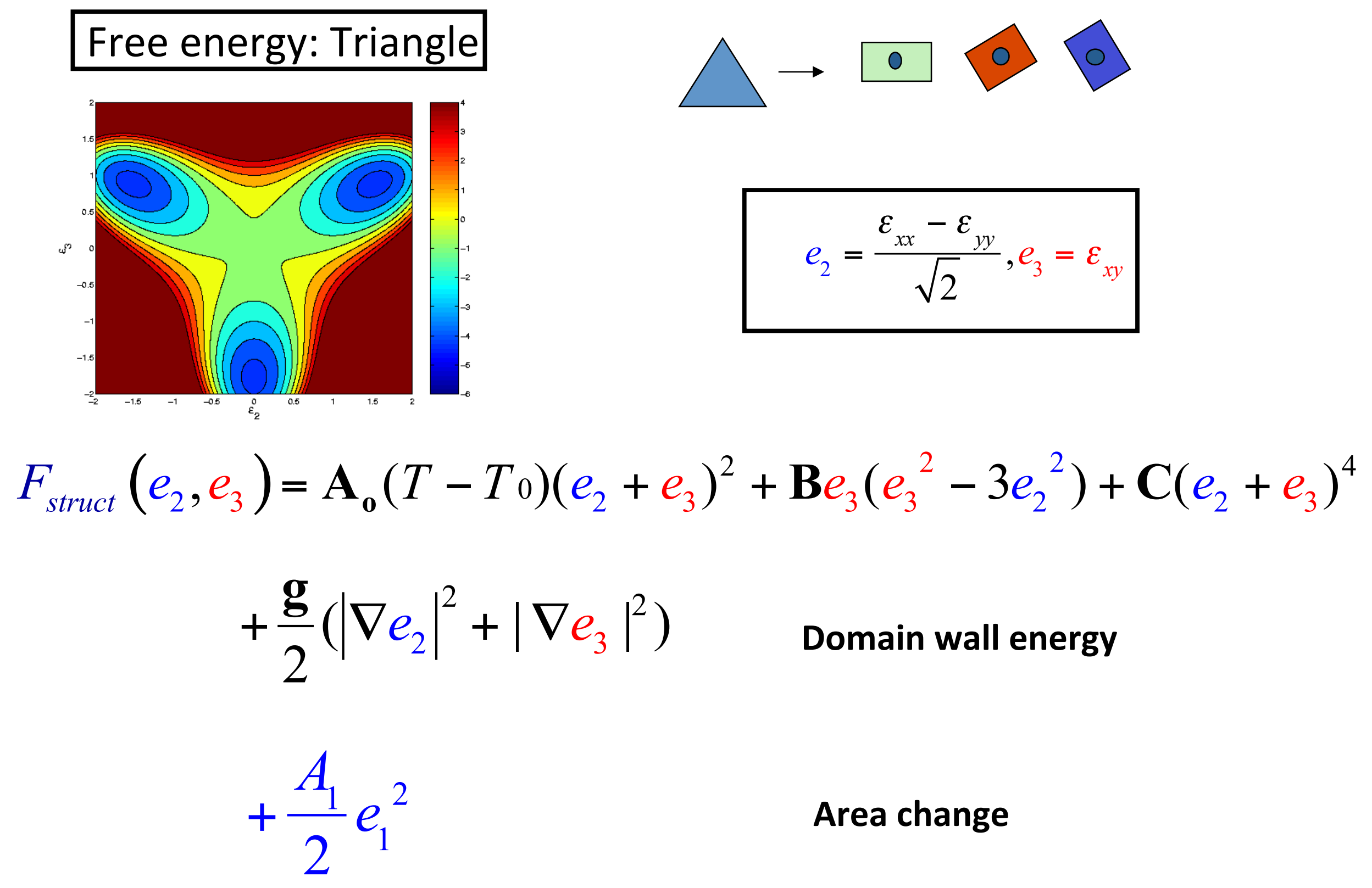




\section{Lead Orthovanadate:}

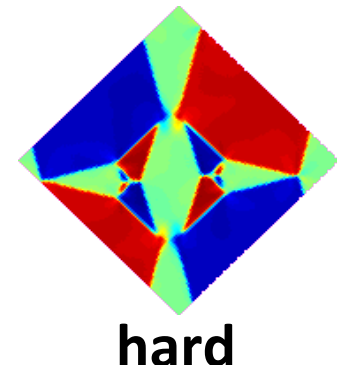

Underdamped
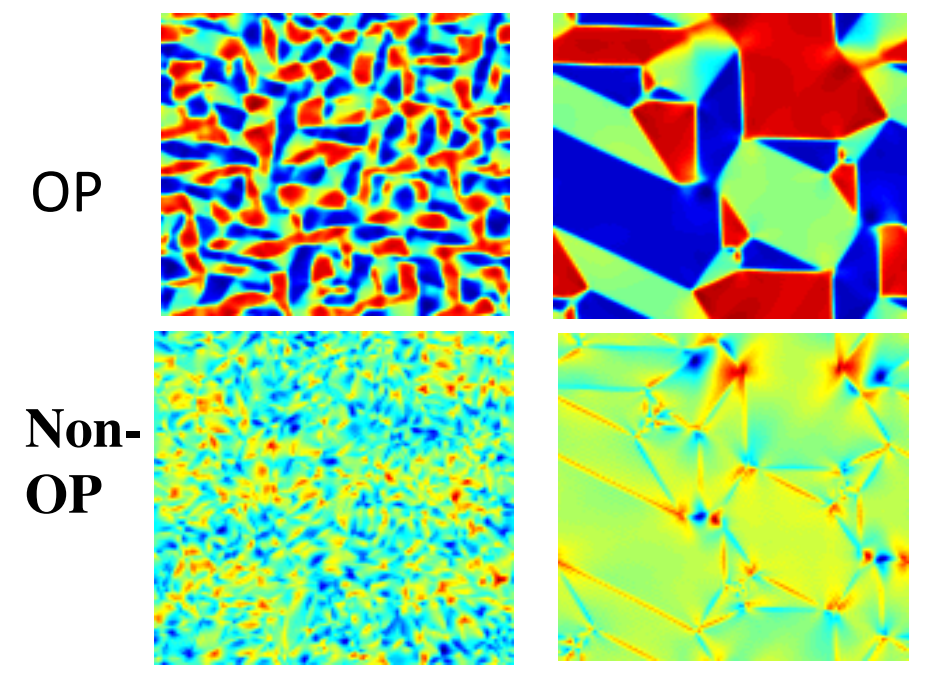

$\mathrm{Pb}\left(\mathrm{VO}_{4}\right)_{3}$
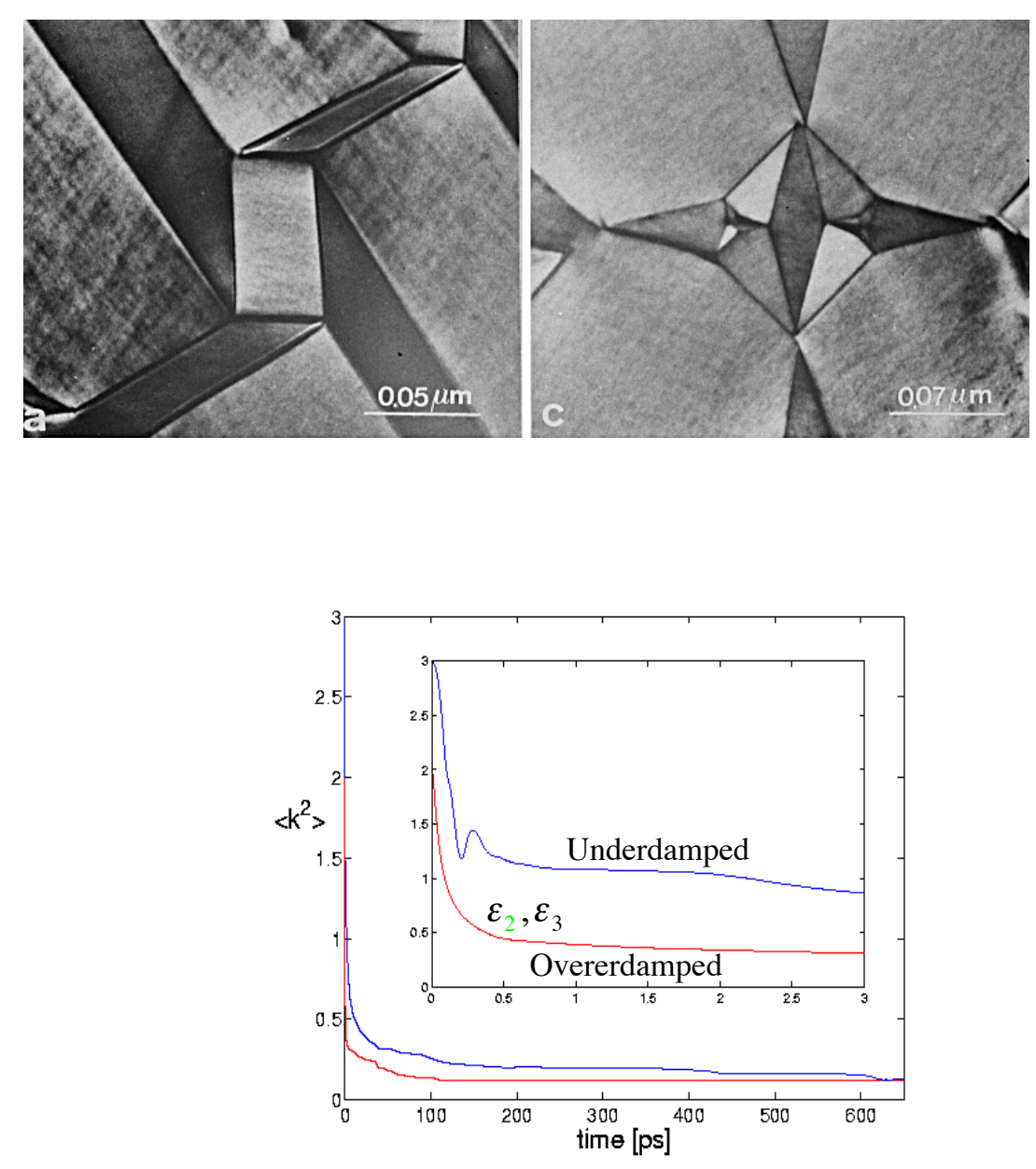

Lookman et al. Phys. Rev. B, 67, 024114 (2003) 
-Example: Cubic to Tetragonal
(I)

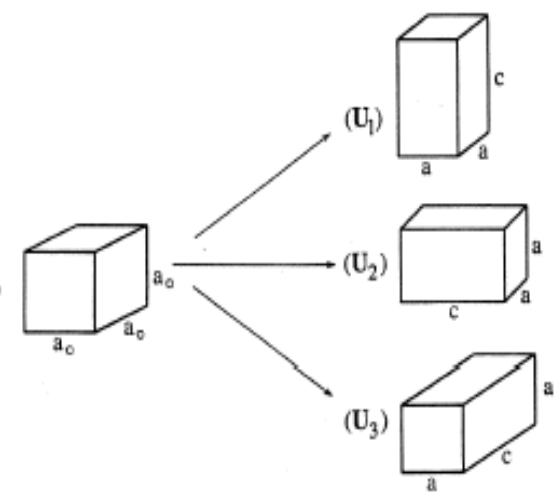

Austenite
Figure 4.4: The three variants of martensite in a cubic to tetragonal transformation.

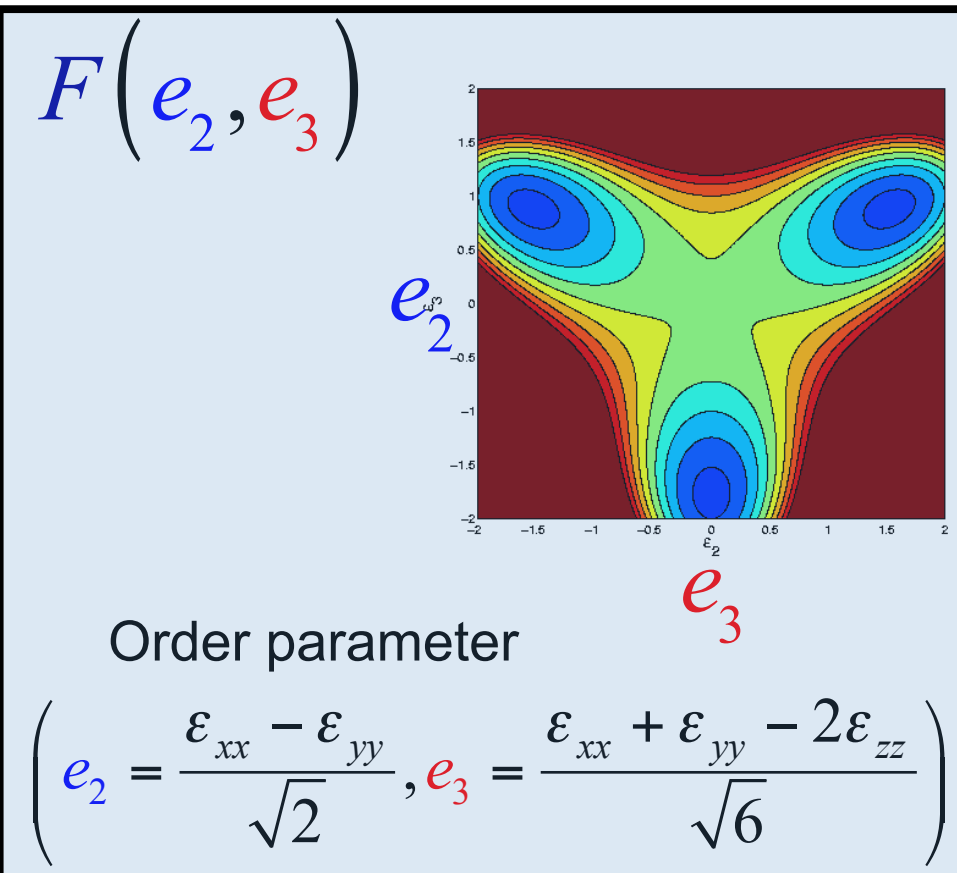

Barsch \&Krumhansl, 84, Falk, 83

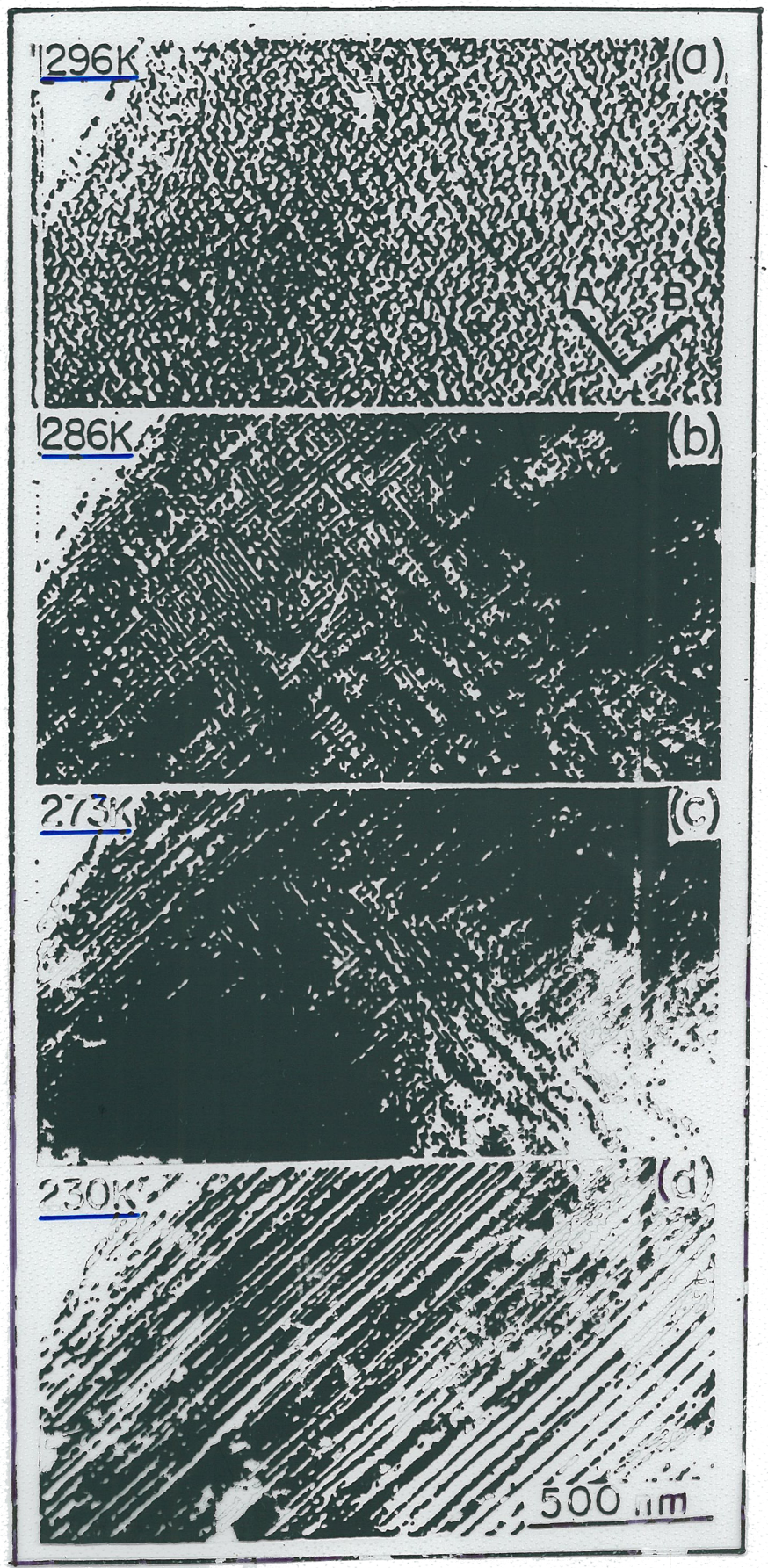

$\mathrm{Fe}_{0.7} \mathrm{Pd}_{0.3}$

Austenite

TWEED

$T_{0}=268 \mathrm{~K}$

(TWINNED)

MARTENSITE

Sugiyama (1985) 


\section{SYMMETRY ADAPTED STRAINS FOR CUBIC SYSTEM}
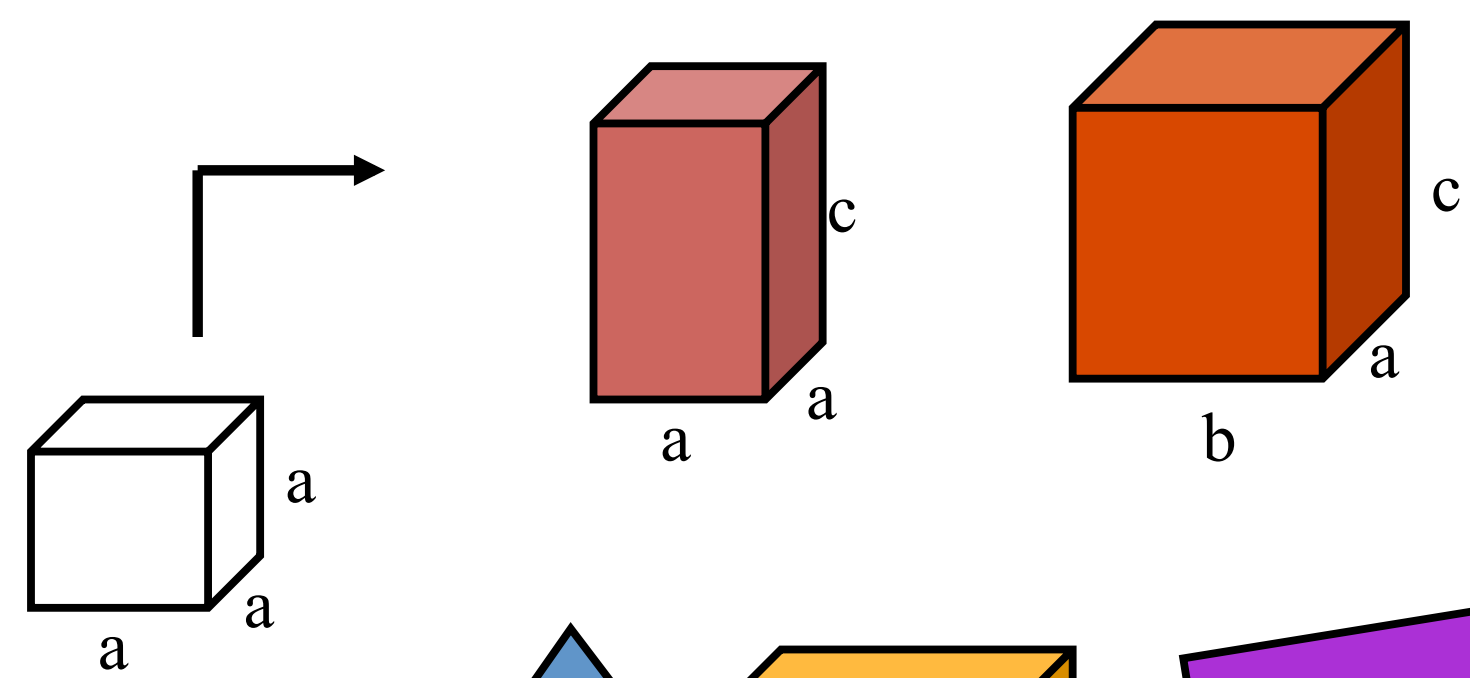

$$
\left[\begin{array}{c}
e_{2}=\varepsilon_{x x}-\varepsilon_{y y} \\
e_{3}=\varepsilon_{x x}+\varepsilon_{y y}-2 \varepsilon_{z z}
\end{array}\right]
$$
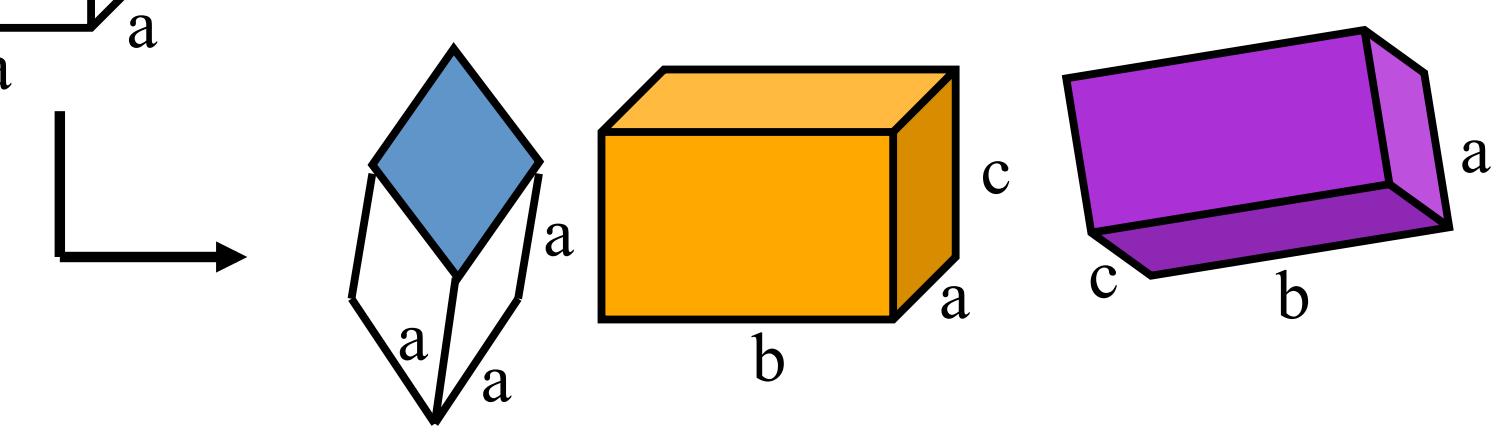

$$
\left[\begin{array}{l}
e_{4}=\varepsilon_{x y} \\
e_{5}=\varepsilon_{x z} \\
e_{6}=\varepsilon_{y z}
\end{array}\right]
$$

$$
\begin{aligned}
& e_{1}=\varepsilon_{x x}+\varepsilon_{y y}+\varepsilon_{z z} \quad e_{1} \quad e_{2}, e_{3} \quad e_{4}, e_{5}, e_{6} \\
& 3[111] \quad e_{1}-\frac{1}{2} e_{2}+\frac{\sqrt{3}}{2} e_{3},-\frac{\sqrt{3}}{2} e_{2}-\frac{1}{2} e_{3} \quad e_{6}, e_{4}, e_{5} \\
& 4[001] \quad e_{1} \\
& -e_{2}, e_{3} \\
& -e_{4}, e_{6},-e_{5} \\
& 2[110] \\
& -e_{2}, e_{3} \\
& e_{4},-e_{6},-e_{5}
\end{aligned}
$$




\section{Free energy for Cubic}

$\Longrightarrow$ Tetragonal transition

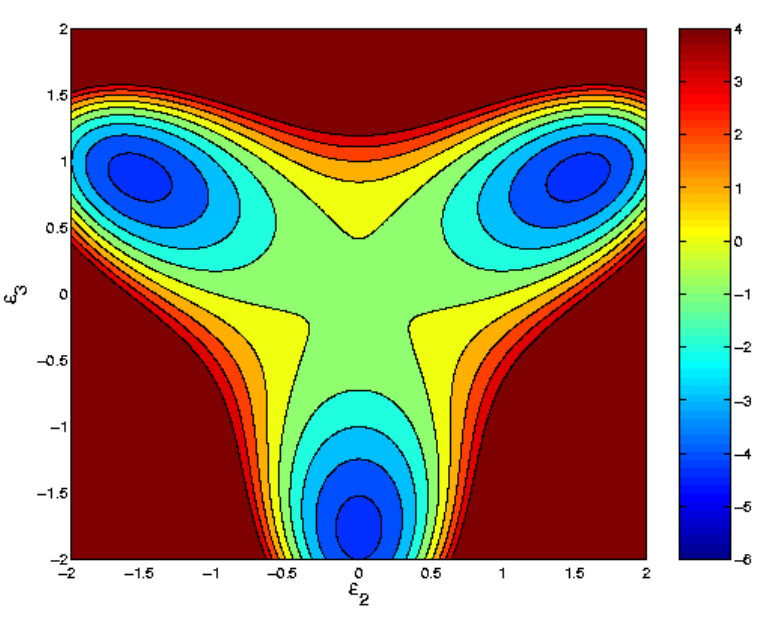

Order Parameter

$$
\begin{aligned}
& F\left(e_{2}, e_{3}\right)=\mathbf{A}_{\mathbf{o}}(\left.T-T_{0}\right)\left(e_{2}^{2}+e_{3}^{2}\right)+\mathbf{B} e_{3}\left(e_{3}^{2}-3 e_{2}^{2}\right)+\mathbf{C}\left(e_{2}^{2}+e_{3}^{2}\right)^{2} \\
&+\mathbf{E} e_{1}\left(e_{2}^{2}+e_{3}^{2}\right) \text { Transformation } \\
& \text { Volume change } \\
&+ \frac{A_{B}}{2} e_{1}^{2}+\frac{A_{S}}{2}\left(\varepsilon_{x y}^{2}+\varepsilon_{x z}^{2}+\varepsilon_{y z}^{2}\right) \leftarrow \text { Compression-shear } \\
&+\frac{\mathbf{g}}{2}\left(\left|\nabla e_{2}\right|^{2}+\left|\nabla e_{3}\right|^{2}\right) \text { Nonlocal strain } \\
& \text { inhomogeneity }
\end{aligned}
$$


Simulated 3D microstructure for a model Cubic-Tetragonal Transformation

(I)

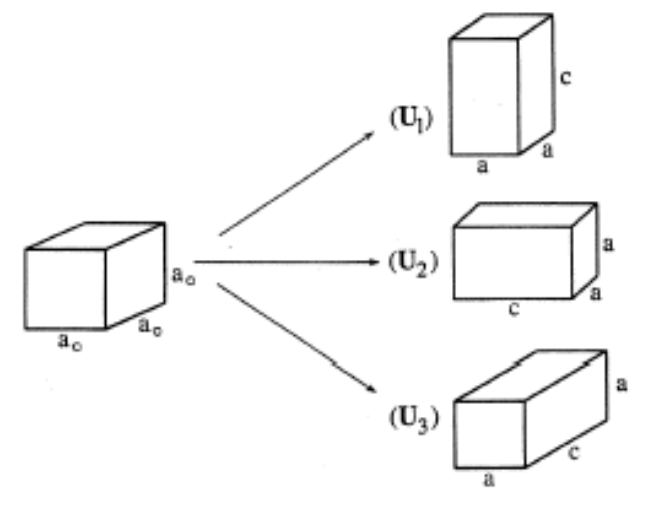

Austenite

Martensite

Figure 4.4: The three variants of martensite in a cubic to tetragonal transformation.

\section{With Volume Change}

\section{FePd}

(a)
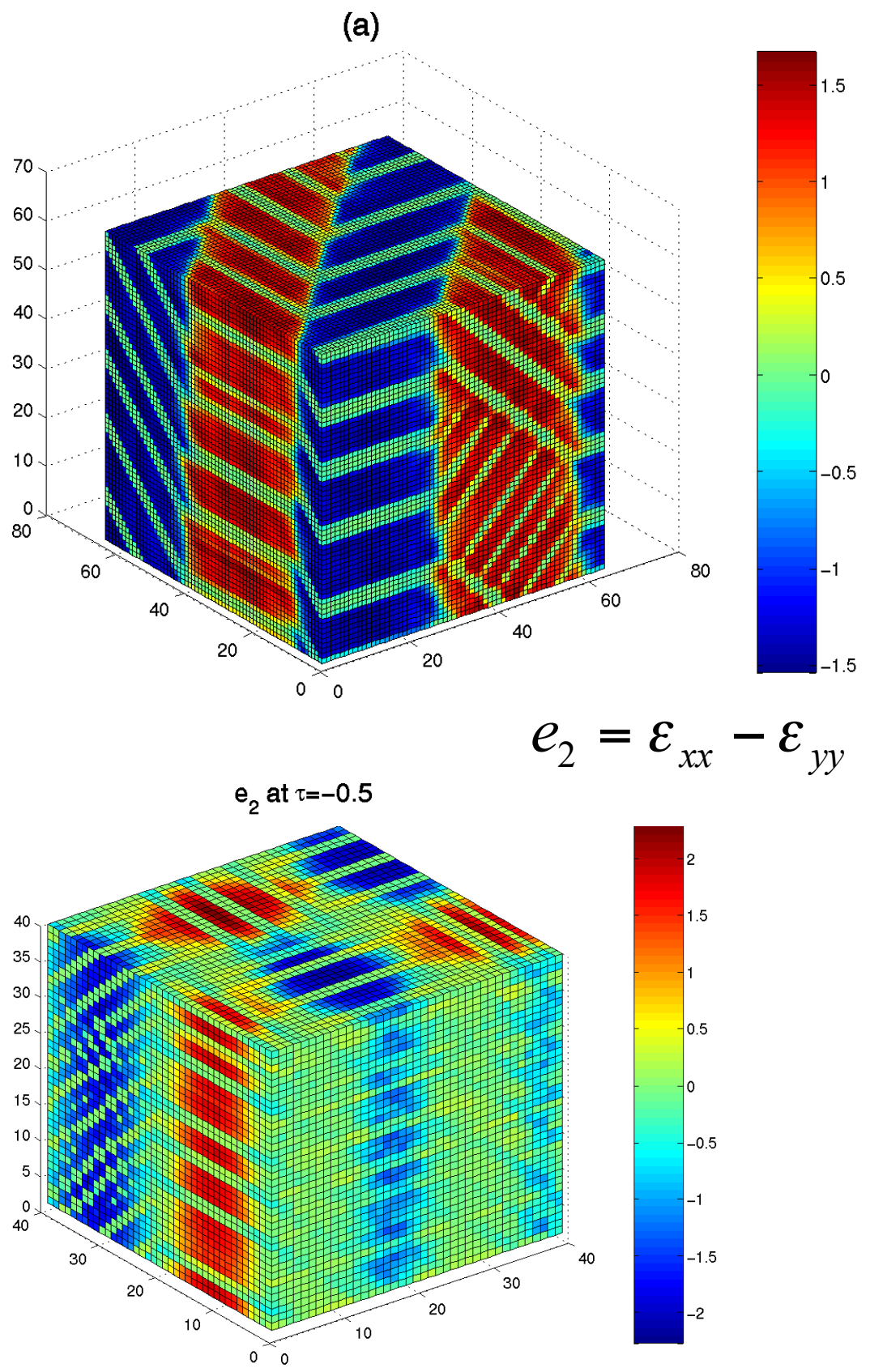


\section{DYNAMICS}




\section{EQUATION OF MOTION FOR DISPLACEMENT FIELDS}

$$
\rho \ddot{\vec{u}}=\vec{\nabla} \cdot \stackrel{\sigma}{\text { elastic }}+\vec{\nabla} \cdot \stackrel{\leftrightarrow}{\sigma}_{\text {dissipative }}
$$

$\sigma_{i j}^{\text {elastic }}=\frac{\delta F}{\delta \varepsilon_{i j}} \quad \sigma_{i j}^{\text {dissipative }}=\frac{\delta R}{\delta \dot{\varepsilon}_{i j}}$

Rayleigh Dissipation: $R=\int d \vec{r} A_{i j k l}^{\prime} \dot{\varepsilon}_{i j} \dot{\varepsilon}_{k l}$

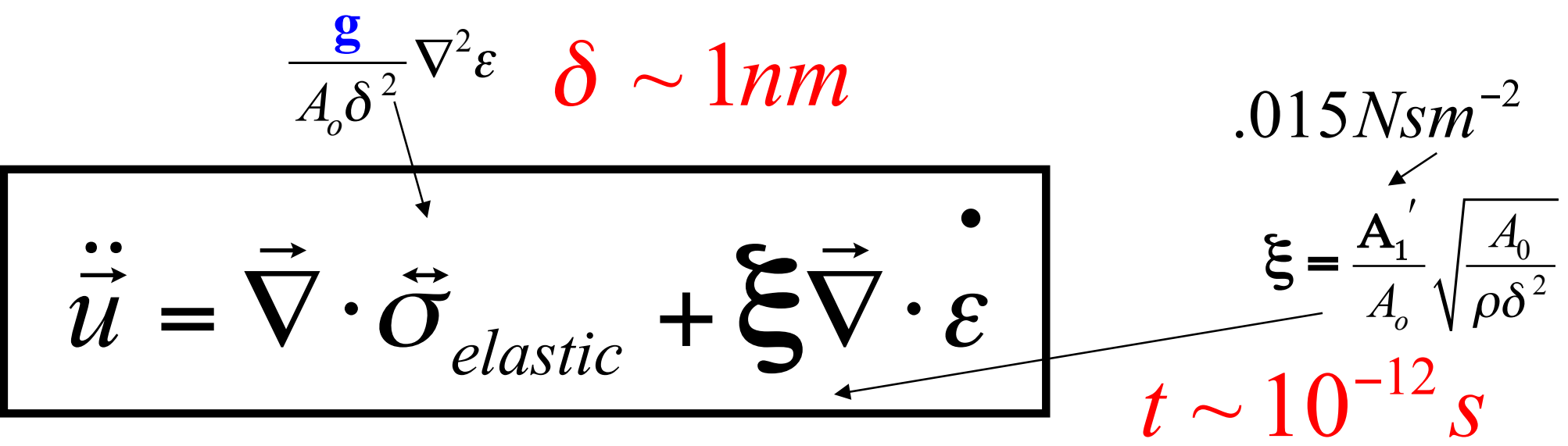




\section{STRAIN LOADING SIMULATIONS}

\section{UNIAXIAL TENSILE LOADING FOR FePd}

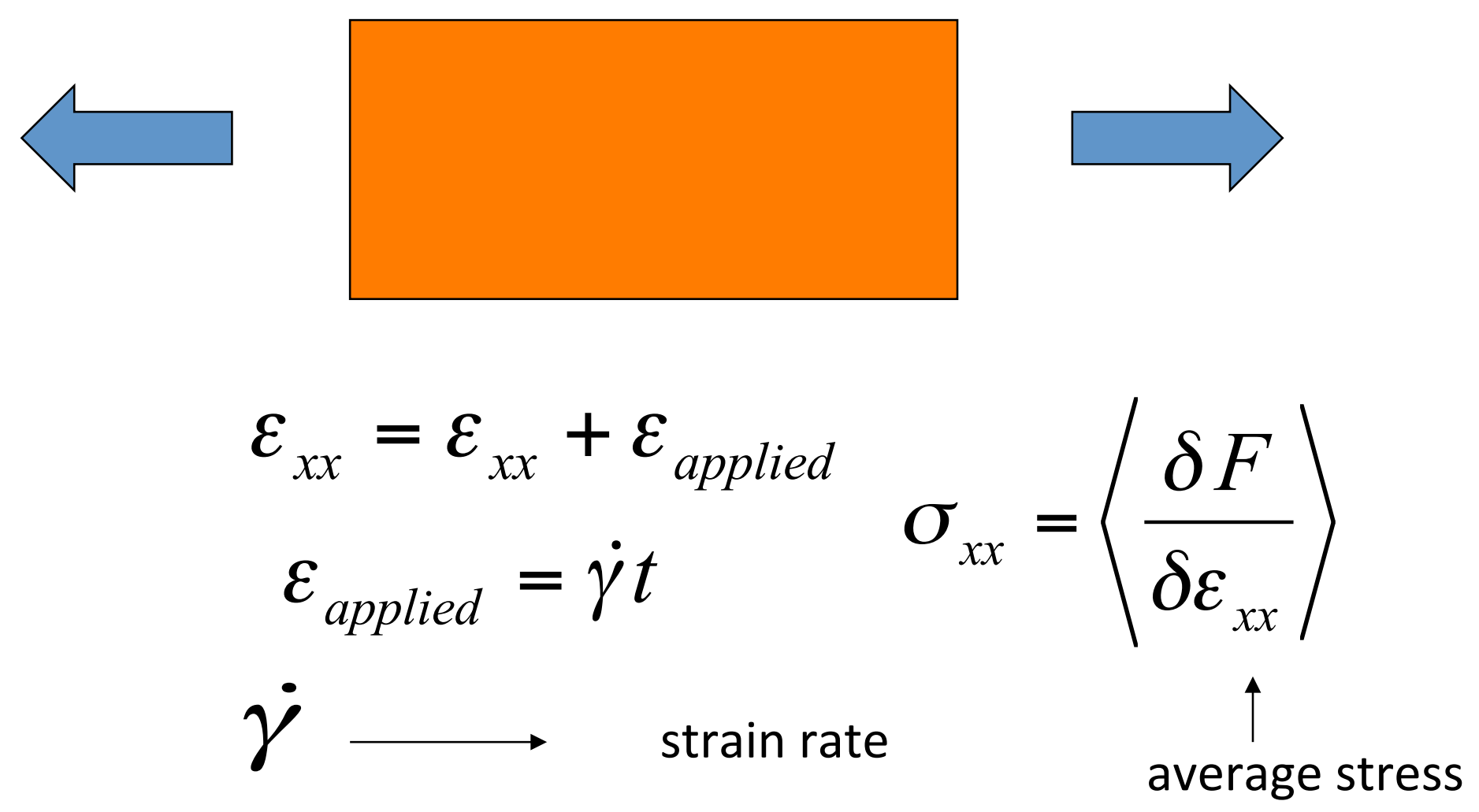

R. Ahluwalia, T. Lookman, A. Saxena, Acta Mater. 54, 2109 (2006). 


\section{MICROSTRUCTURE EVOLUTION: FePd \\ $\varepsilon_{x x}$ \\ $\varepsilon_{y y}$ \\ $\varepsilon_{z z}$}

A
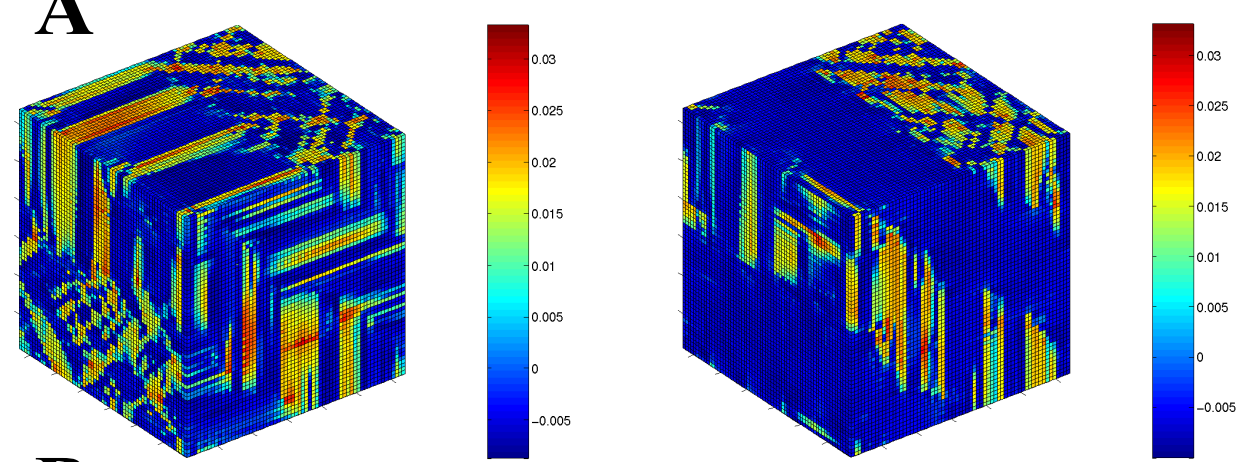

B

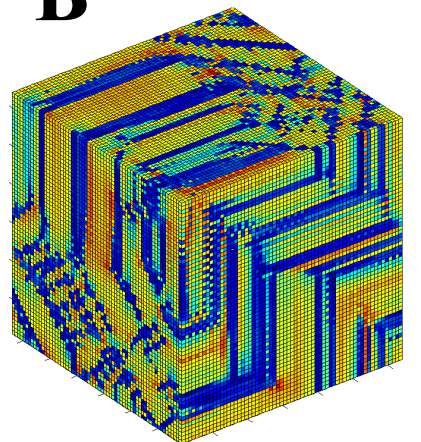

C
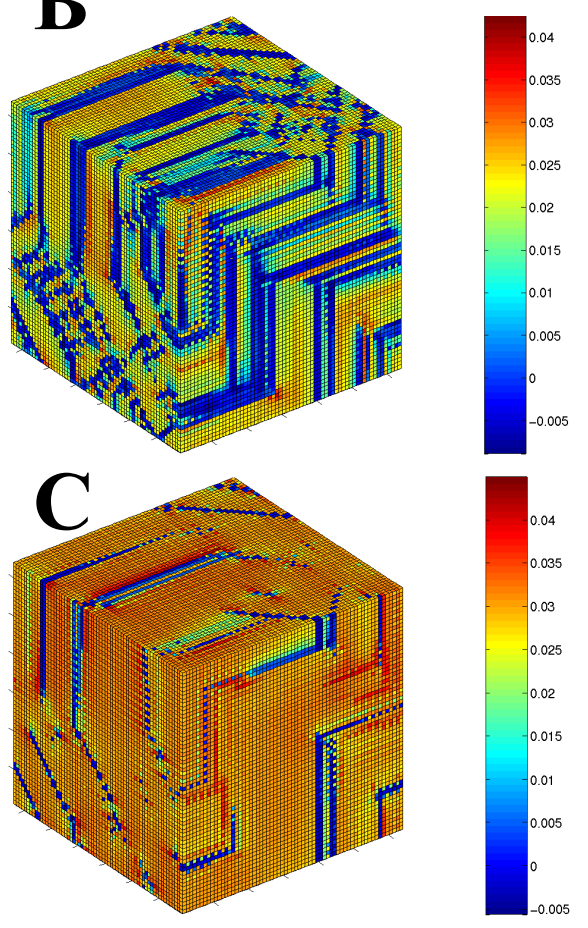
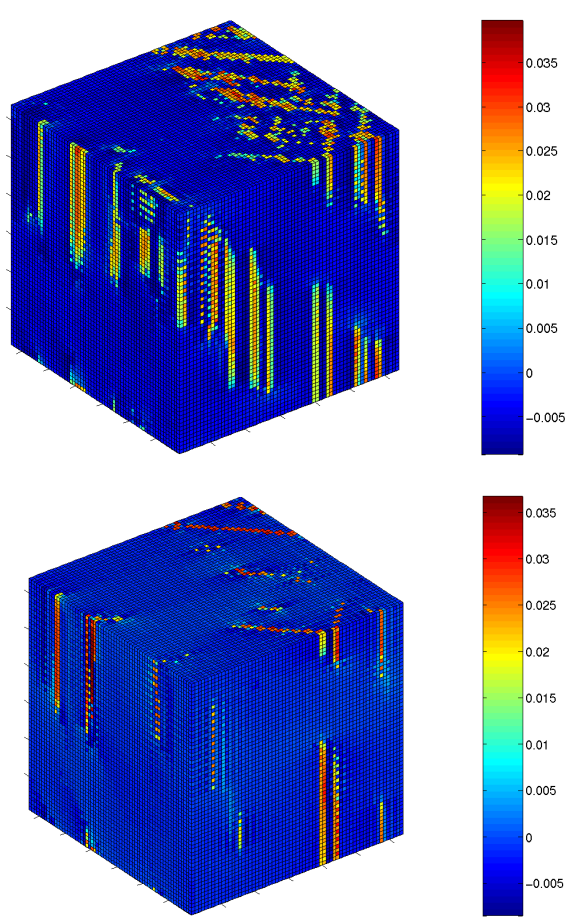
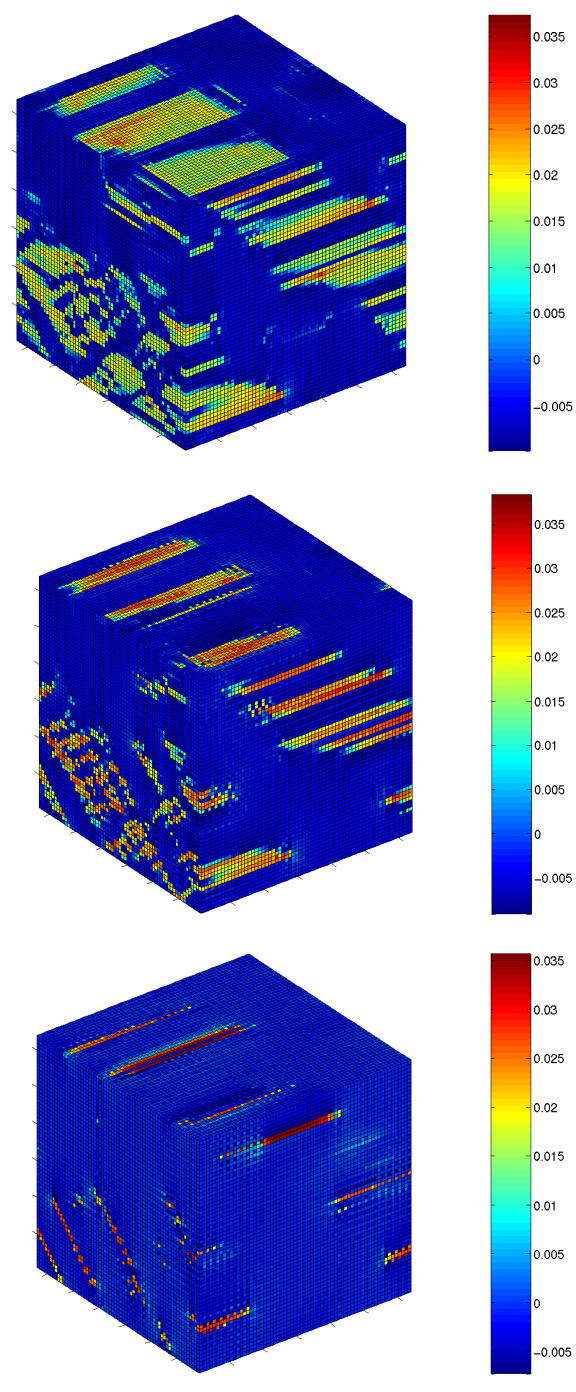


\section{STRESS-STRAIN CURVE: Uniaxial Loading}

(shape memory regime: FePd)

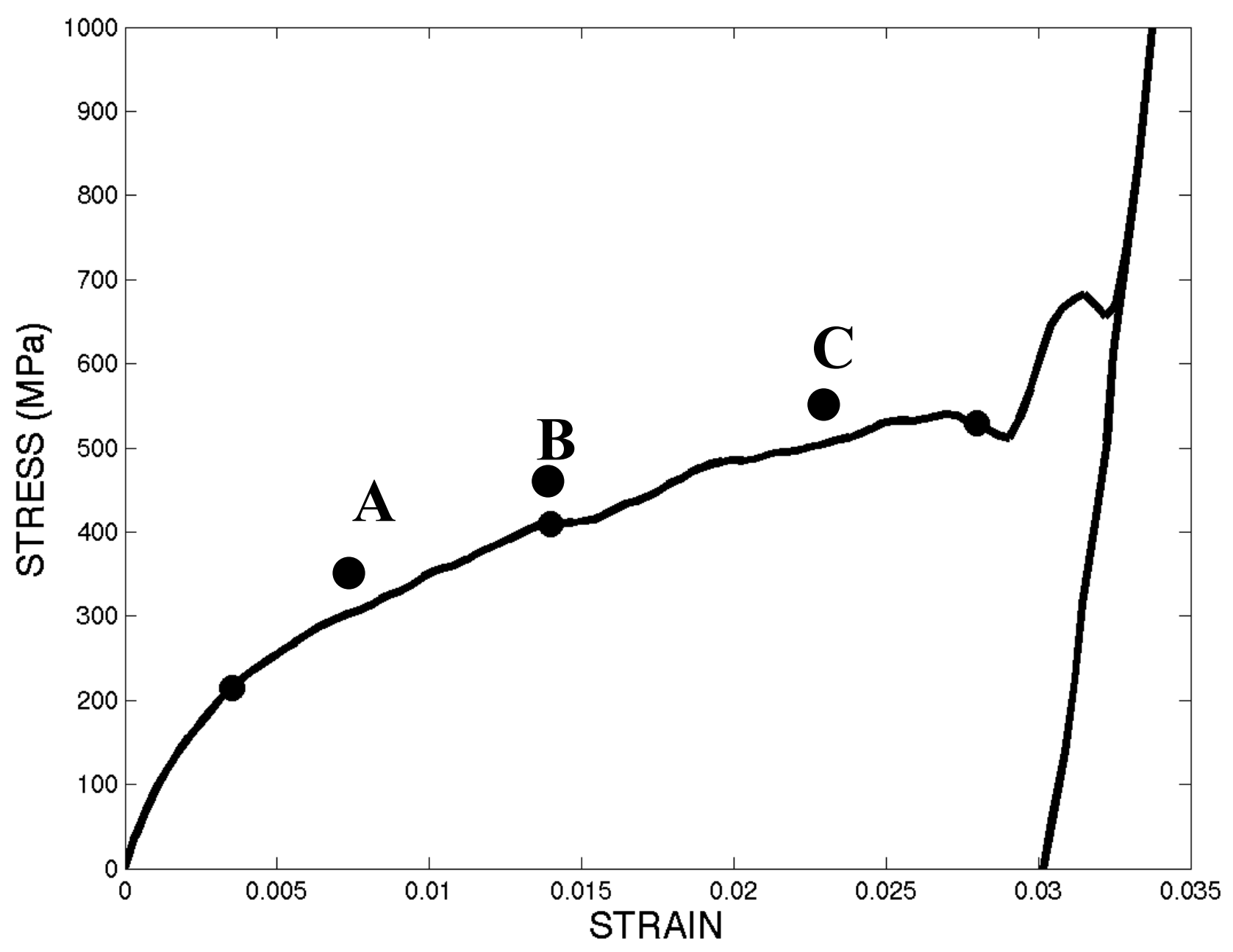




\section{STRESS-STRAIN CURVES: Three different Strain Rates}

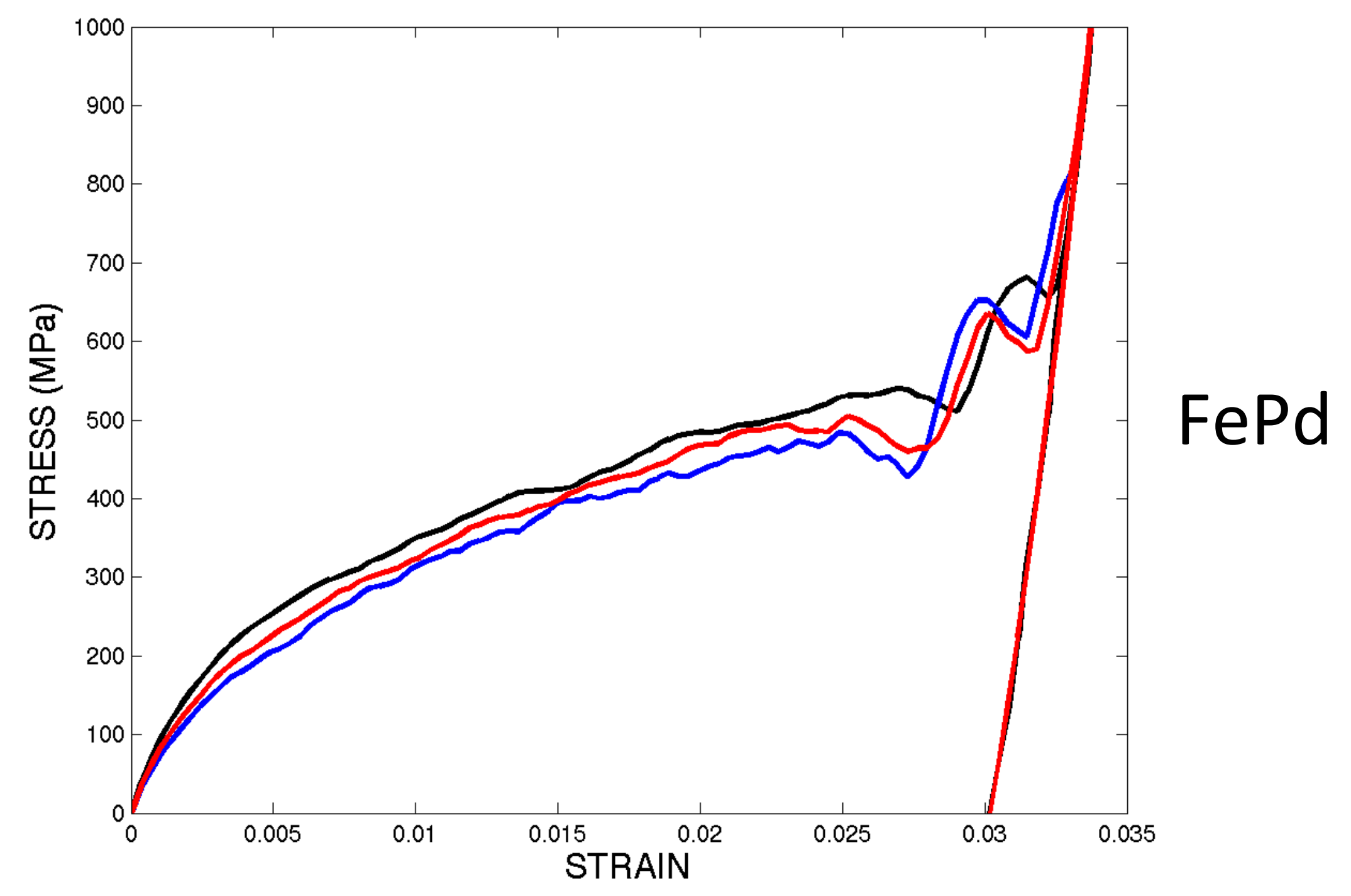


DISORDER: FERROIC GLASS 


\section{What is ergodicity?}

----- Anything that can happen will happen!

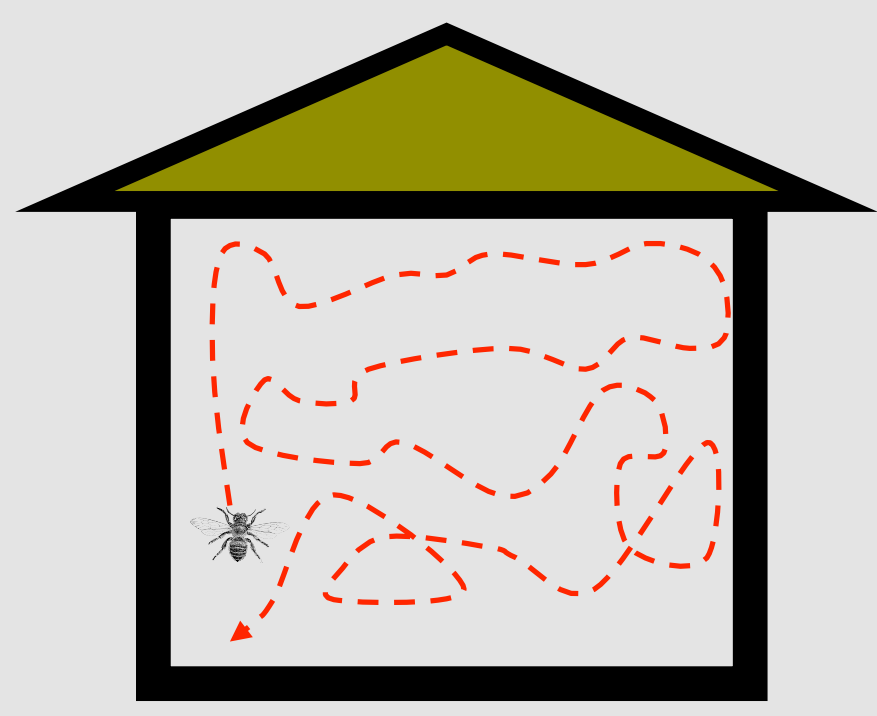

$>$ An ergodic system can visit all its possible states within experimental time.

\section{NON-ERGODICITY OF GLASS}

Short time relaxation

(Ergodicity)

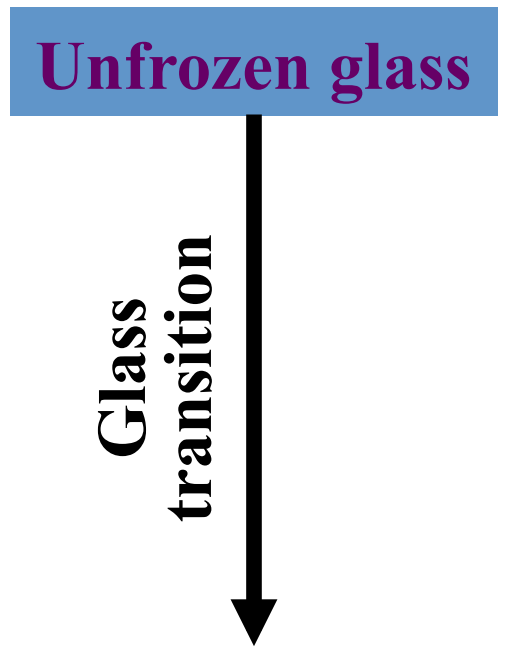

\section{Frozen glass}

Long time relaxation

(Non-ergodicity)
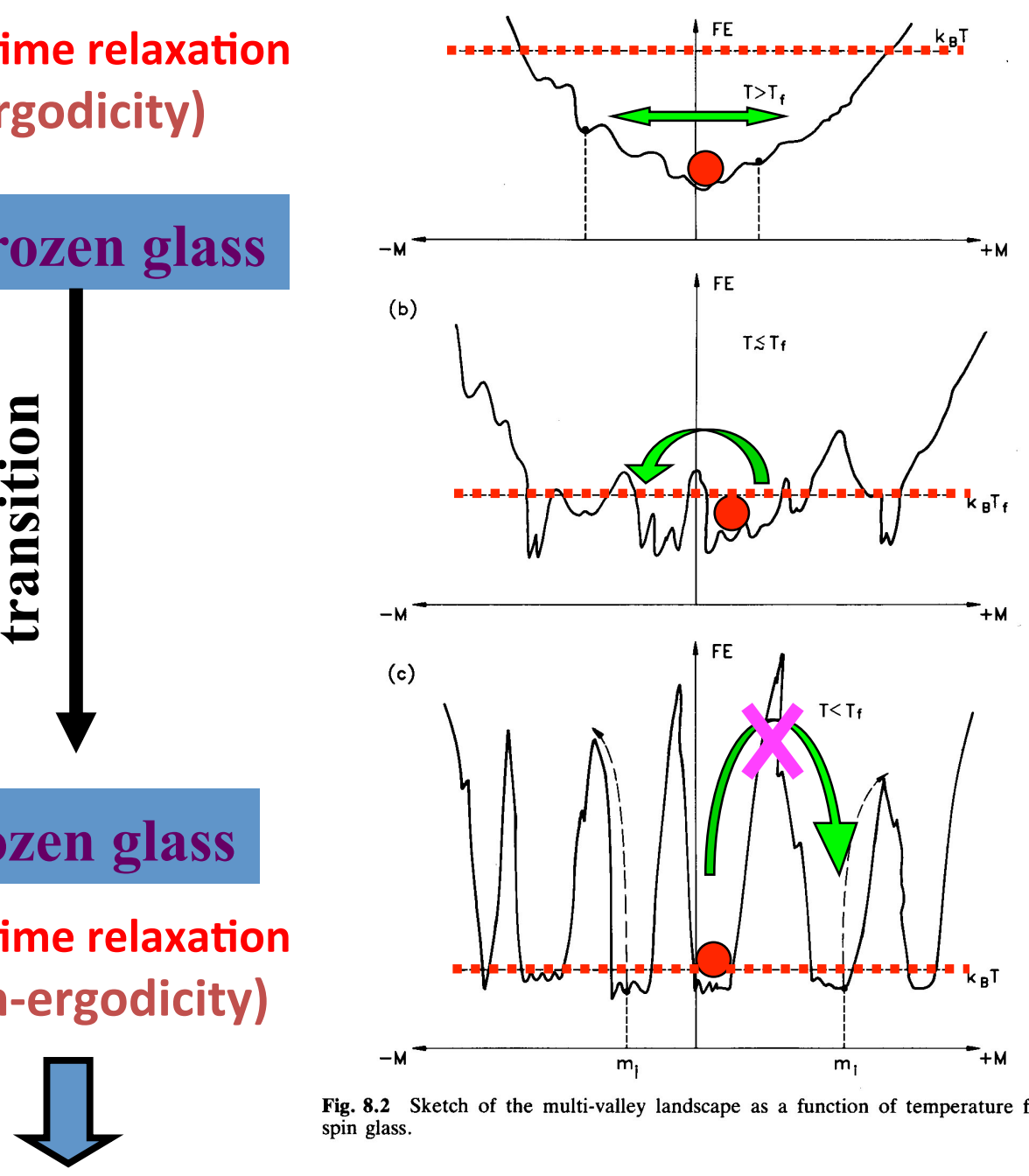

(b)

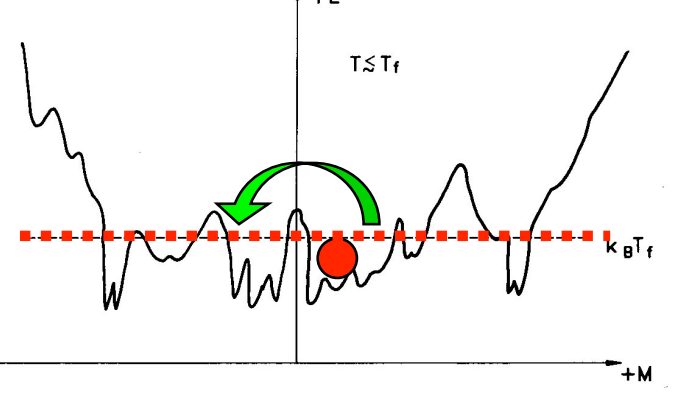

$-M$
(c)

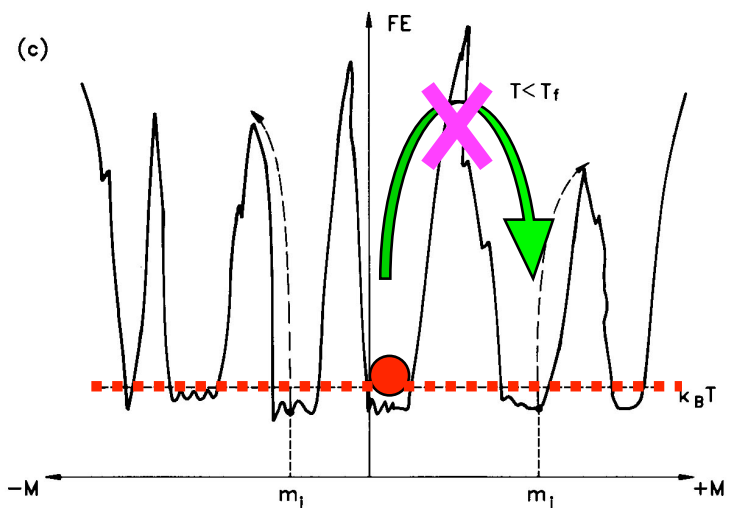

Fig. 8.2 Sketch of the multi-valley landscape as a function of temperature for a

Not all thermodynamically equivalent states are accessible in experimental time $\rightarrow$ history dependence of static properties 


\section{THREE STATES IN CONDENSED MATTER}

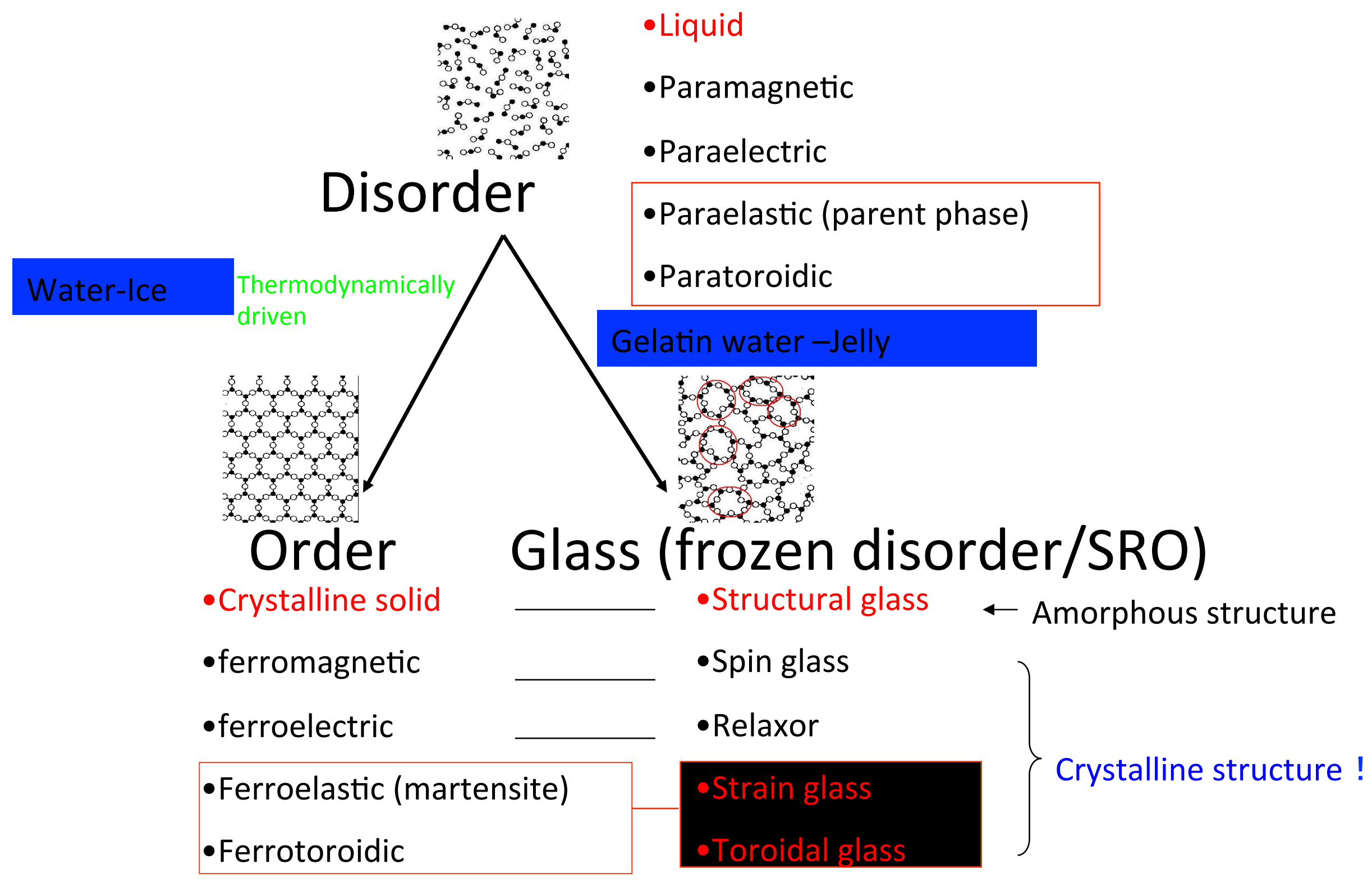




\section{ZFC/FC of strain glass - striking similarity among the three different kinds of glass}

(b)
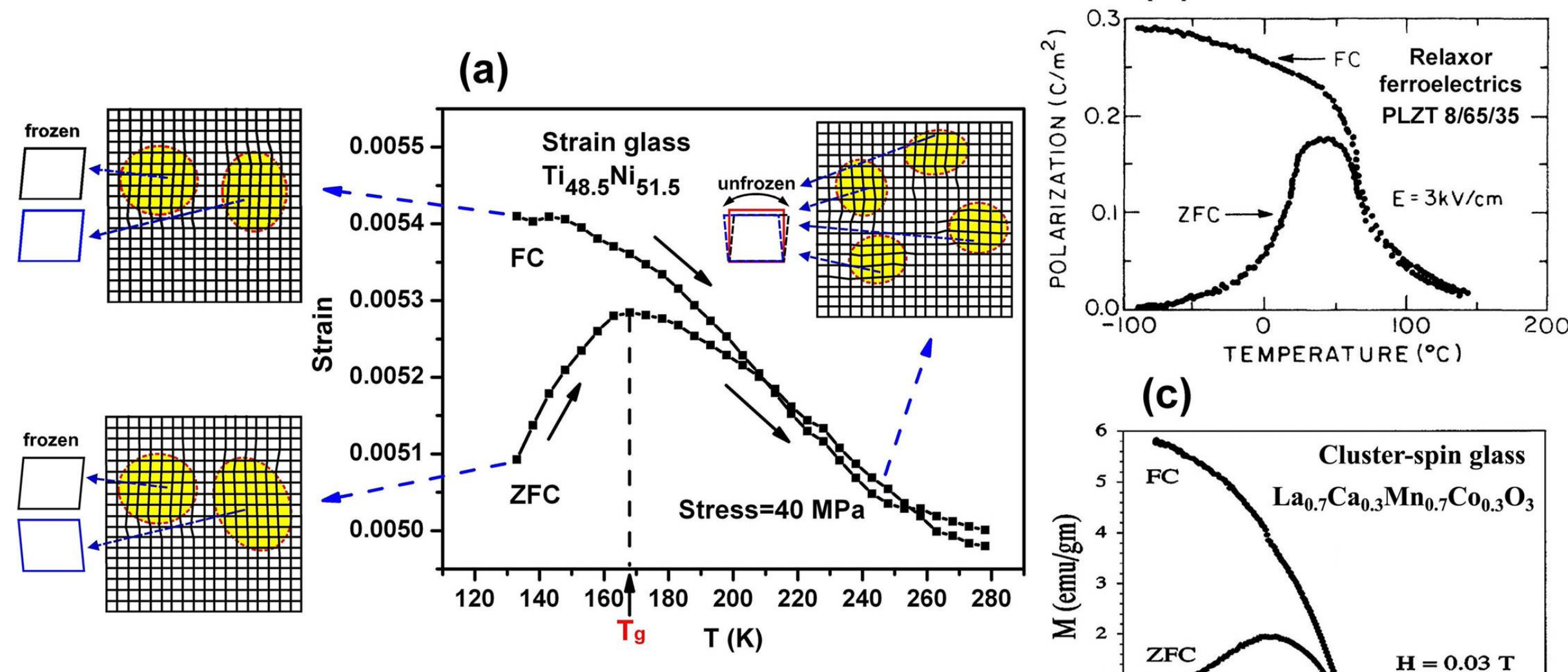

(c)

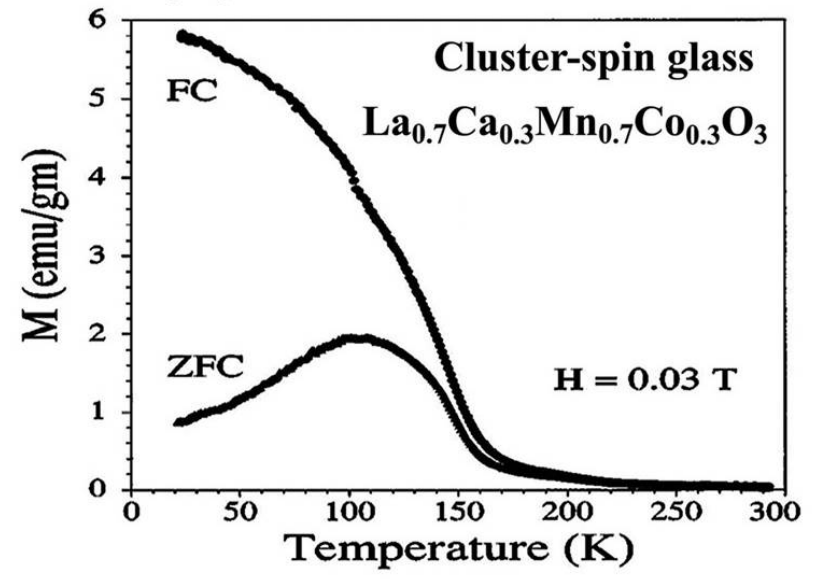

Y. Wang et al., Phys. Rev. B 76, 132201 (2007) 


\section{Local strain ordering in strain glass}

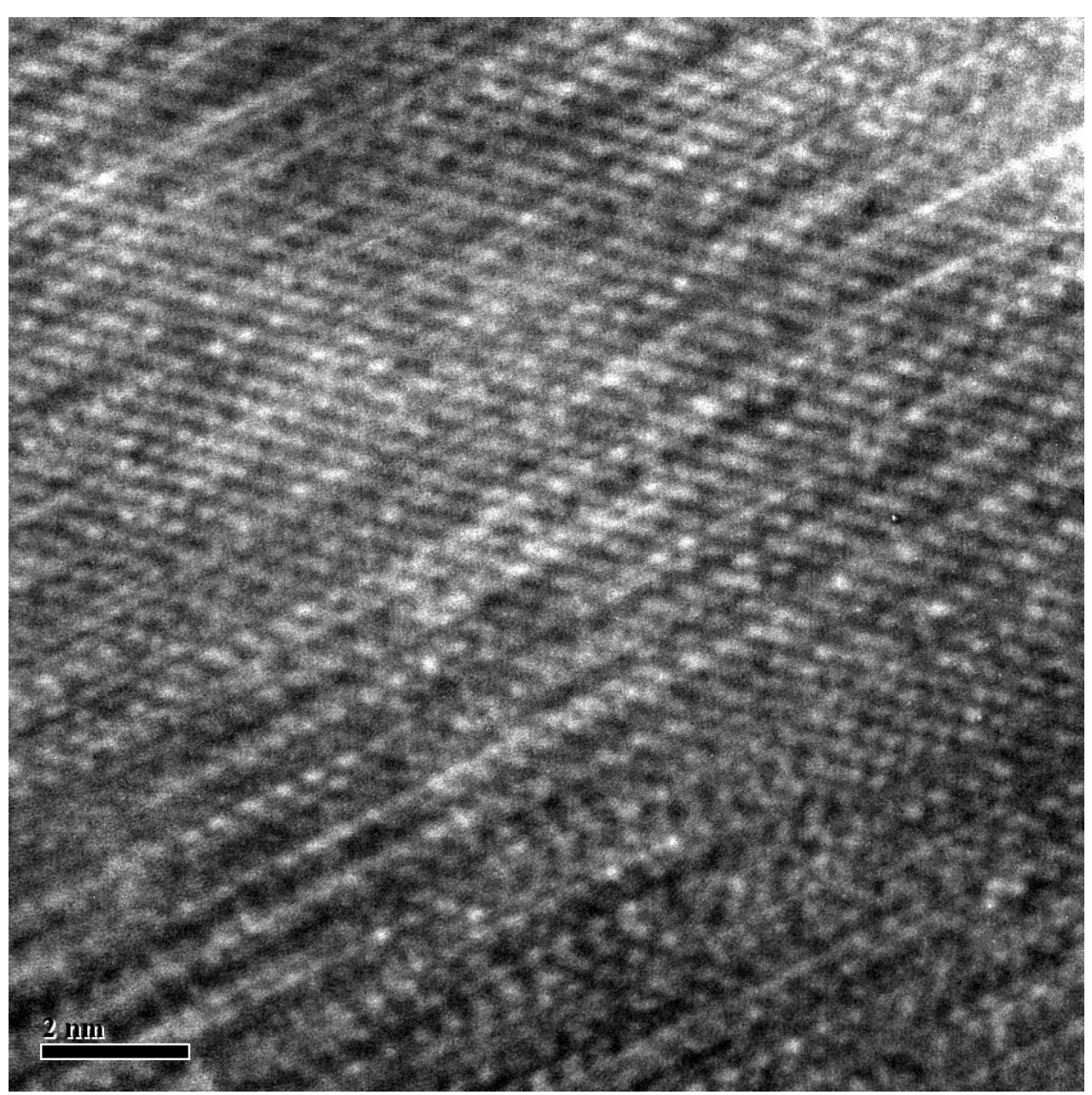

X. Ren et al. (2008): $\mathrm{Ni}_{\mathrm{x}} \mathrm{Ti}_{1-\mathrm{x}}$ 


\section{Nanodomains in PLZT \& PMN relaxors}

Appl. Phys. Lett. 86, 202907 (2005)
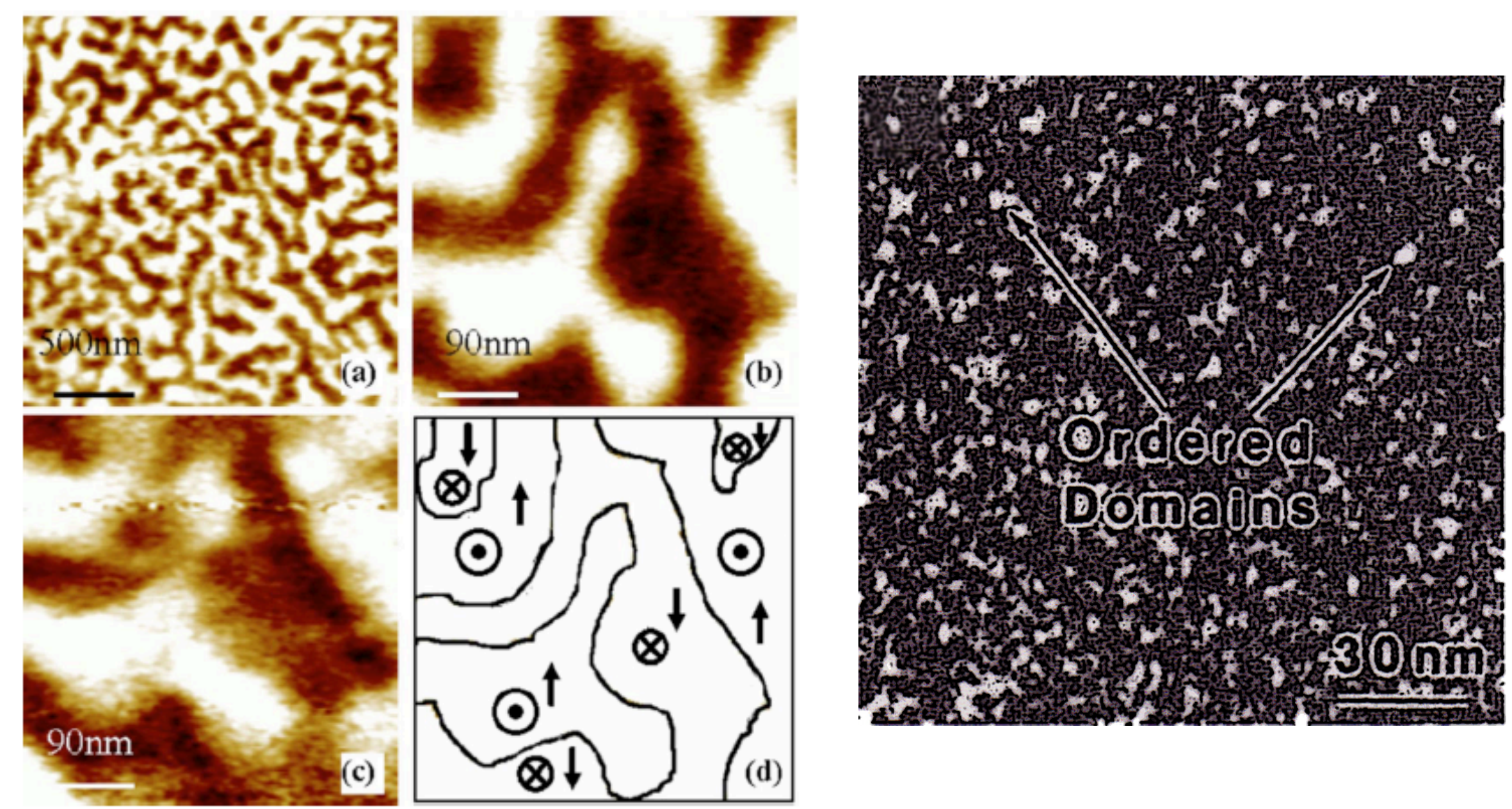

V.V. Shvartsman et al. 


\section{Local strain ordering in strain glass}

PRL 112, 025701 (2014)

PHYSICAL REVIEW LETTERS

week ending 17 JANUARY 2014

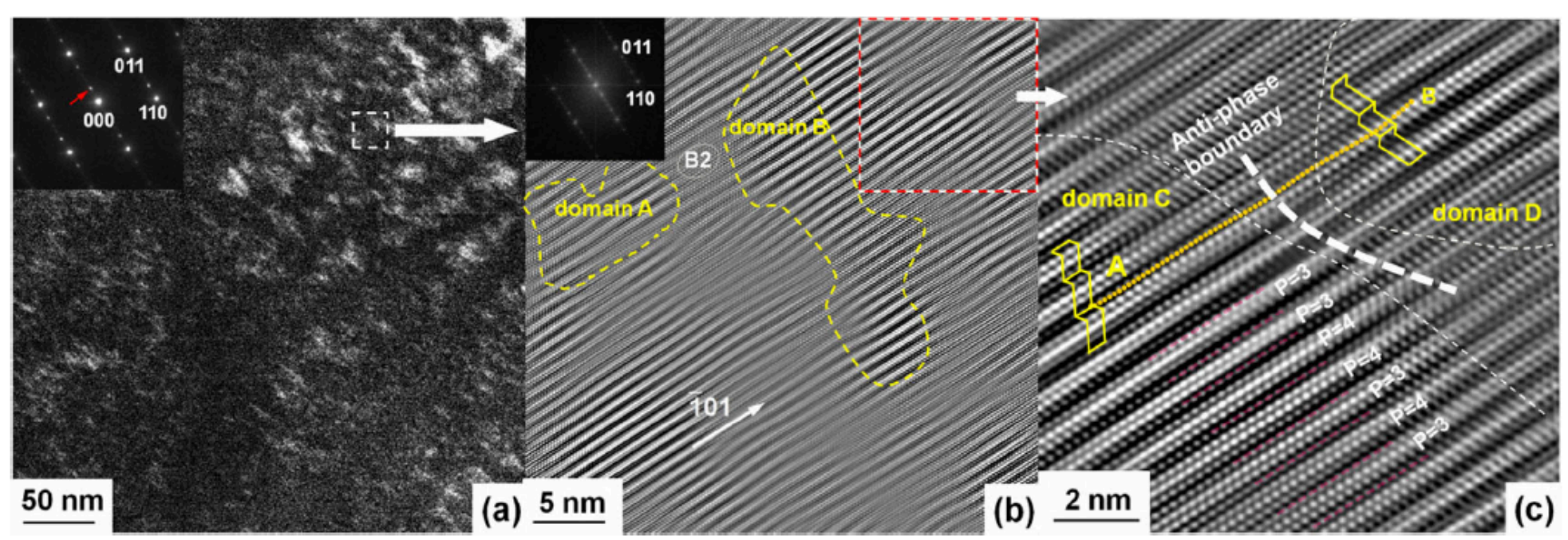

Y. Zhou et al., $\mathrm{Ti}_{50} \mathrm{Pd}_{41} \mathrm{Cr}_{9}$ 


\section{Simulations of strain glass: anisotropy}

(I) $\mathrm{A}_{3}=4.54$

(II) $\mathrm{A}_{3}=0.50$

(III) $\mathrm{A}_{3}=0.05$

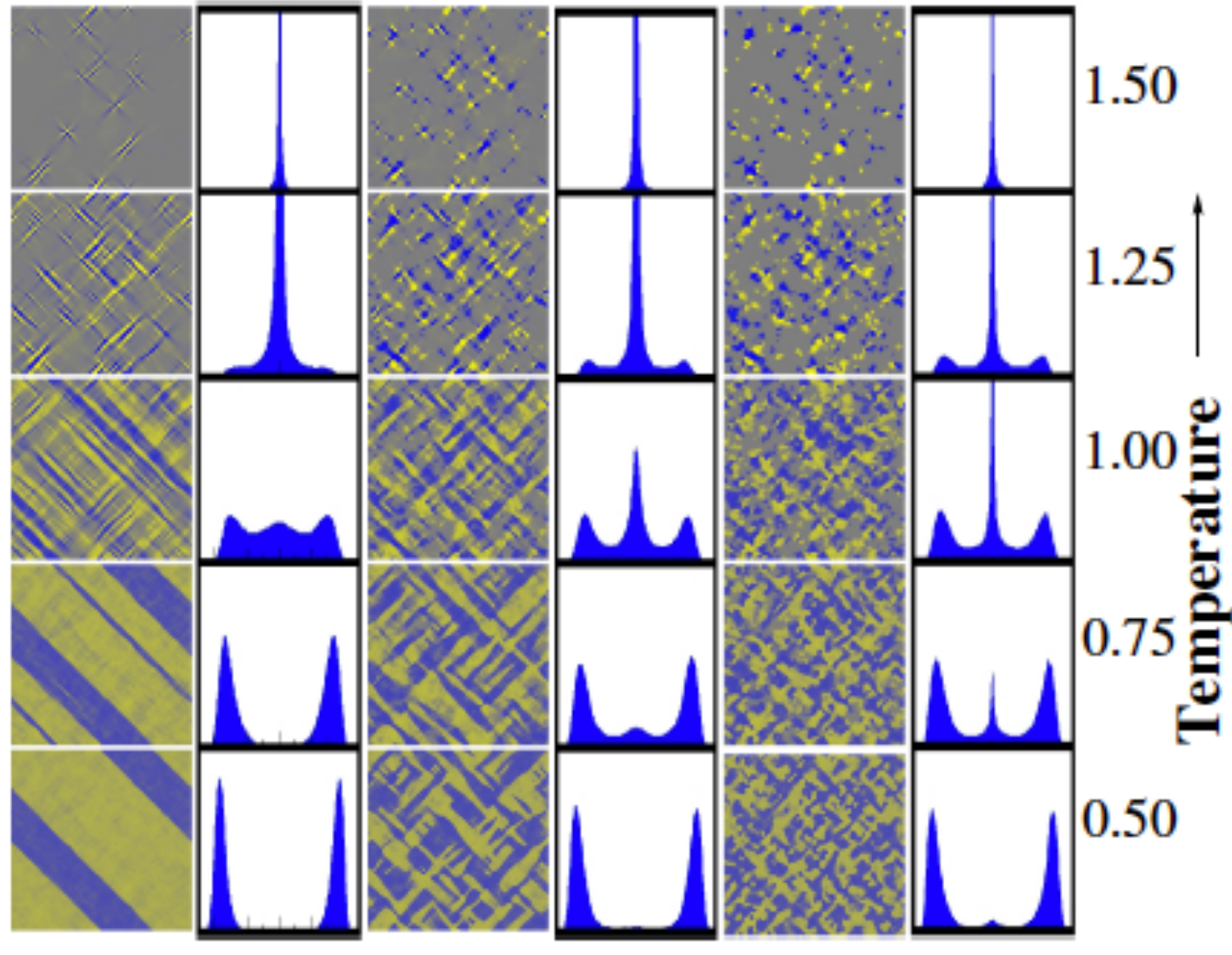

Elastic anisotropy: $\mathrm{A}=2 \mathrm{C}_{44} /\left(\mathrm{C}_{11}-\mathrm{C}_{22}\right)$

P. Lloveras et al., Phys. Rev. Lett. 100, 165707 (2008)
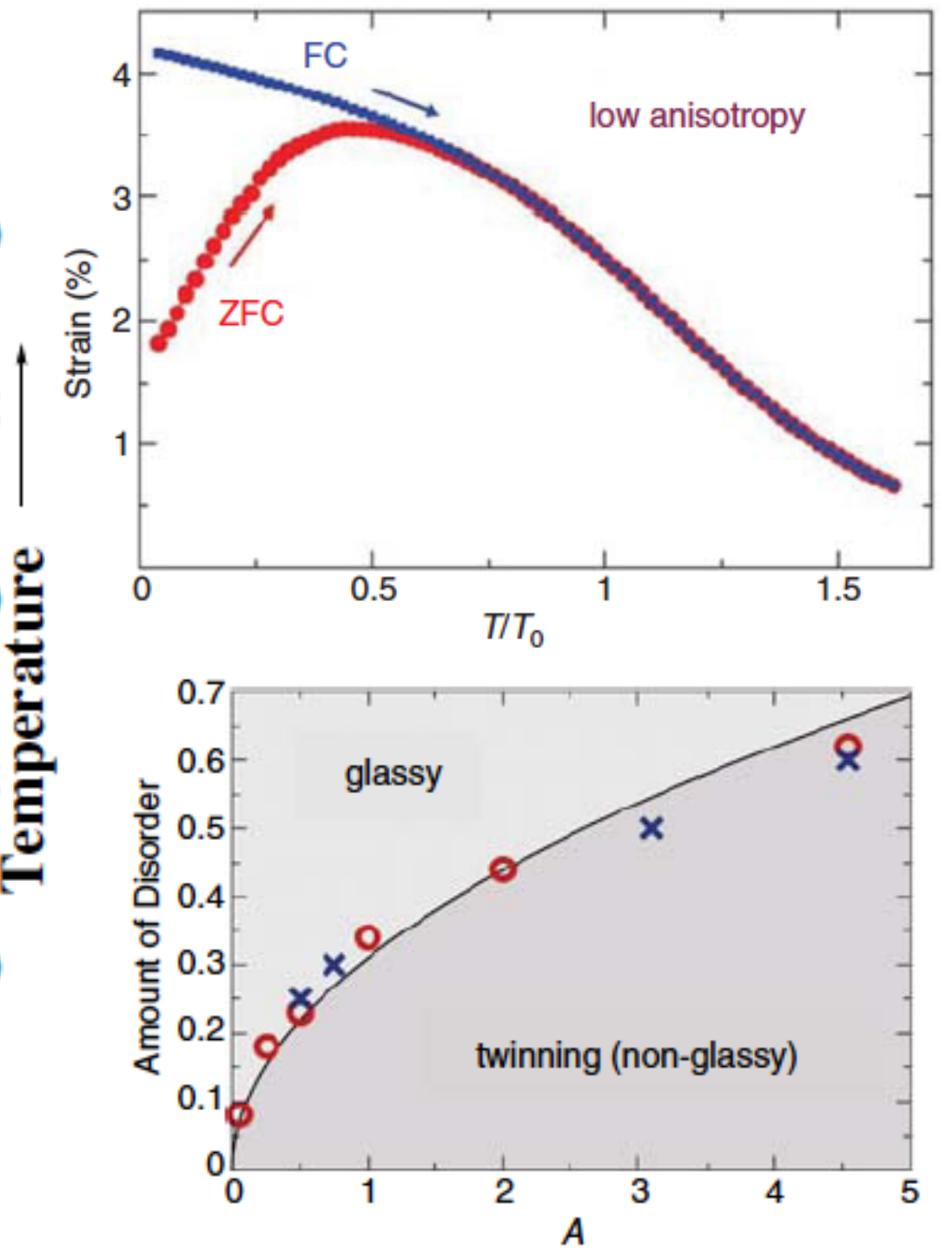

MRS Bulletin p.838 (Nov. 2009) 


\section{Criticality and the piezoelectric response of ferroelectrics}

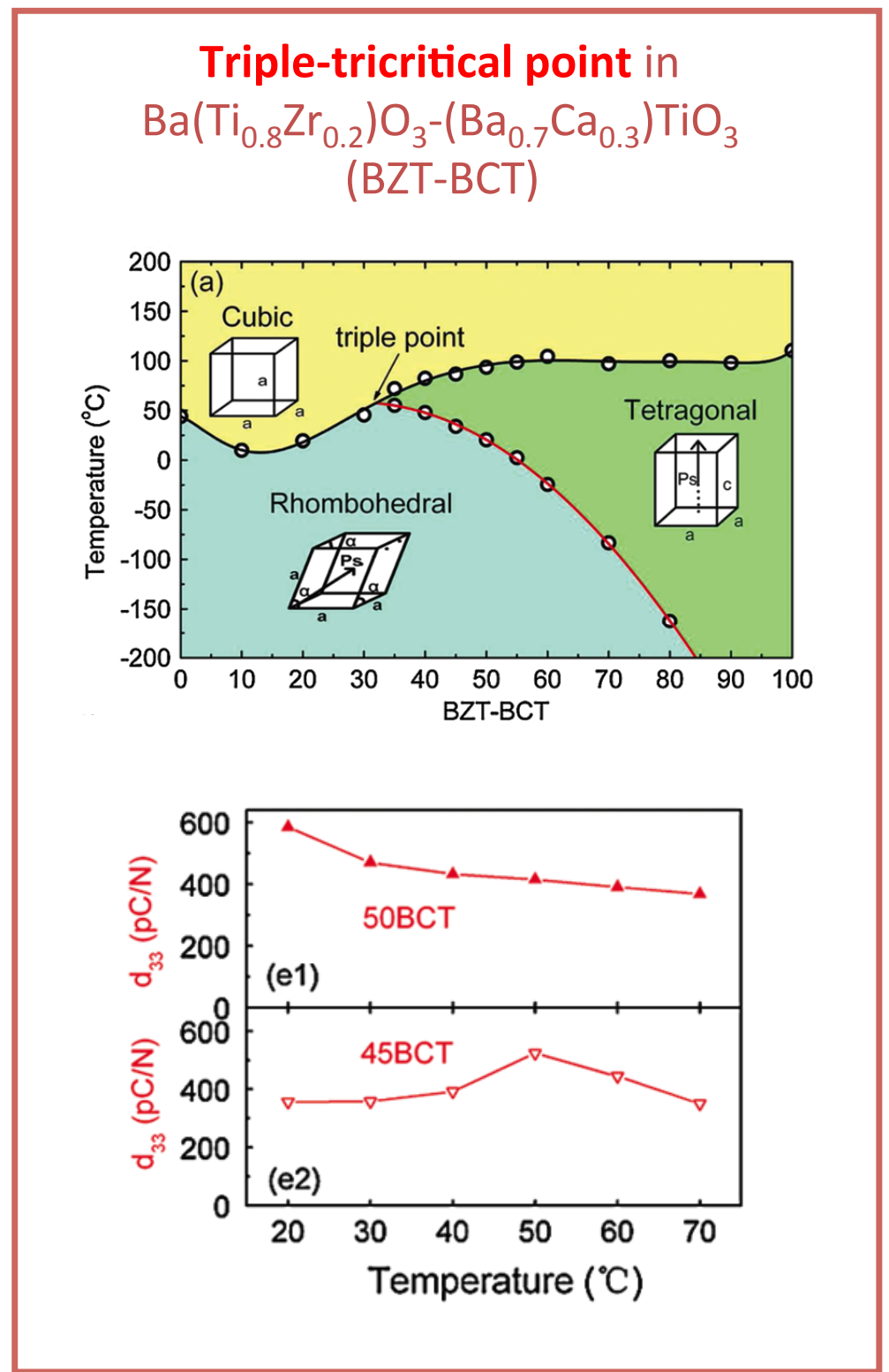

W. Liu and X. Ren, Phys. Rev. Lett. 103, 257602 (2009) 


\section{Extended 2D Ginzburg-Landau model for $\mathrm{BaTiO}_{3}$}

$$
G=G_{G L}+G_{e m}+G_{e s}
$$

Ginzburg-Landau free energy (Li et al. J. Appl. Phys. 98, 064101 (2005))

$$
G_{G L}=\frac{1}{2} a_{2}\left(T-T_{C}\right) P^{2}+\frac{1}{4} a_{4}(\vec{n}) P^{4}+\frac{1}{6} a_{6}(\vec{n}) P^{6}+\frac{1}{8} a_{8}(\vec{n}) P^{8}+G_{G}(\nabla \vec{P})-\vec{P} \cdot \vec{E}_{e x t}
$$

- Electromechanical energy (Elastic energy + Polarization-Strain coupling)

$$
G_{e m}=\frac{1}{2} A_{1} e_{1}^{2}+\frac{1}{2} A_{2} e_{2}^{2}+\frac{1}{2} A_{3} e_{3}^{2}+\alpha e_{1}\left(P_{x}^{2}+P_{y}^{2}\right)+\beta e_{2}\left(P_{x}^{2}-P_{y}^{2}\right)+\gamma e_{3} P_{x} P_{y}-e_{0} \sigma_{1}
$$

- Electrostatic energy

$$
G_{e s}=-\frac{1}{2} \vec{P} \cdot \vec{E}=\frac{\varepsilon_{0}}{2} E^{2} \longrightarrow \int G_{e s} d \vec{r}=\frac{1}{2 \varepsilon_{0}} \int \frac{d \vec{k}}{(2 \pi)^{2}} \frac{|\vec{k} \cdot \vec{P}|^{2}}{k^{2}}
$$




\section{Piezoelectric response near the $\mathrm{C}-\mathrm{T}$ transition of [001] poled $\mathrm{BaTiO}_{3}$}

\section{Clean limit: No disorder}
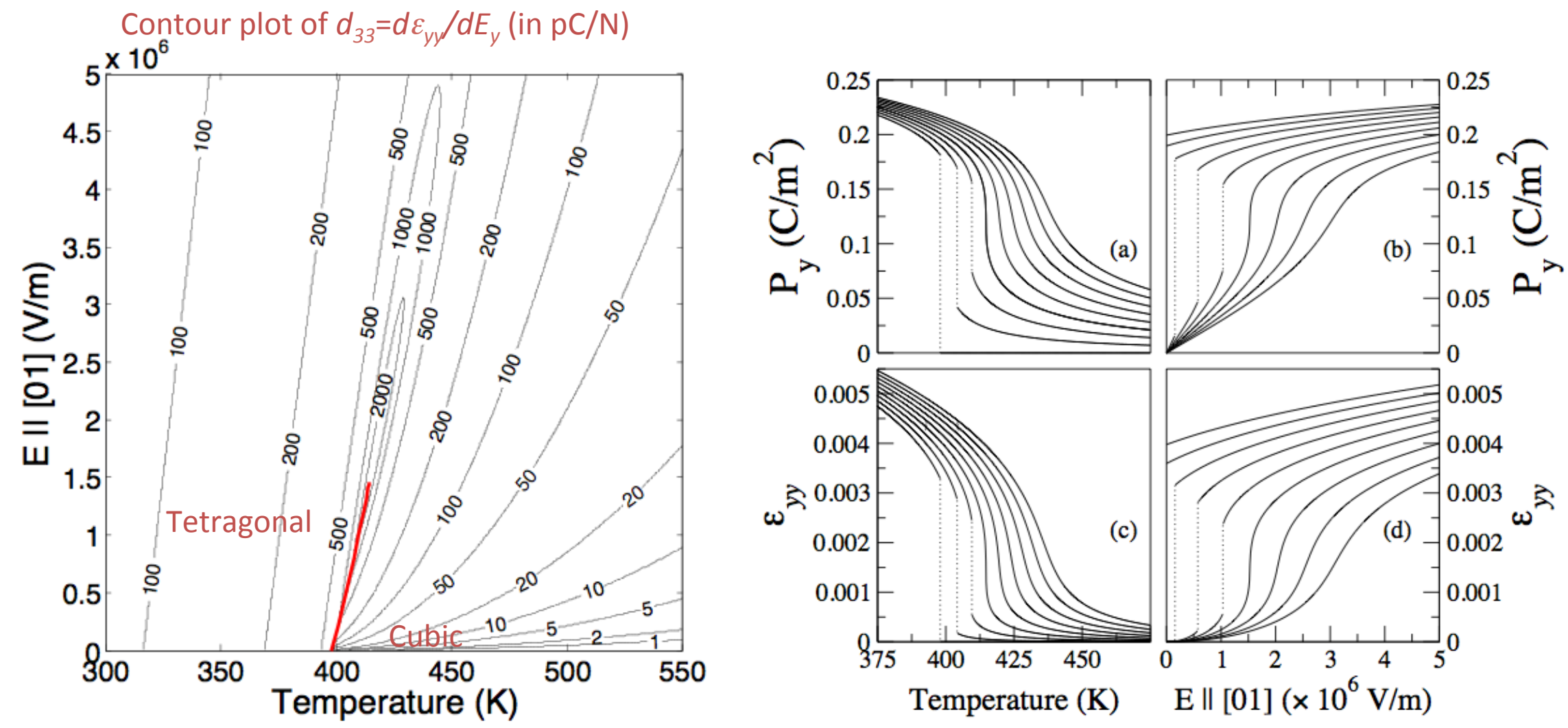

- Divergence of $d_{33}$ at the critical point

- $\vec{P} \| \vec{E}$

- Soft potential with respect to the modulus of $\vec{P}$ 
Piezoelectric response near the C-T transition of [001] poled $\mathrm{BaTiO}_{3}$

Small disorder: $\sqrt{\left\langle\sigma_{1}^{2}\right\rangle}=2.0 \times 10^{9} \mathrm{~N} / \mathrm{m}^{2}$

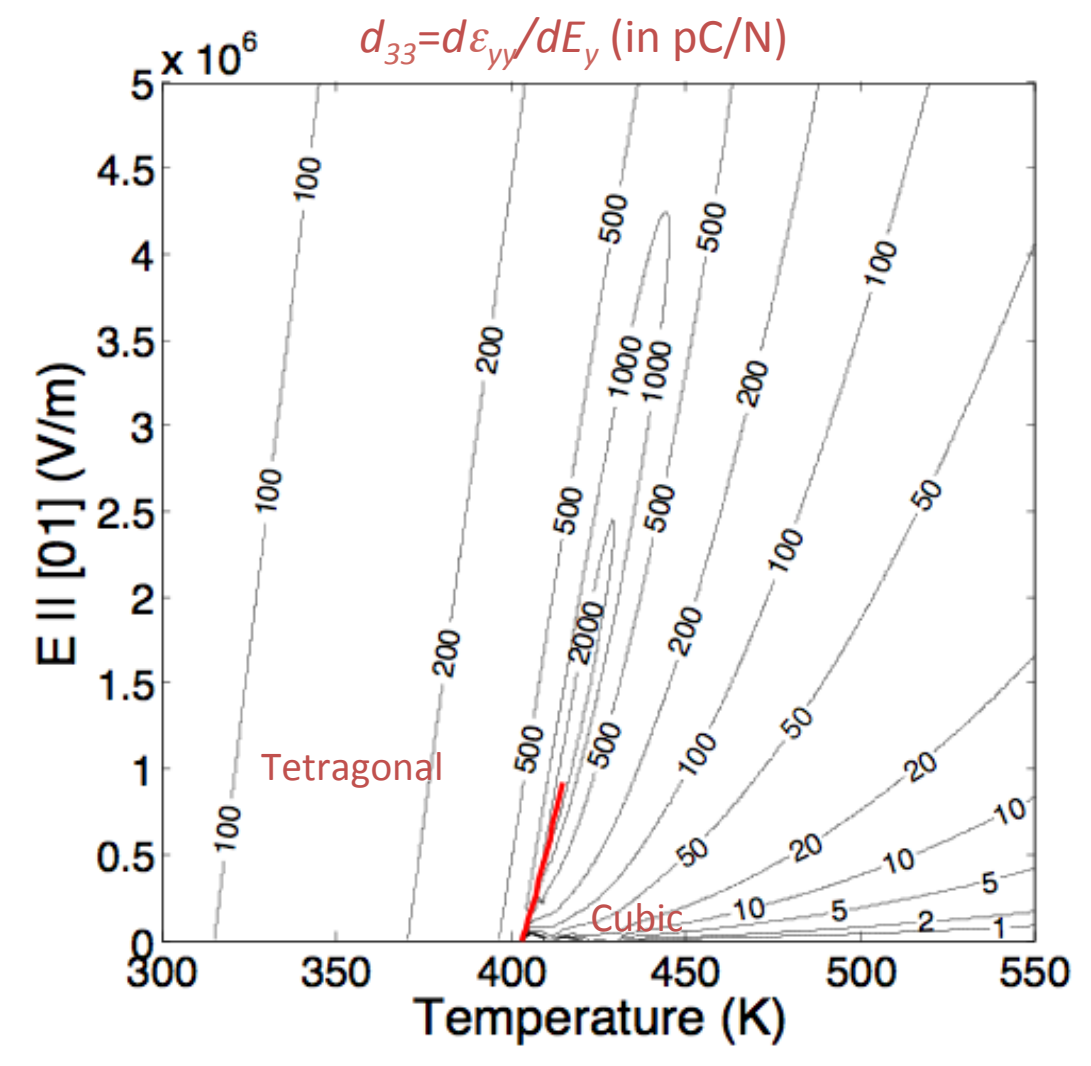

- Smaller critical electric field (with respect to the clean limit)

- Divergence of $d_{33}$ at the critical point

- No broadening of the region where $d_{33}$ is large 
Piezoelectric response near the $\mathrm{C}-\mathrm{T}$ transition of [001] poled $\mathrm{BaTiO}_{3}$

Large disorder: $\sqrt{\left\langle\sigma_{1}^{2}\right\rangle}=4.5 \times 10^{9} \mathrm{~N} / \mathrm{m}^{2}$
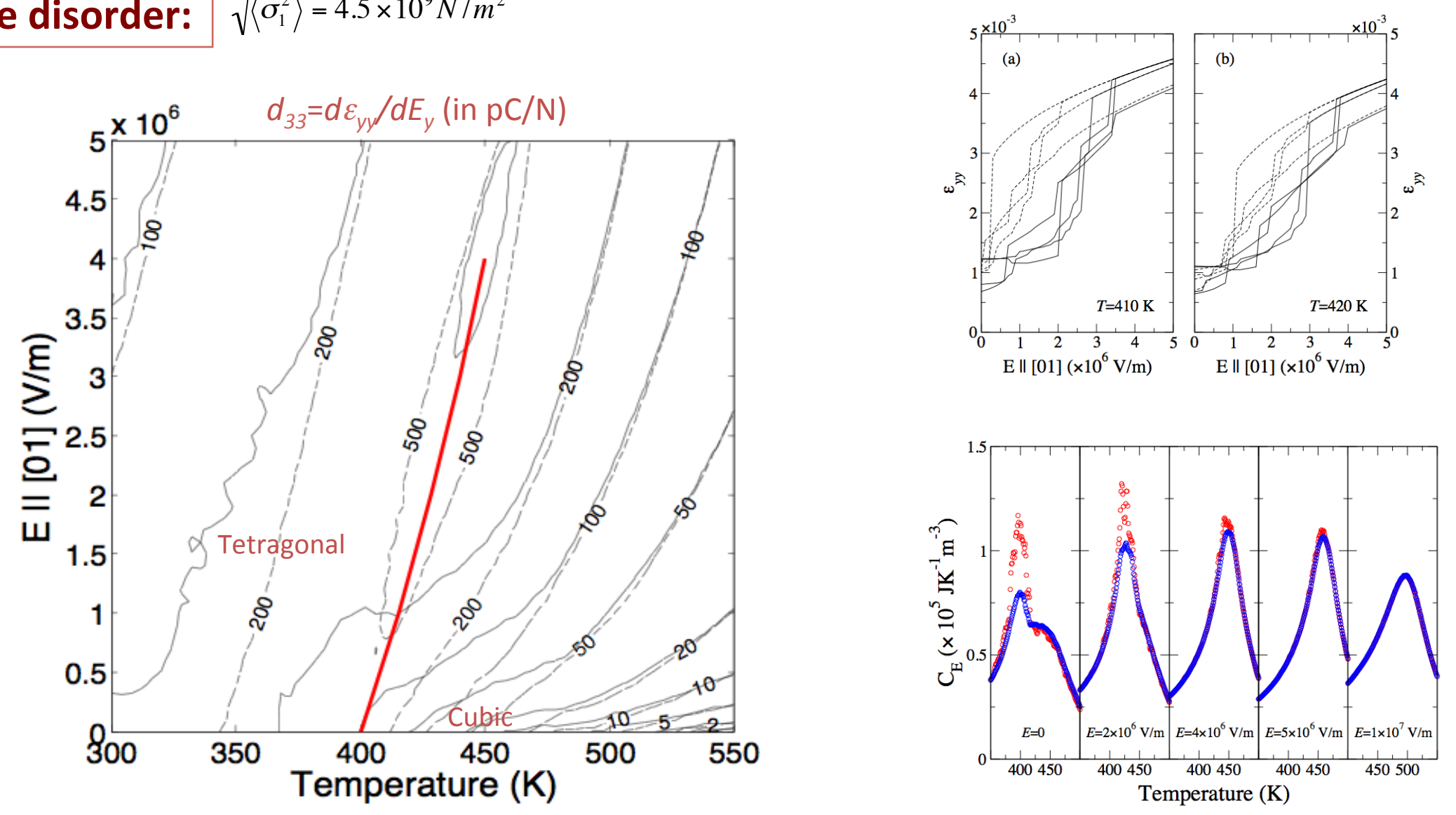

- Existence of a critical point

- $d_{33}$ is maximum near the critical point

- $d_{33}^{33}$ does not diverge at the critical point

\section{RELAXOR!}




\section{MULTIFERROIC TRANSITIONS}


Hexagonal $\mathrm{RMnO}_{3}: \mathrm{R}=\mathrm{Ho}, \mathrm{Er}, \mathrm{Tm}, \mathrm{Yb}, \mathrm{Lu}, \mathrm{Y}, \mathrm{Sc}$

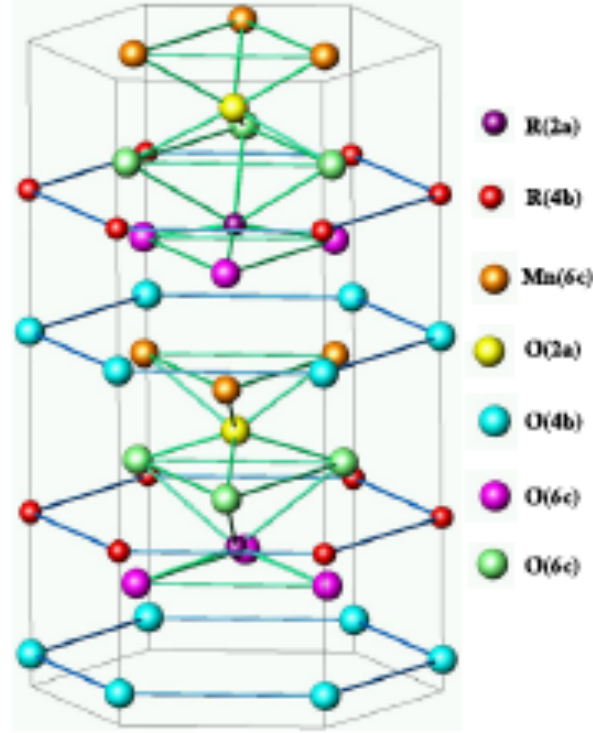

(a)
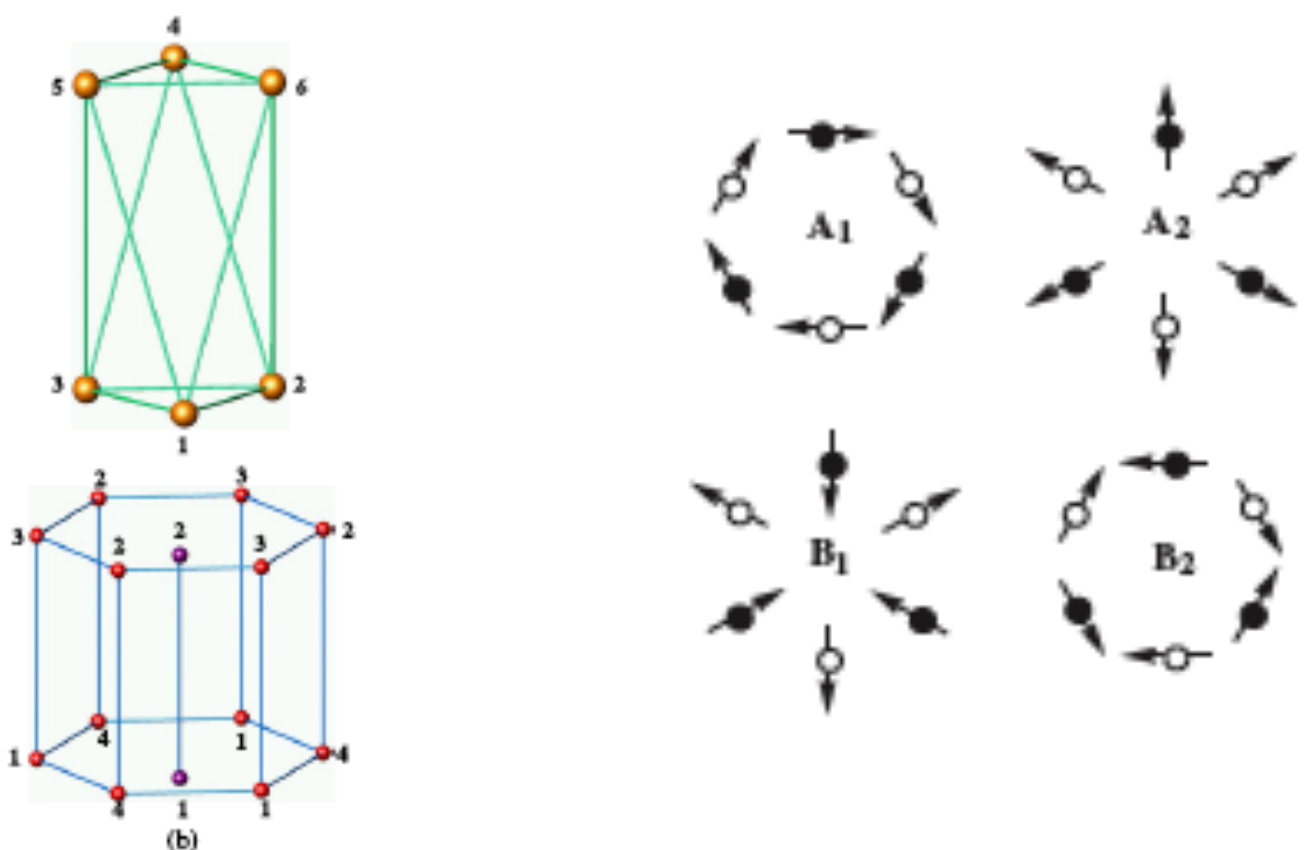

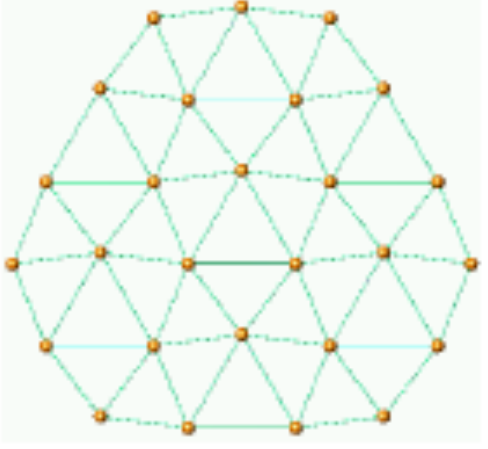

(a)

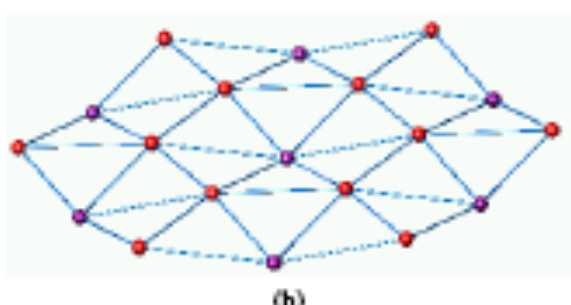

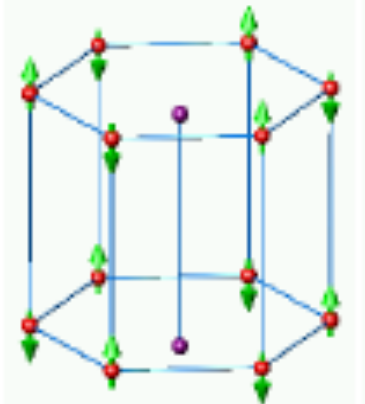

(a)

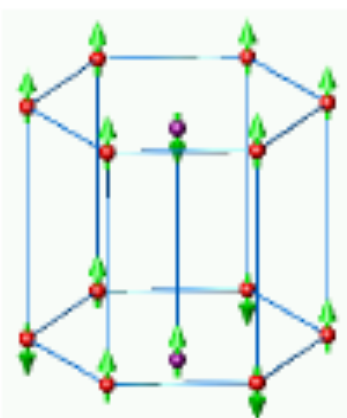

(b)

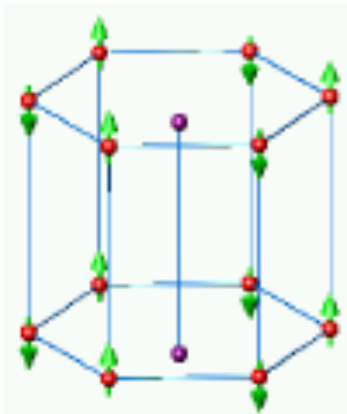

(c)

Th. Lonkai et al., JAP 93, 8191 (2003): Weak interlayer coupling

I. Munawar, S.S. Curnoe, J. Phys. Cond. Matt. 18, 9575 (2006) 


\section{Magnetic Structure and SHG Selection Rules}

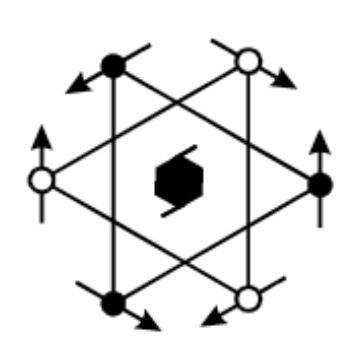

$\mathrm{P} \underline{6}_{3} \underline{\underline{c m}}\left(\mathrm{~B}_{2}\right)$

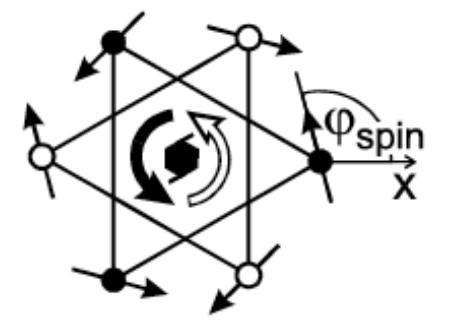

$\mathrm{P} \underline{6}_{3}$ (B)

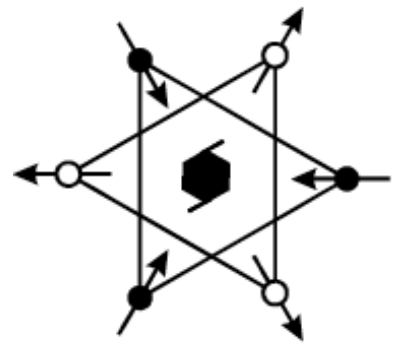

$\mathrm{P} \underline{6}_{3} \mathrm{Cm}\left(\mathrm{B}_{1}\right)$

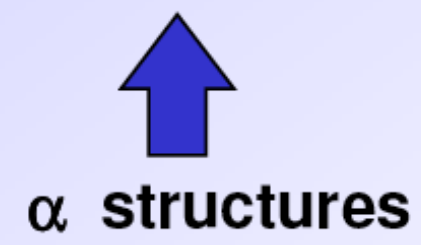

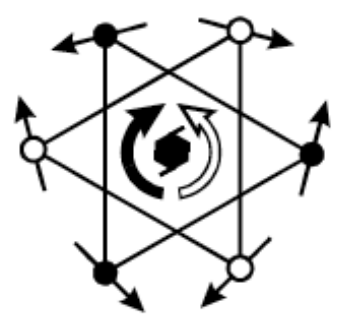

$\mathrm{P} 3 \underline{\mathrm{c}}\left(\mathrm{A}_{2}{ }^{\prime}\right)$
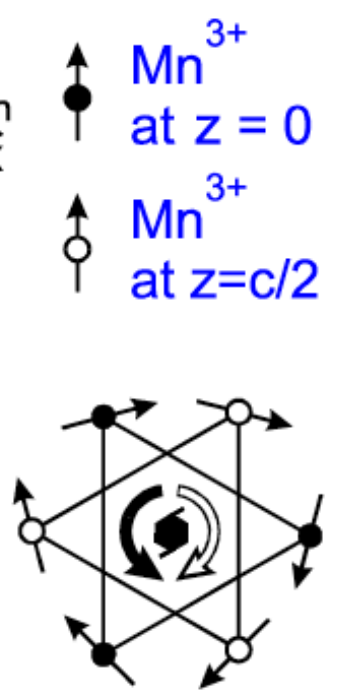

P3c $\left(A_{1}{ }^{\prime}\right)$

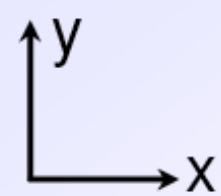

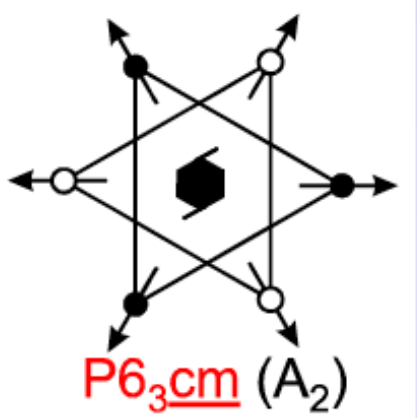

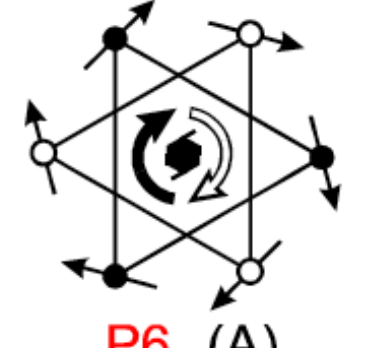

$\mathrm{P}_{3}(\mathrm{~A})$

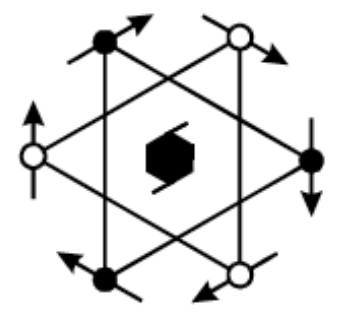

$\mathrm{P}_{3} \mathrm{~cm}\left(\mathrm{~A}_{1}\right)$

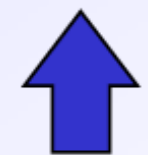

$\beta$ structures
At least 8 different triangular inplane spin structures with different magnetic symmetries and different selection rules for SHG

$\alpha$ structures: SHG for $\mathbf{k} \| \mathbf{z}$ allowed

$\alpha_{\mathrm{x}}\left(\varphi=0^{\circ}\right): \quad \chi_{\mathrm{xxx}}=0, \quad \chi_{\mathrm{yyy}} \neq 0$

$\alpha_{y}\left(\varphi=90^{\circ}\right): \quad \chi_{\mathrm{xxx}} \neq 0, \quad \chi_{\mathrm{yyy}}=0$

$\alpha_{\rho}\left(\varphi=0-90^{\circ}\right): \chi_{x x x} \propto \sin \varphi, \chi_{\text {yyy }} \propto \cos \varphi$

B structures: SHG for $\mathbf{k} \| \mathbf{z}$ not allowed

$\beta_{\mathrm{x}}, \beta_{\mathrm{y}}, \beta_{\rho}: \quad \chi_{\mathrm{xxx}}=0, \quad \chi_{\mathrm{yyy}}=0$

Determine $\beta$ structure from $\alpha-\beta$ transition

$\alpha_{\mathrm{x}} \rightarrow \beta_{\mathrm{y}}: \quad \chi_{\mathrm{xxx}}=0, \quad \chi_{\mathrm{yyy}} \propto \cos \varphi$

$\alpha_{y} \rightarrow \beta_{x}: \quad \chi_{x x x} \propto \sin \varphi, \chi_{y y y}=0$

Contrary to diffraction techniques:

$\alpha$ and $\beta$ models clearly distinguishable!

T. Lottermoser and M. Fiebig (2004) 


\section{SH spectrum and Magnetic Symmetry}

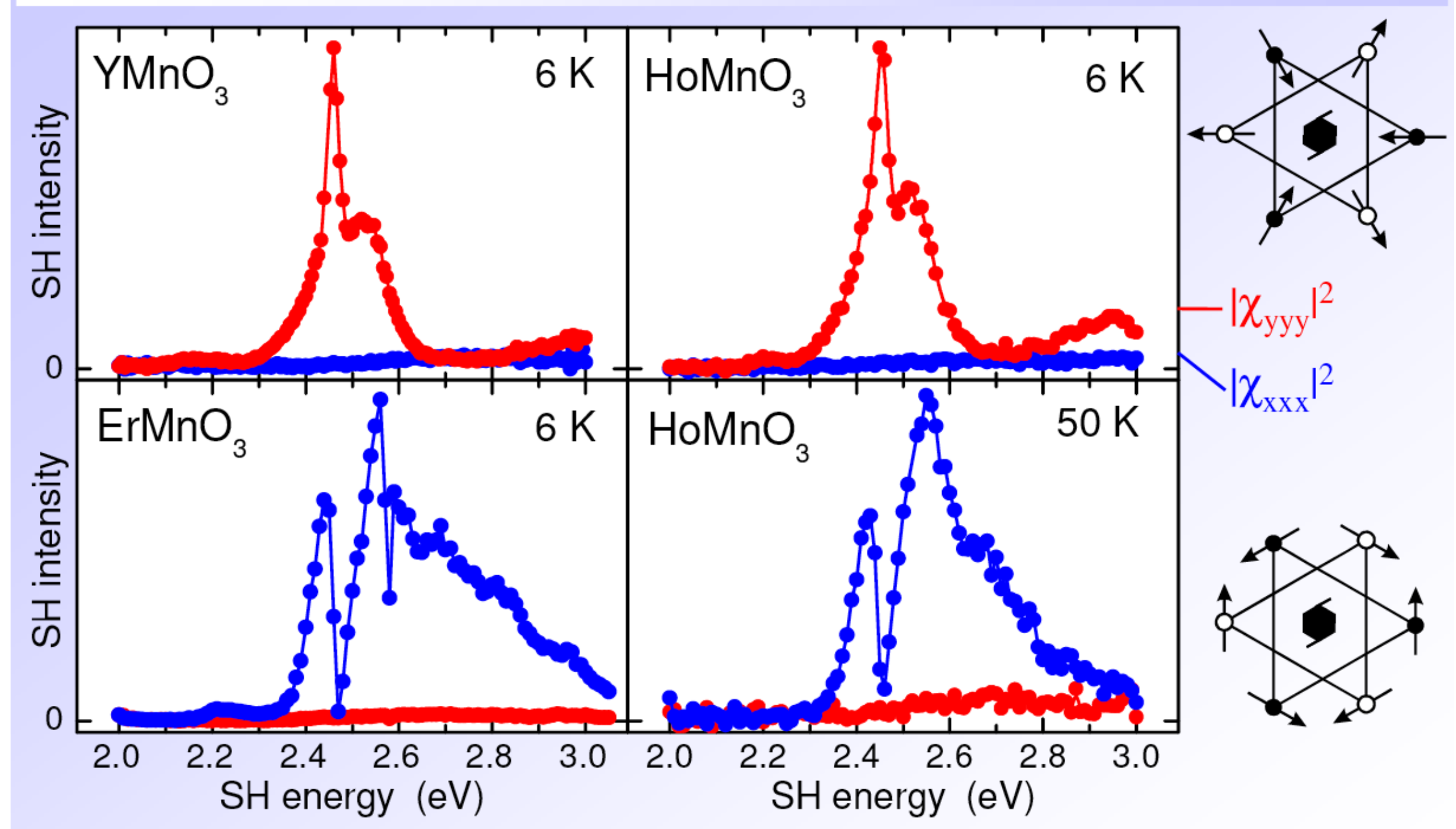

The magnetic symmetry, not the $\mathrm{R}$ ion, determines the $\mathrm{SH}$ spectrum of $\mathrm{RMnO}_{3}$ 


\section{Free Energy for a 2D Multiferroic Transition}

$$
\begin{gathered}
F\left(M, P_{,}, e\right)= \\
a_{1}\left(M_{1}{ }^{2}+M_{2}{ }^{2}\right)+b_{1}\left(M_{1}{ }^{2}+M_{2}{ }^{2}\right)^{2}+c_{1}\left(M_{1}{ }^{4}+M_{2}{ }^{4}\right) \\
+a_{2}\left(P_{1}{ }^{2}+P_{2}{ }^{2}\right)+b_{2}\left(P_{1}{ }^{2}+P_{2}{ }^{2}\right)^{2}+c_{2}\left(P_{1}{ }^{4}+P_{2}{ }^{4}\right) \\
+a_{3}\left(e_{2}{ }^{2}+e_{3}{ }^{2}\right)+b_{3}\left(e_{2}{ }^{3}-3 e_{2} e_{3}{ }^{2}\right)+c_{3}\left(e_{2}{ }^{2}+e_{3}{ }^{2}\right)^{2} \\
+A\left[e_{2}\left(P_{1}{ }^{2}-P_{2}{ }^{2}\right)+e_{3} P_{1} P_{2}\right] \\
+B\left[e_{2}\left(M_{1}{ }^{2}-M_{2}{ }^{2}\right)+e_{3} M_{1} M_{2}\right] \\
+C\left[\left(P_{1}{ }^{2}+P_{2}{ }^{2}\right)\left(M_{1}{ }^{2}+M_{2}{ }^{2}\right)\right] \\
+ \text { gradient terms }
\end{gathered}
$$




\section{Gradient couplings: domains \& vortices}

$$
\begin{aligned}
& F_{G}=M^{2}(\nabla \cdot P)+M \cdot[(M \cdot \nabla) P] \\
& +P \cdot[M(\nabla \cdot M)+P \cdot[M \times(\nabla \times M)] \\
& +\varepsilon(\nabla \cdot M)^{2}+\varepsilon(\nabla \times M)^{2} \\
& +\varepsilon(\nabla \cdot P)+P \cdot(\nabla \varepsilon)+\varepsilon(\nabla \times P)^{2}
\end{aligned}
$$

Microstructure simulations underway 


\section{DISLOCATIONS IN MAGNETIC CRYSTALS}

EDGE: AFM spin ordering

SCREW: spiral spin distribution
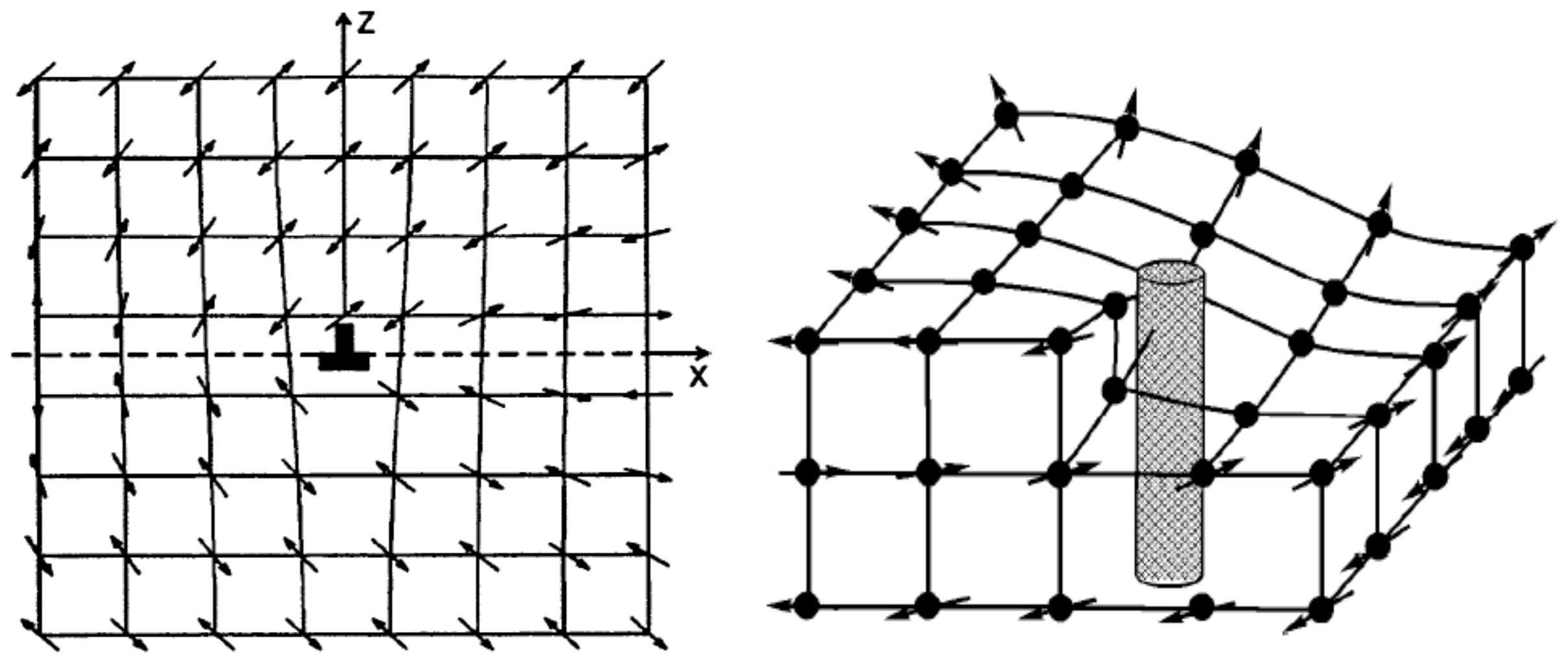


\section{BIOFERROICS}

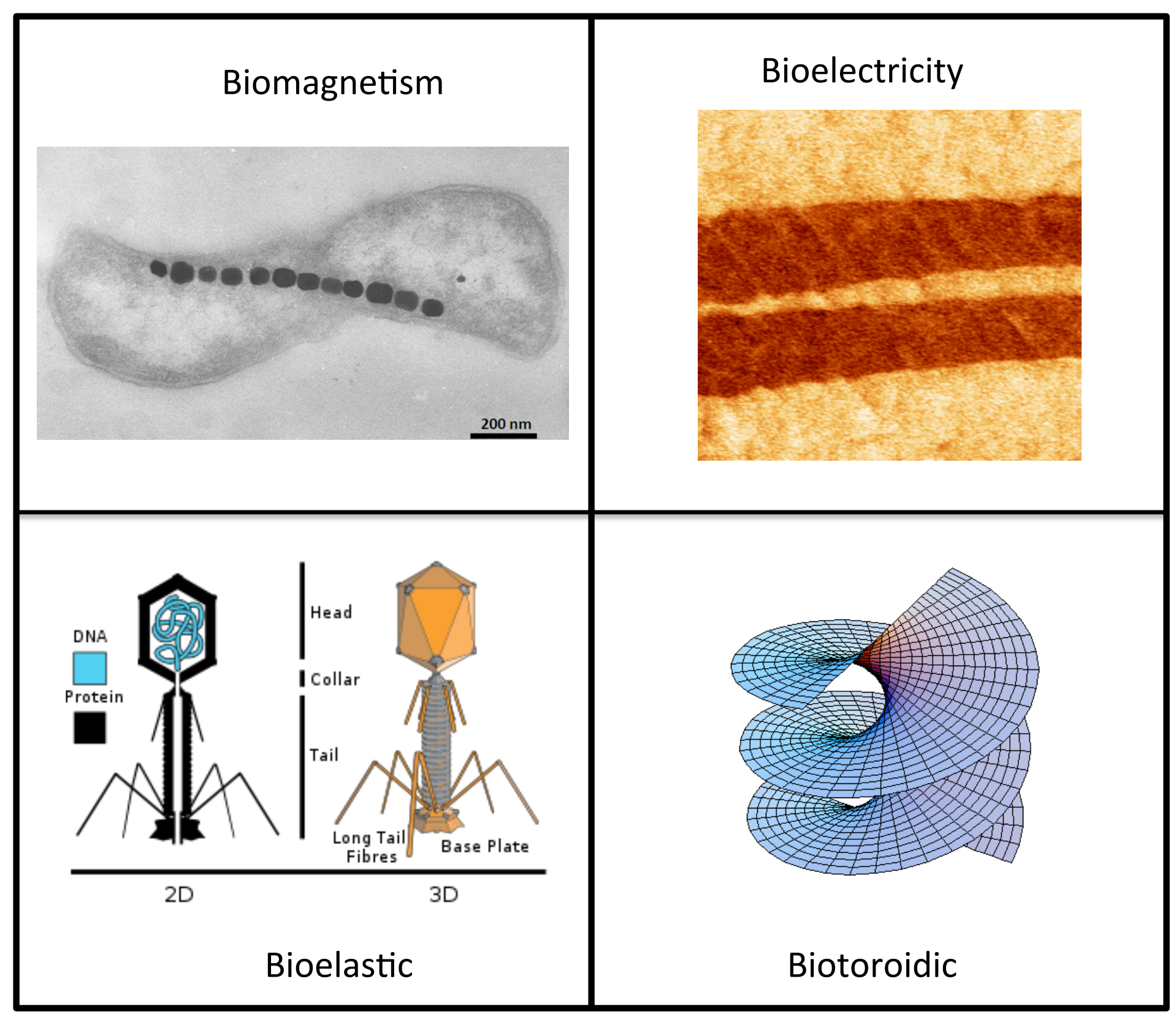




\section{BIOFERROICS}

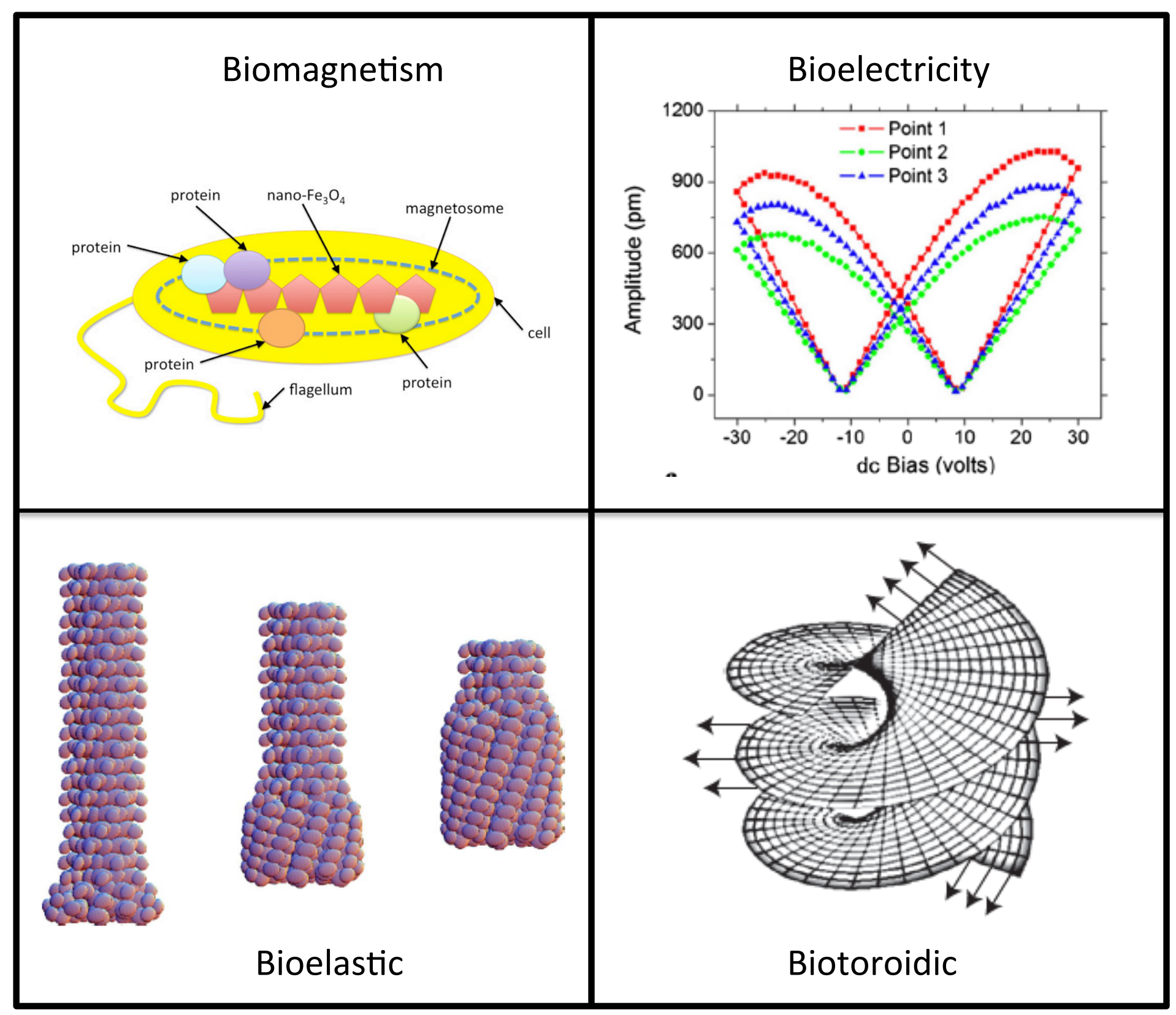




\section{Conclusions}

- Ferroelastics: strain as the order parameter.

- Microstructure: elastic compatibility.

- Magnetic shape memory: coupling to strain.

- Ferroics: Ferrotoroidicity; fourth primary ferroic.

- Various dipole, and long-range interactions.

- Ubiquity of glassy behavior (disorder): strain glass.

- Ferroic phenomena in biological systems. 



\section{Nanodomains in PMN relaxor}

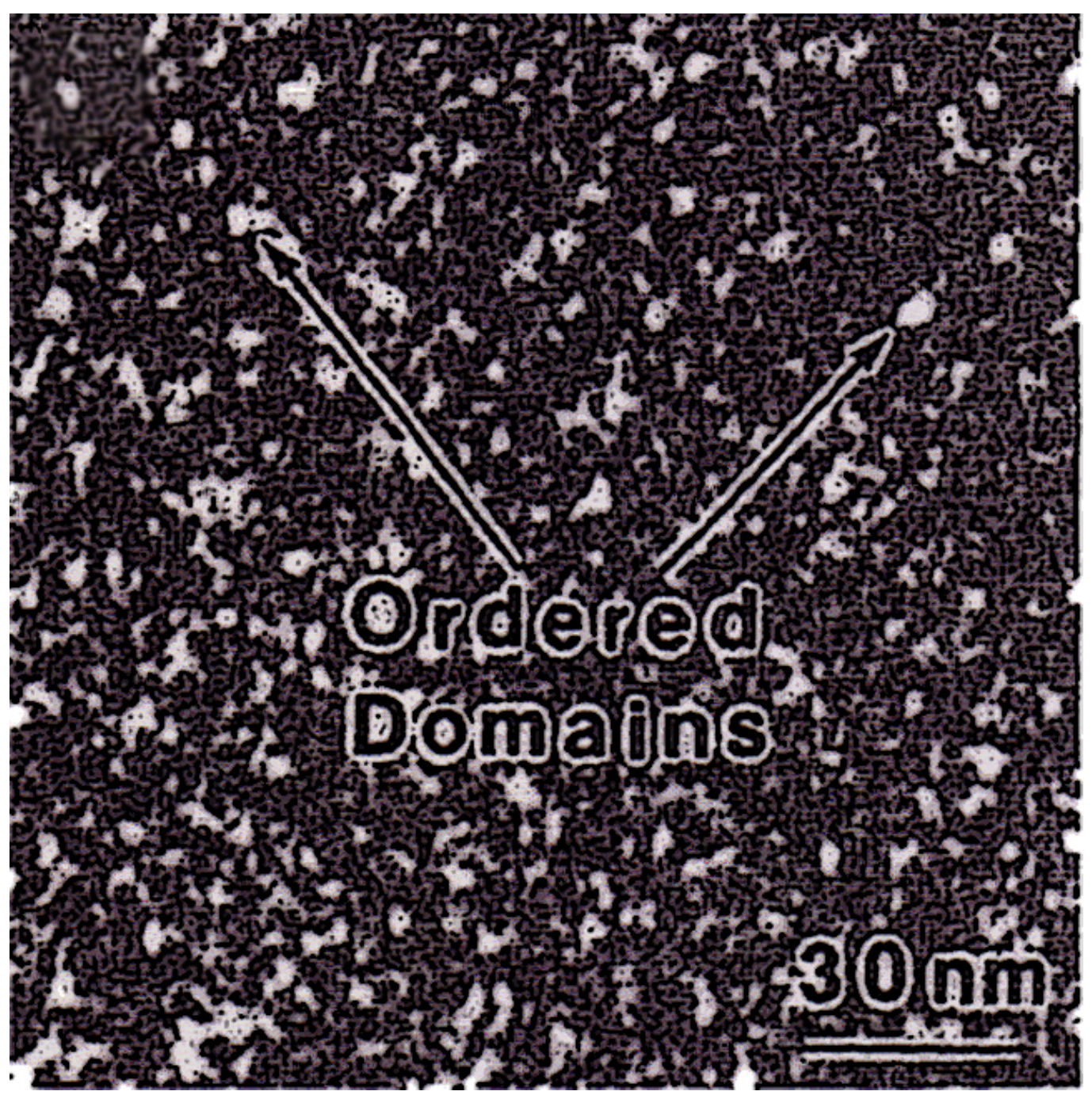




\section{DISPLACIVE MARTENSITIC TRANFORMATIONS}

- First order, solid-solid structural phase transformation.

Ex. Cubic (austenite) to Tetragonal (martensite) transition in FePd alloys.

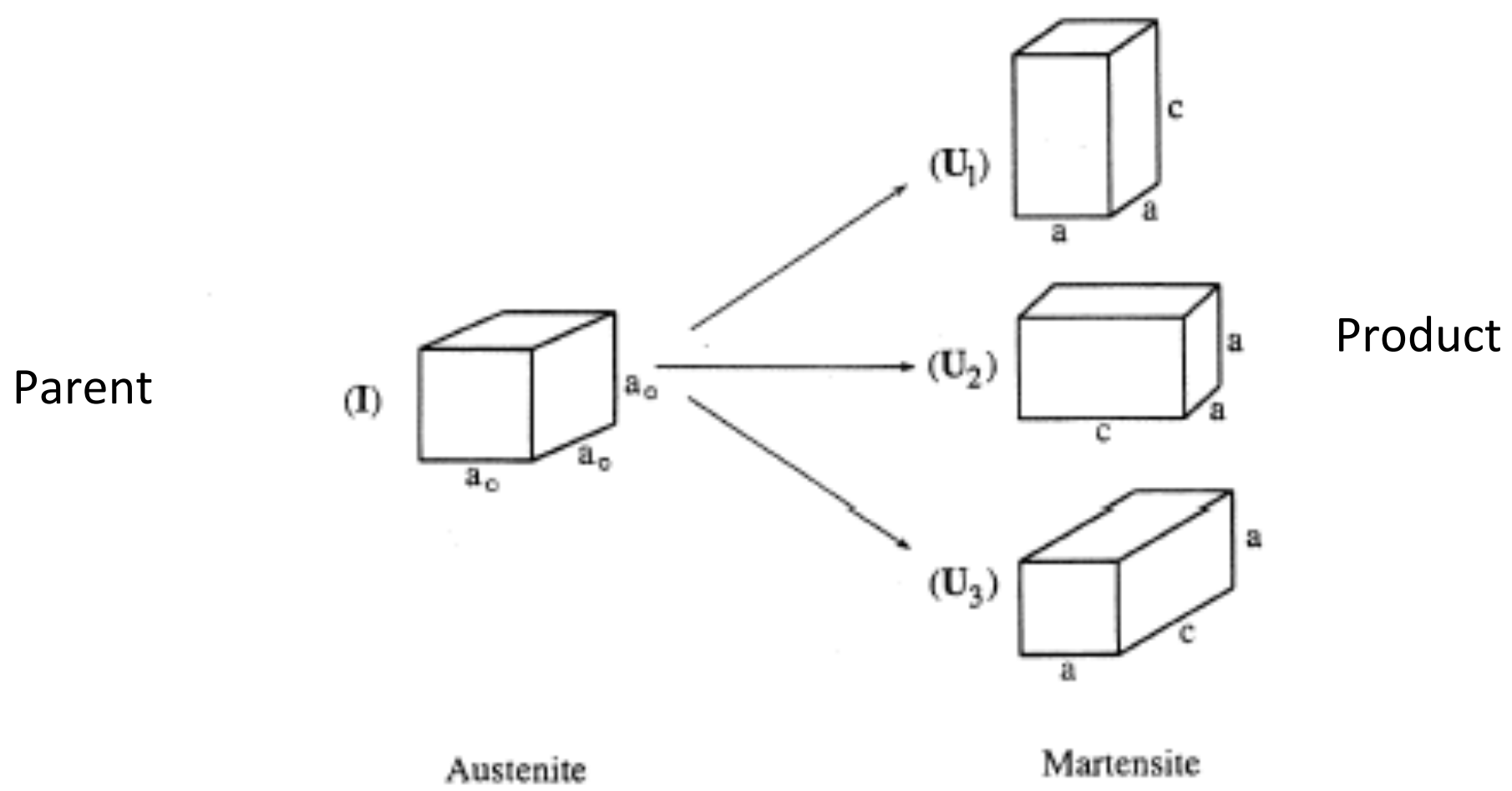

Figure 4.4: The three variants of martensite in a cubic to tetragonal transformation. 


\section{Parameters from experiments}

- Lattice parameters

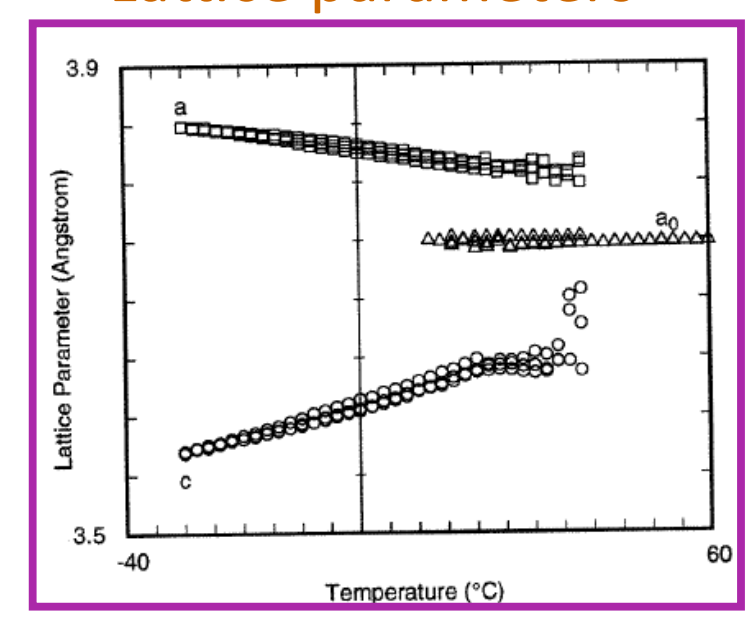

$$
\begin{array}{cc}
\eta_{1}=\frac{a_{t}-a_{o}}{a_{o}}=.0176 & \eta_{3}=\frac{a_{c}-a_{o}}{a_{o}}=-.0335 \\
\varepsilon_{x x}=\varepsilon_{y y} & \varepsilon_{z z}
\end{array}
$$

$$
e_{2}=0 ; e_{3}=\varepsilon_{0}=\frac{2\left(\eta_{1}-\eta_{3}\right)}{\sqrt{6}} ; e_{1}=\frac{2 \eta_{1}+\eta_{3}}{\sqrt{3}}
$$

$$
\frac{A_{B}}{2} e_{1}^{2}+\mathbf{E} e_{1}\left(e_{2}^{2}+e_{3}^{2}\right)=0 \rightarrow e_{1}=-\frac{\mathbf{E}}{A_{B}}\left(e_{2}^{2}+e_{3}^{2}\right)
$$

- Elastic constant

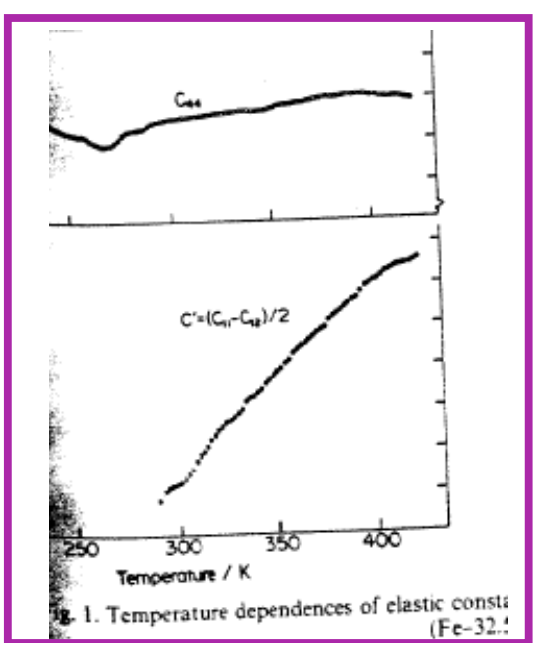

$$
\begin{gathered}
\mathbf{A}_{\mathbf{0}}=\frac{\mathbf{B}^{2}}{\mathbf{U}^{\prime}} \quad \varepsilon_{0}=\frac{-\mathbf{B}}{2 \mathbf{C}^{\mathbf{}}} \\
\because=\mathbf{C}-\frac{\mathbf{E}^{2}}{A_{B}}
\end{gathered}
$$


- Phonon dispersion

$$
\left[\frac{\omega}{\sin (\pi k / 2)}\right]^{2}=\mathbf{A}_{\mathbf{o}}\left(T-\mathbf{T}_{\mathbf{c}}\right)+\mathbf{g} \sin ^{2}(\pi k / 2)
$$

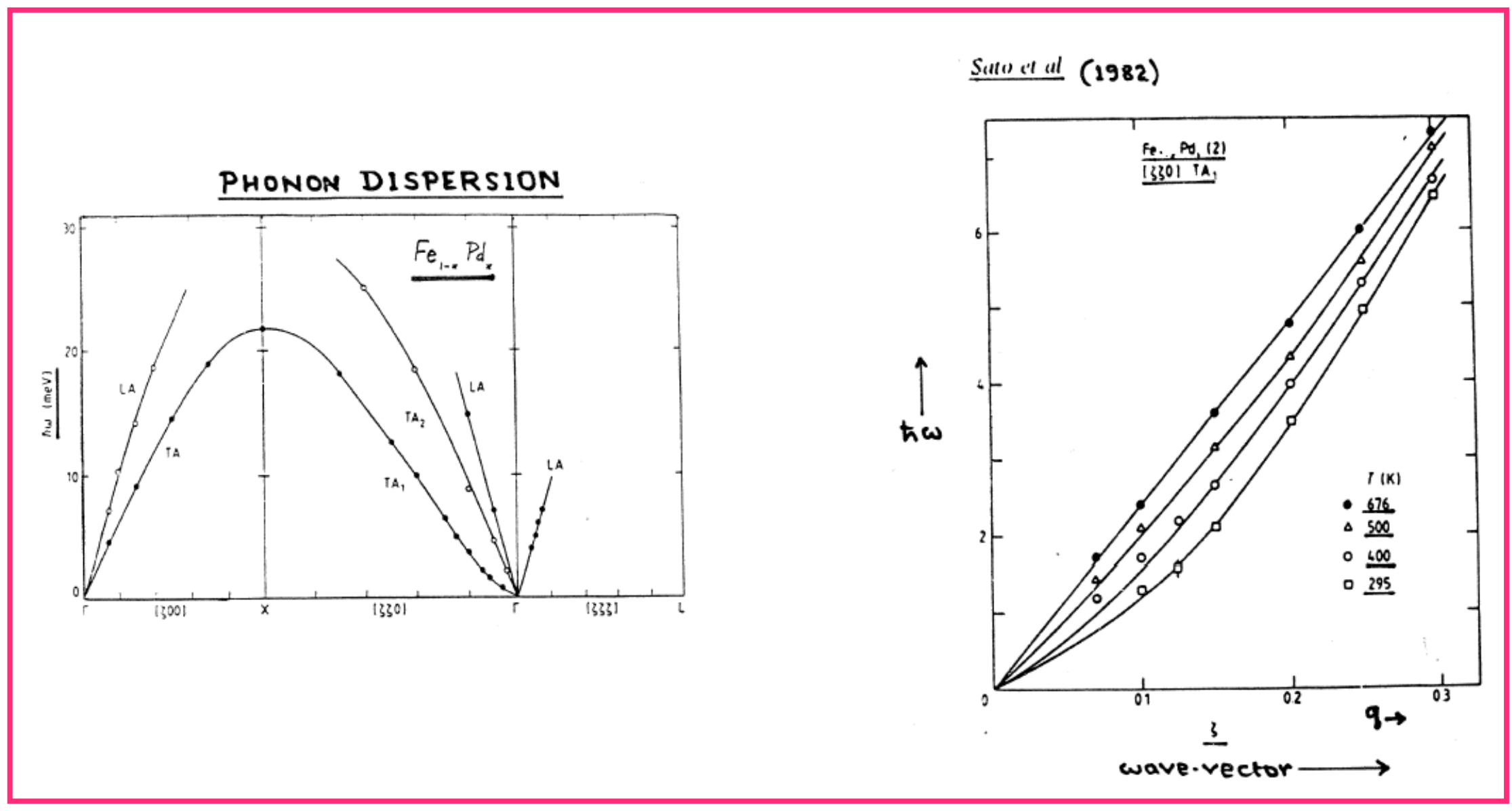




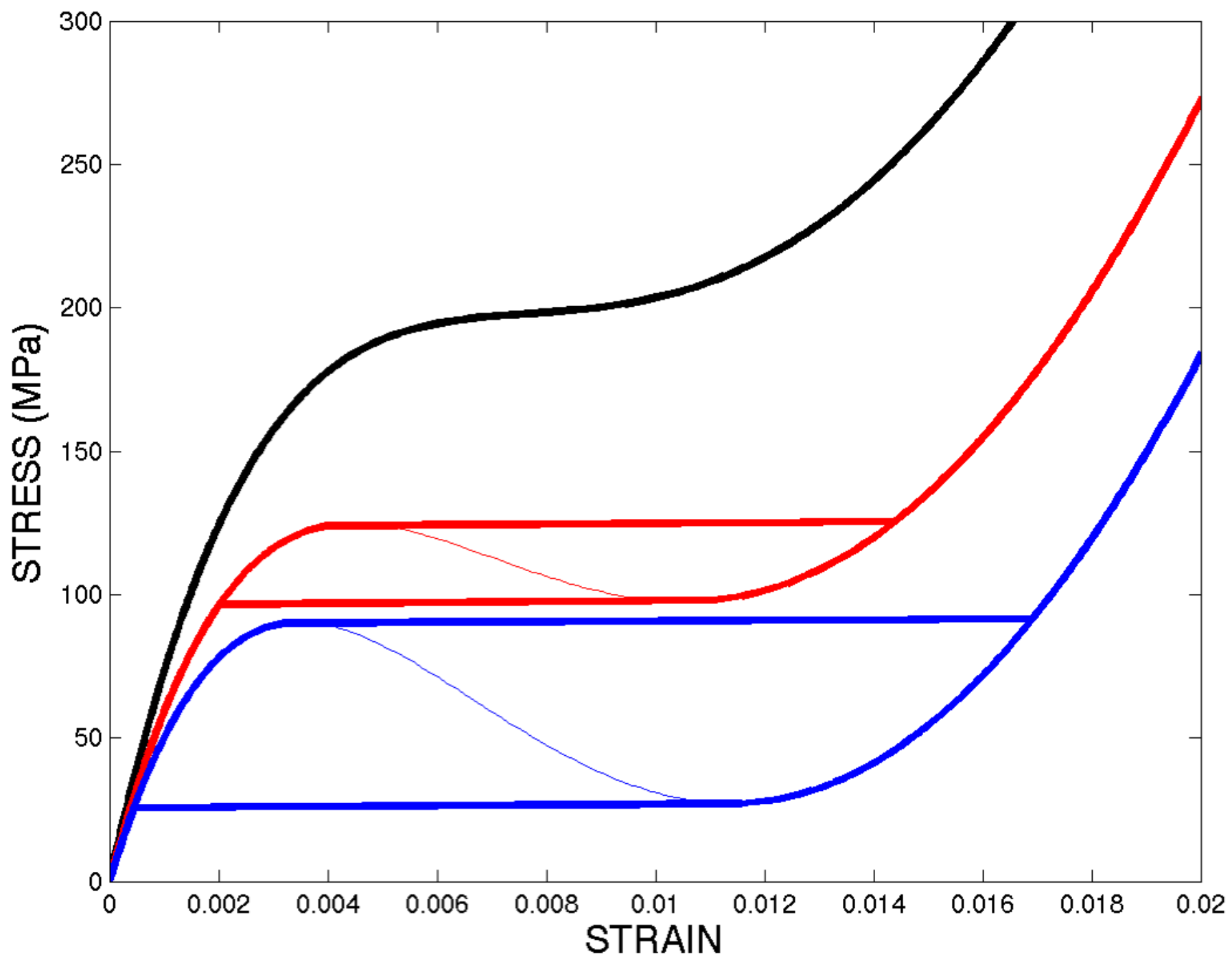




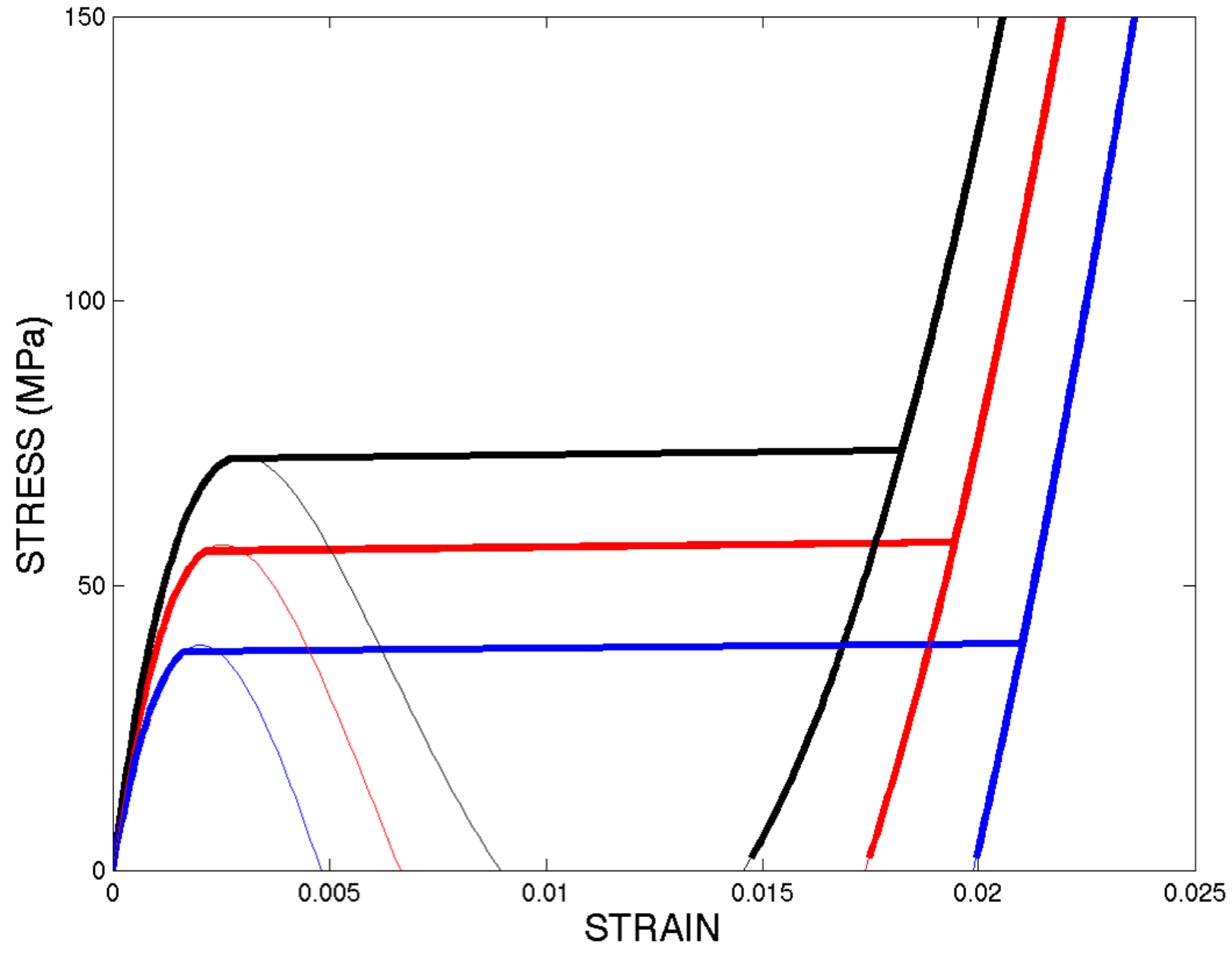


$\varepsilon_{x x}$

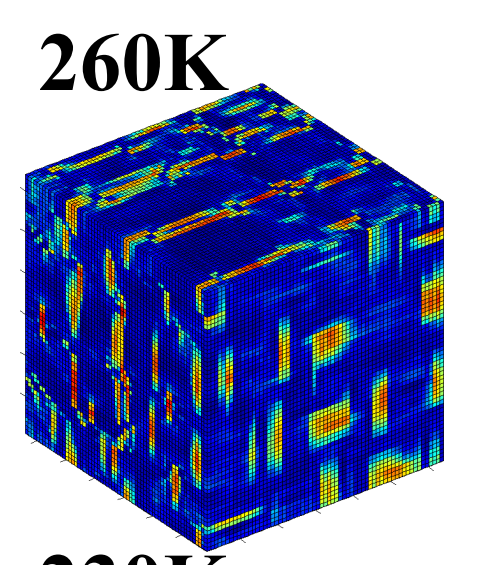

\section{K}

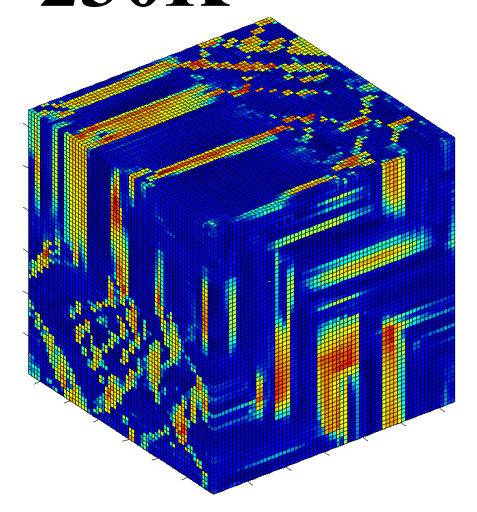

$\varepsilon_{y y}$
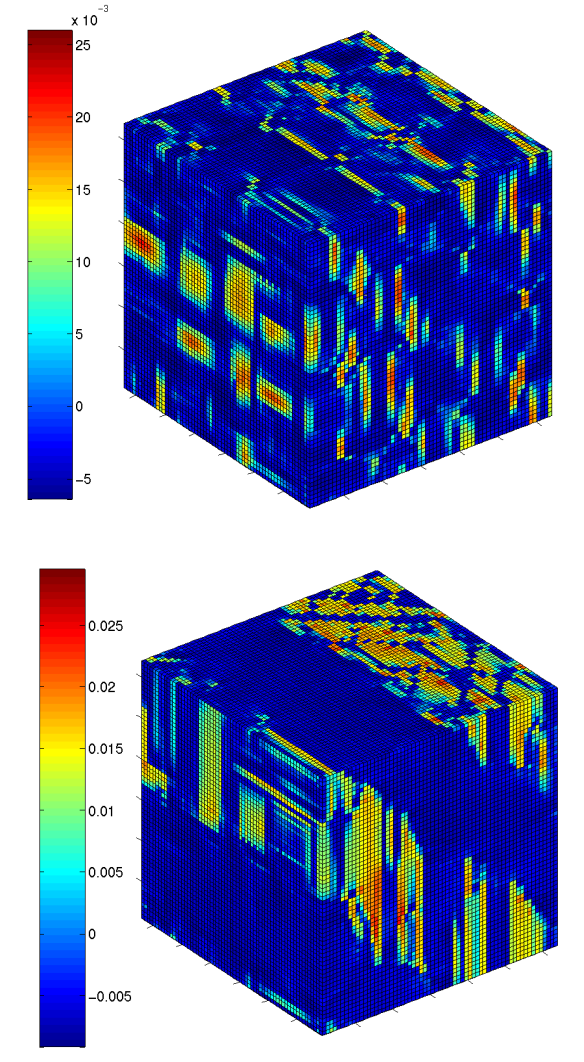

$\varepsilon_{z z}$
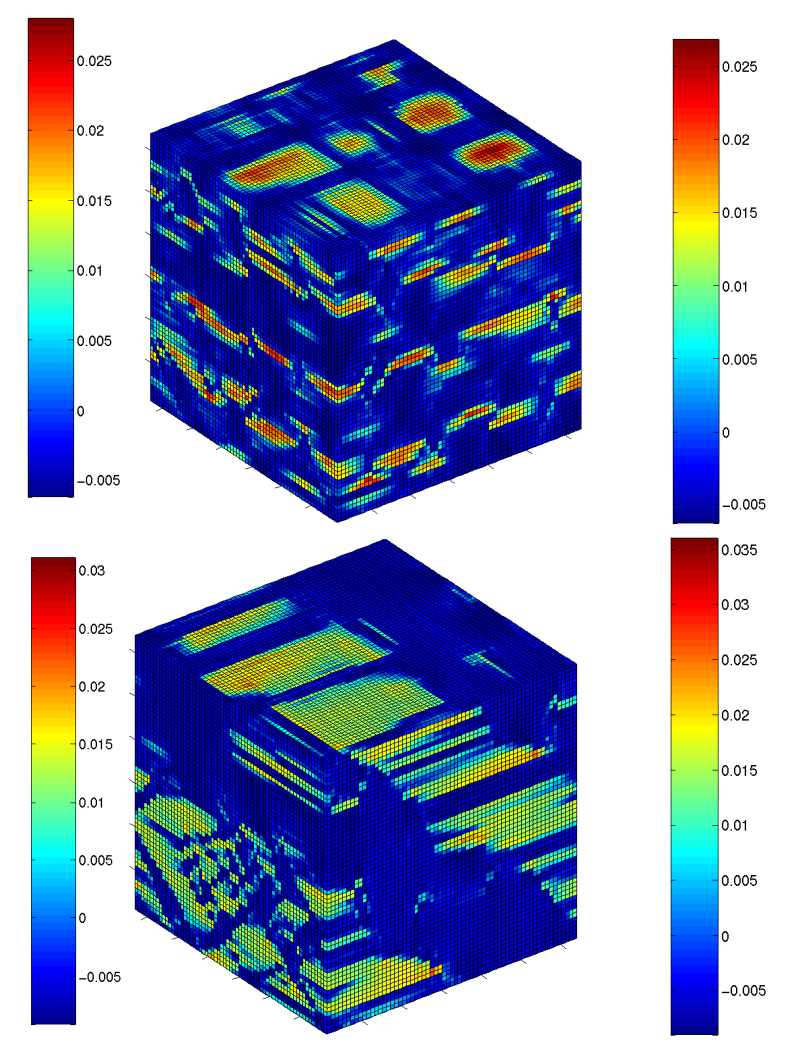

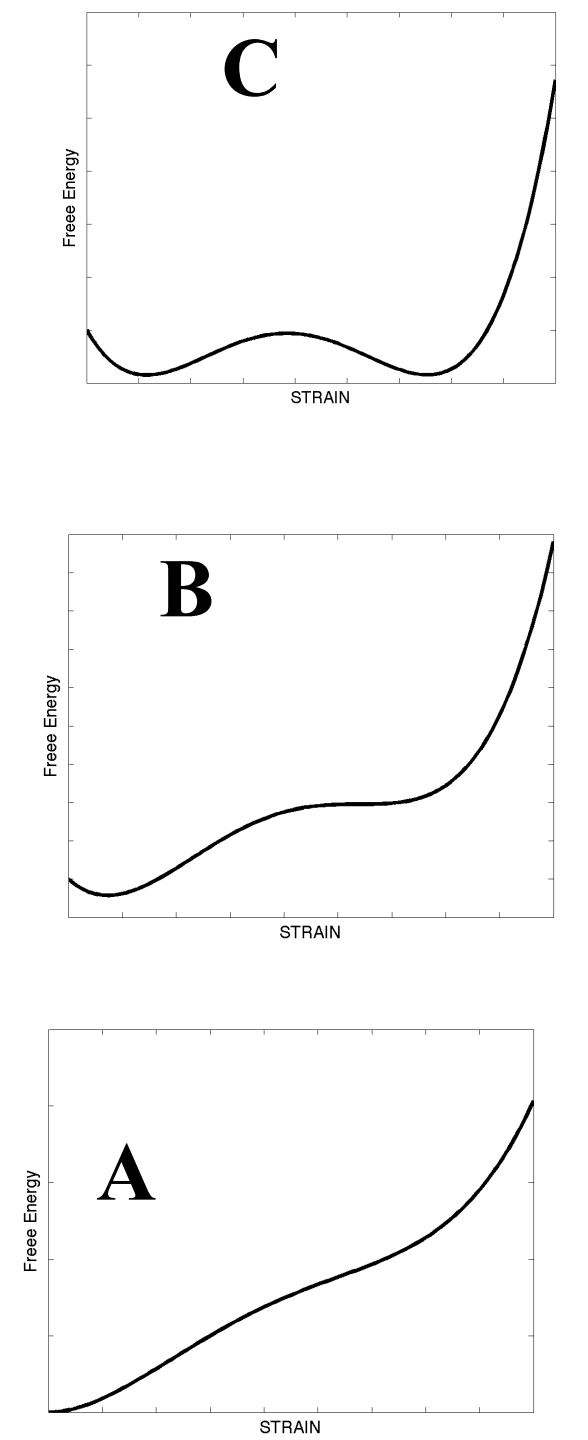
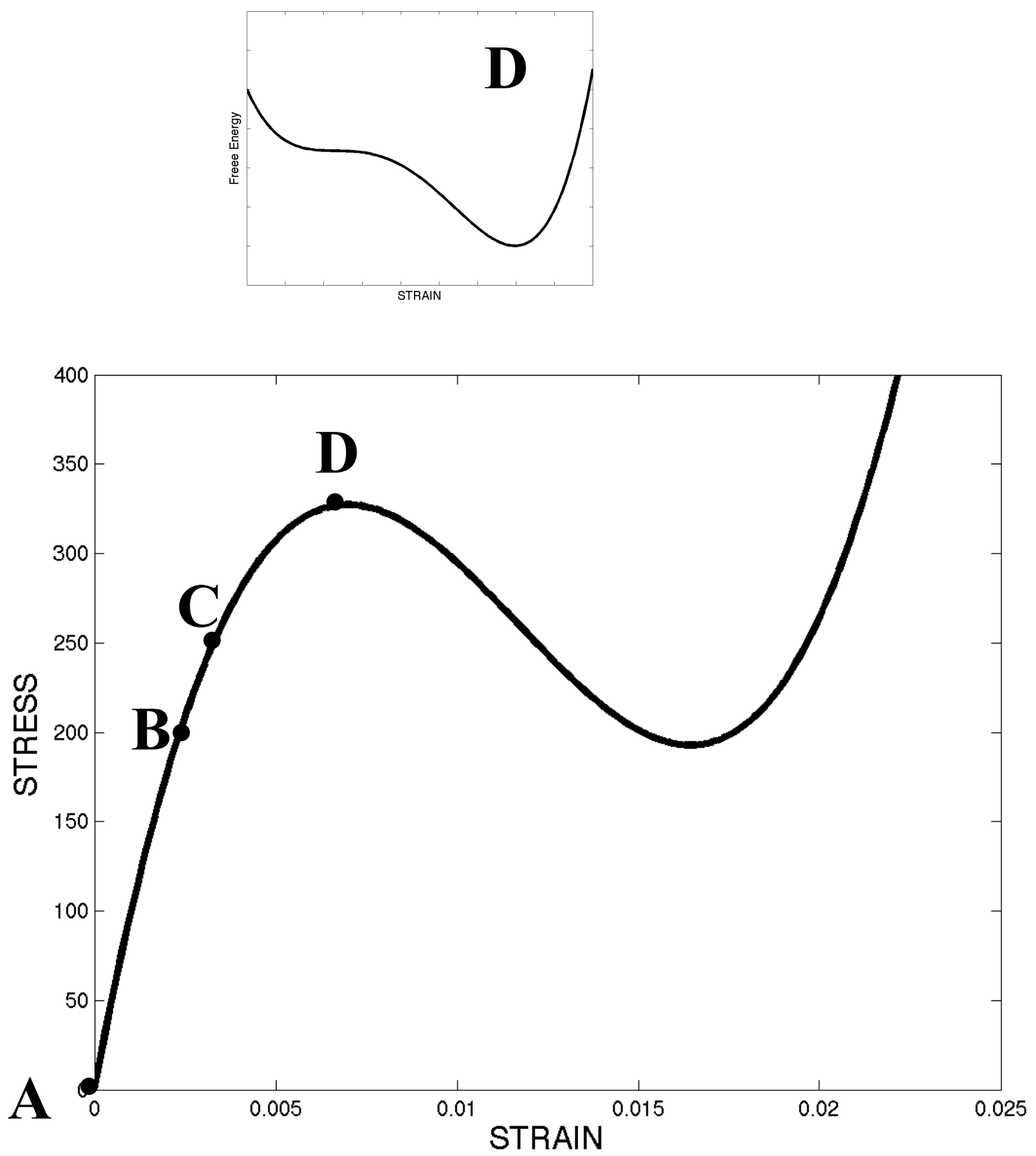


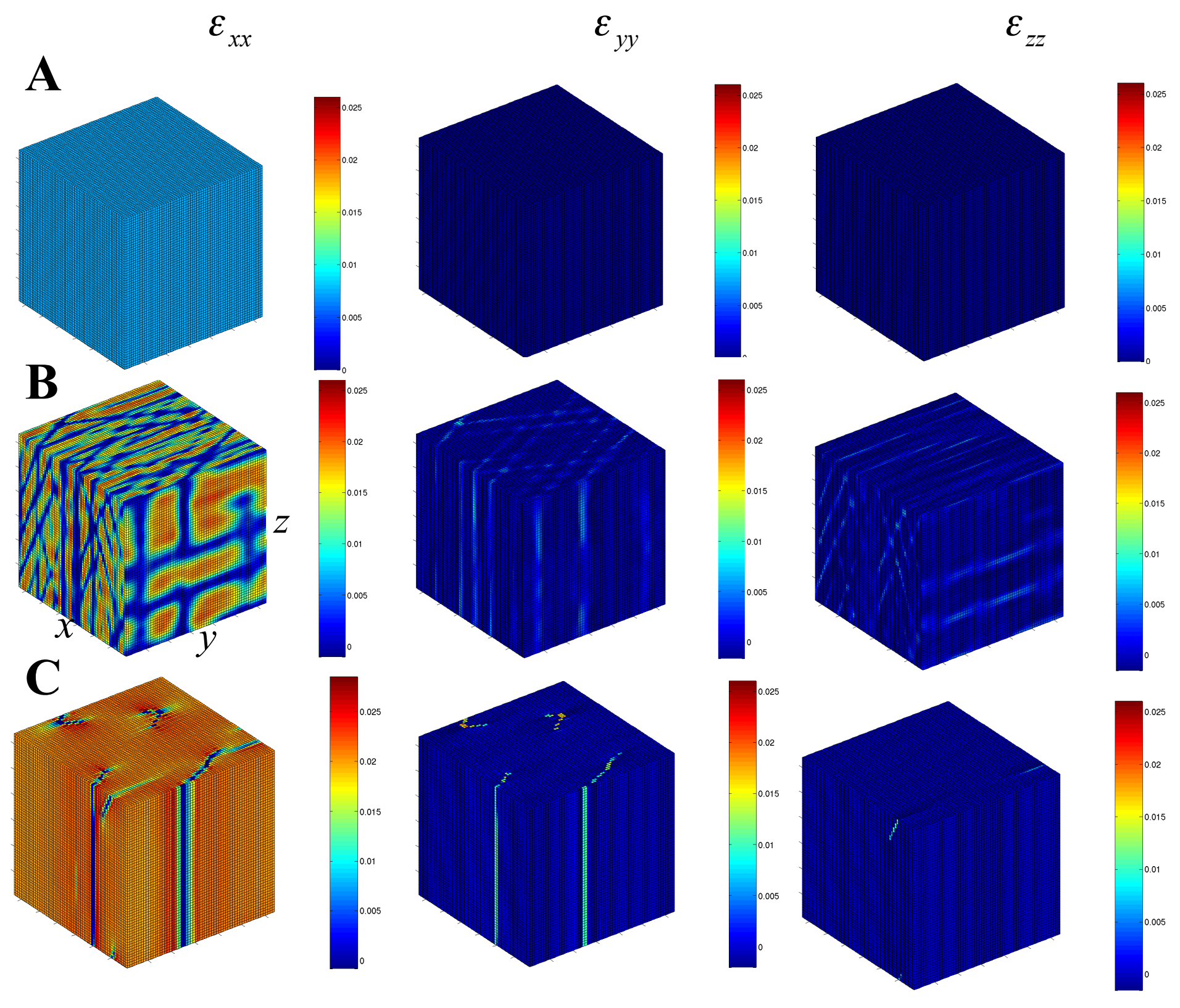




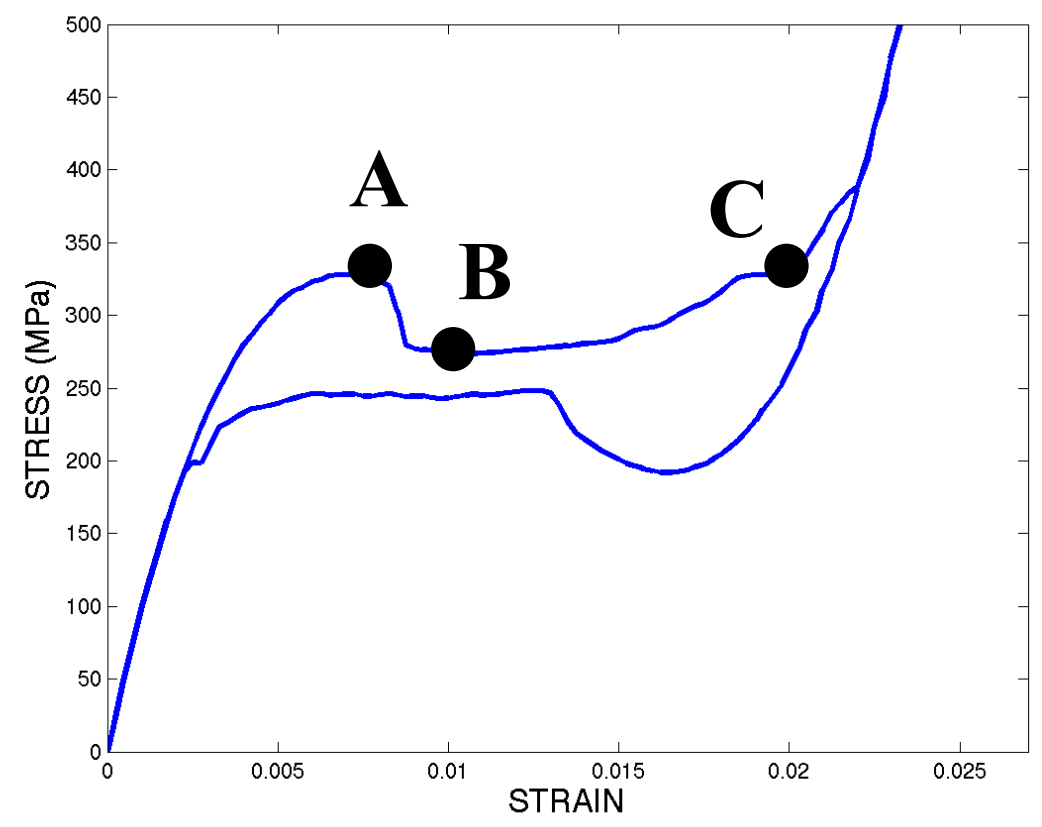




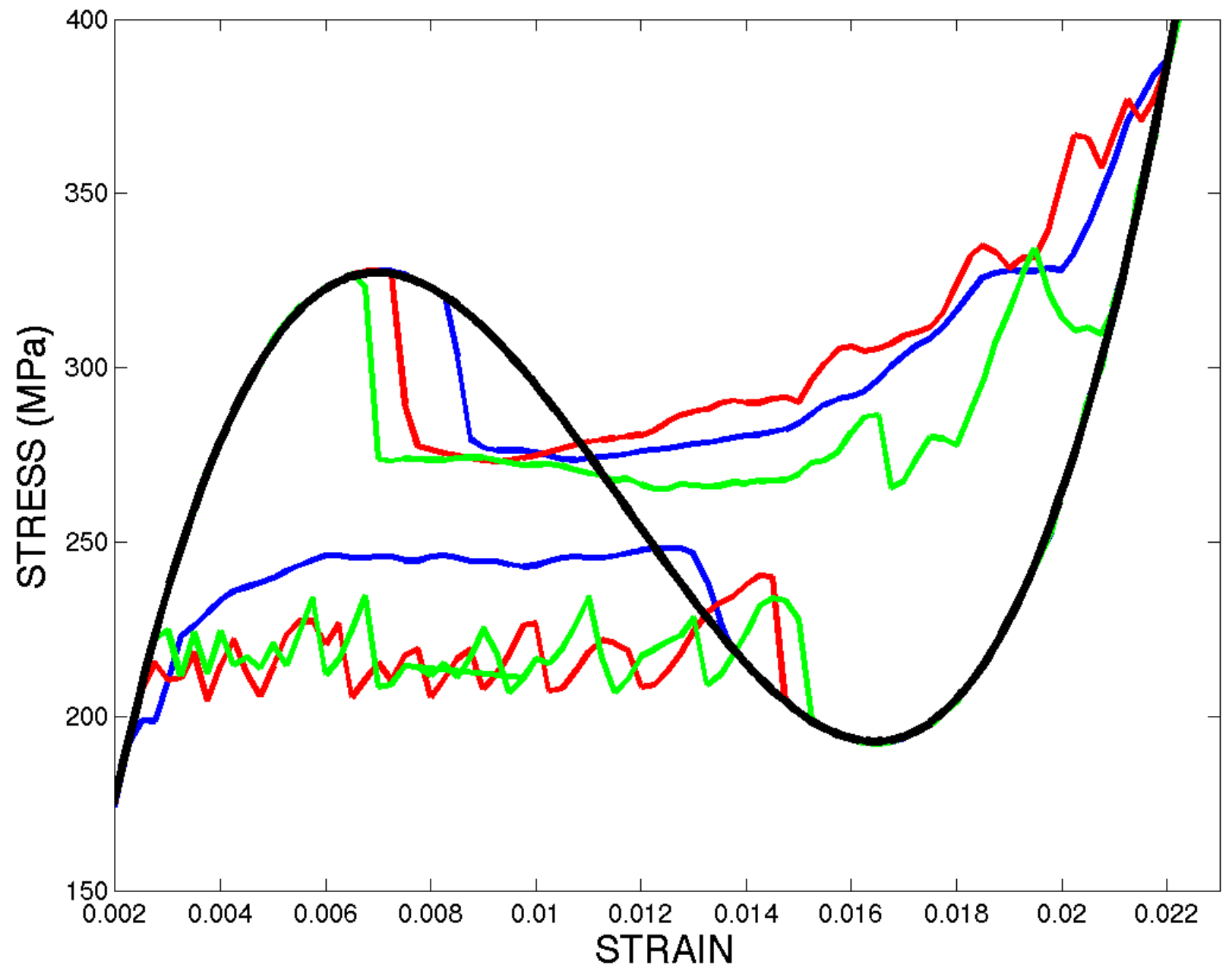


C

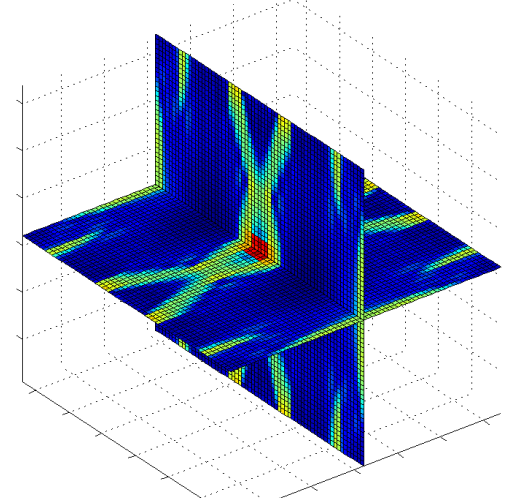

B

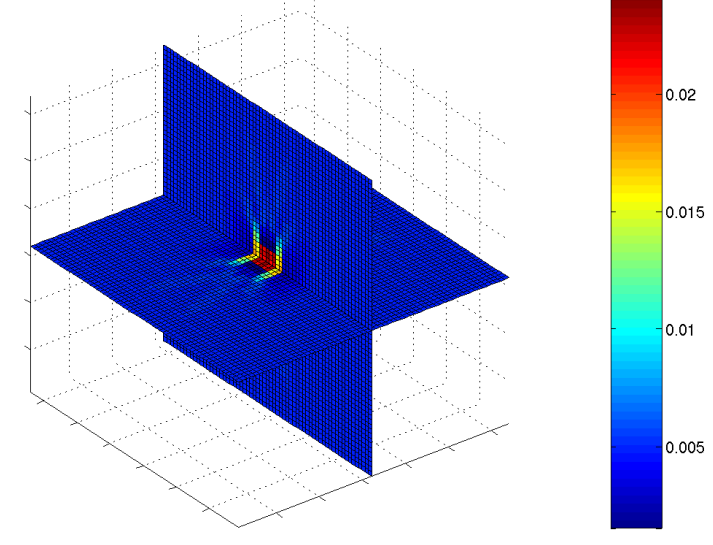

A

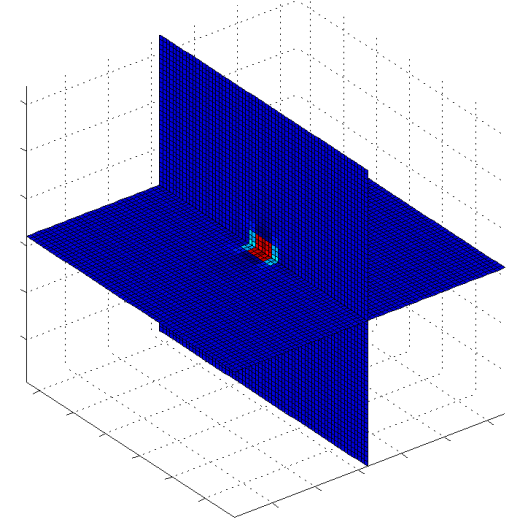

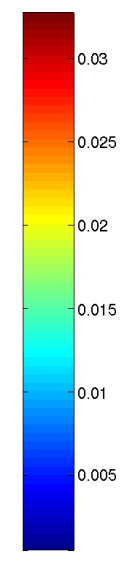

D
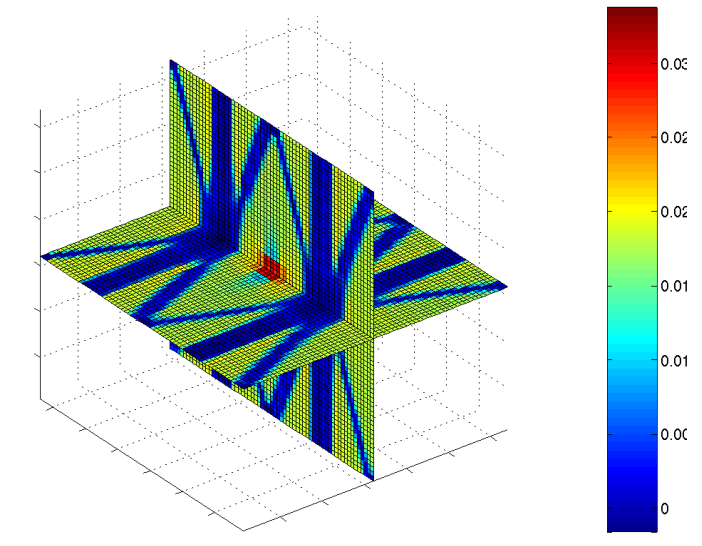

E
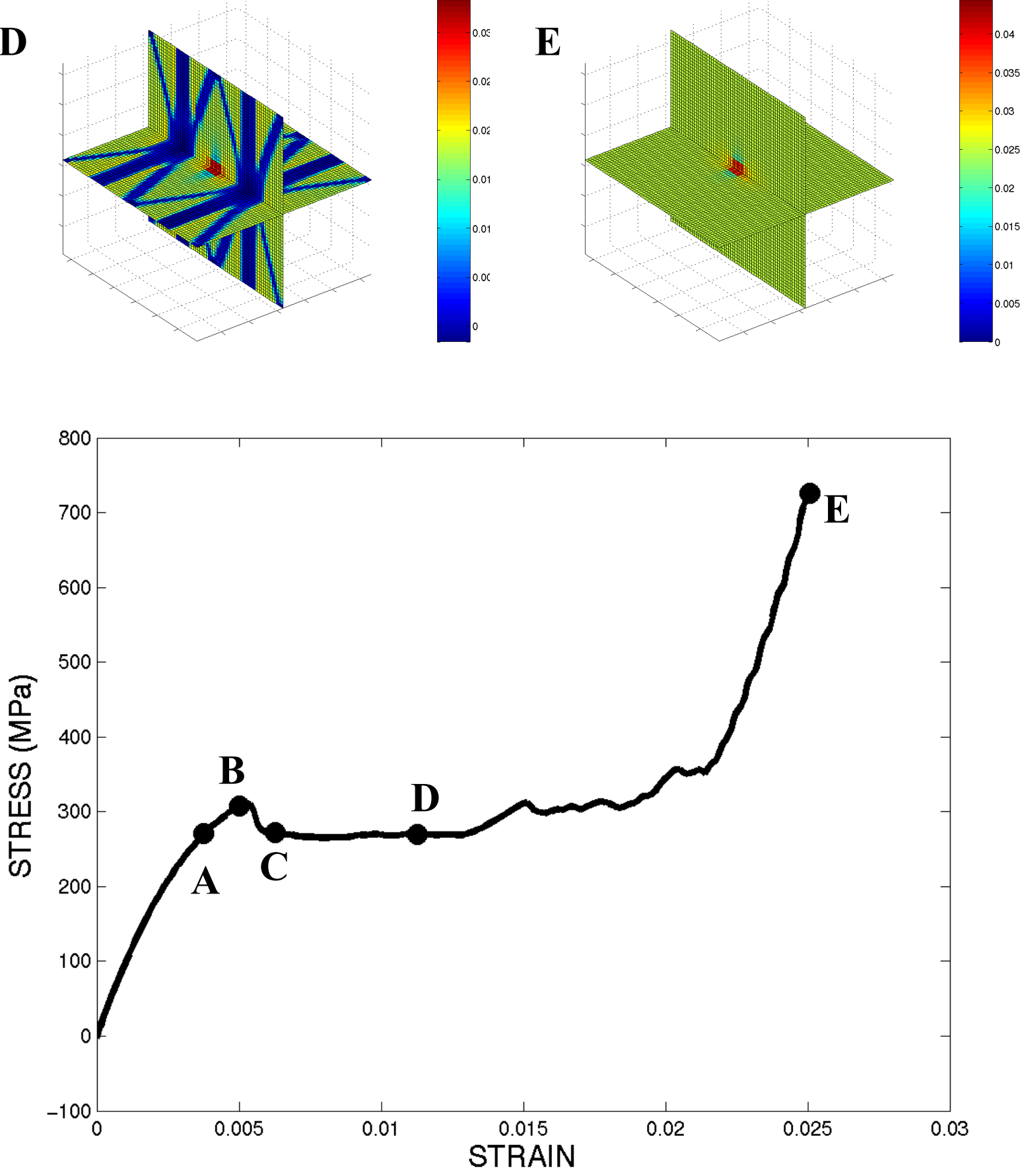
$\varepsilon_{x x}$

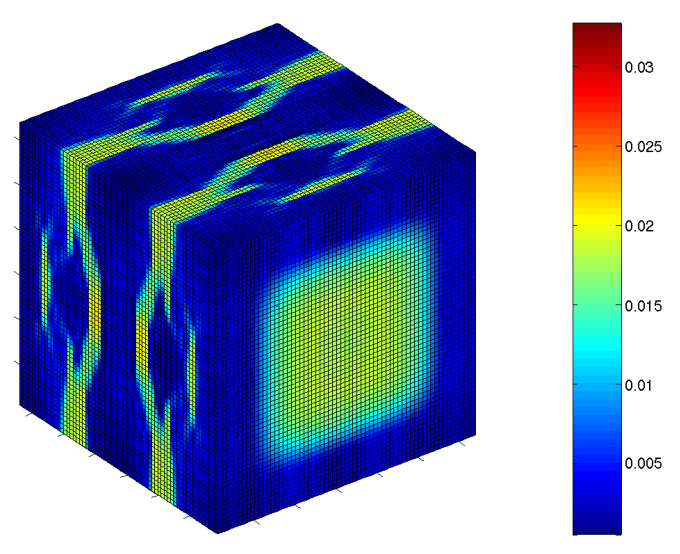

$\boldsymbol{\varepsilon}_{y y}$

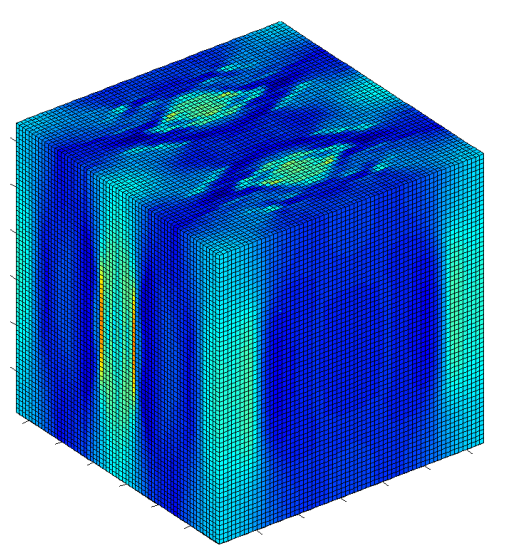

$\mathcal{E}_{z z}$

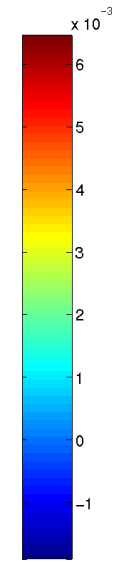

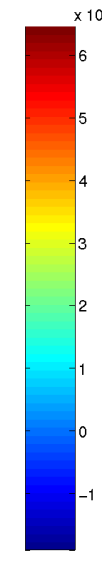




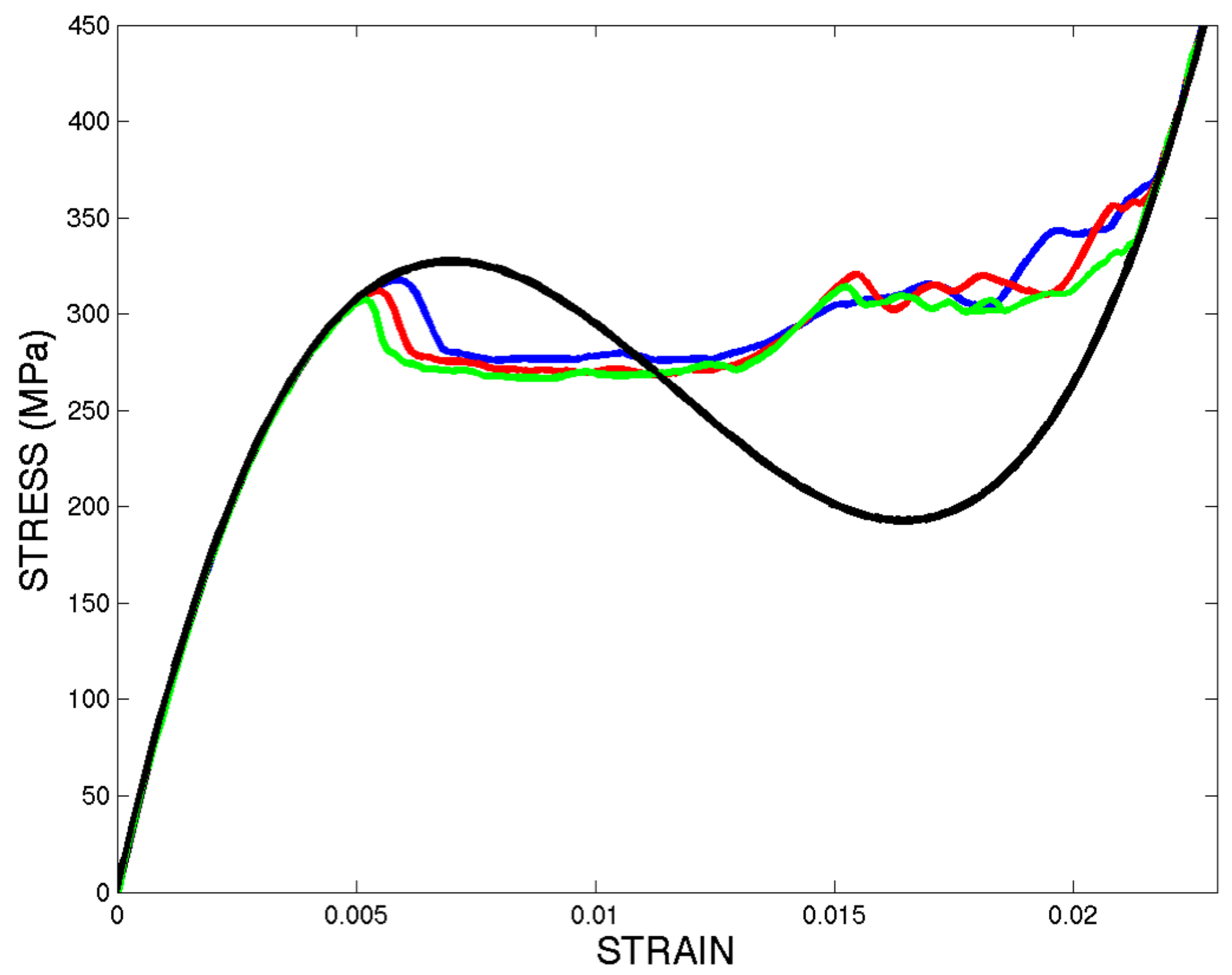




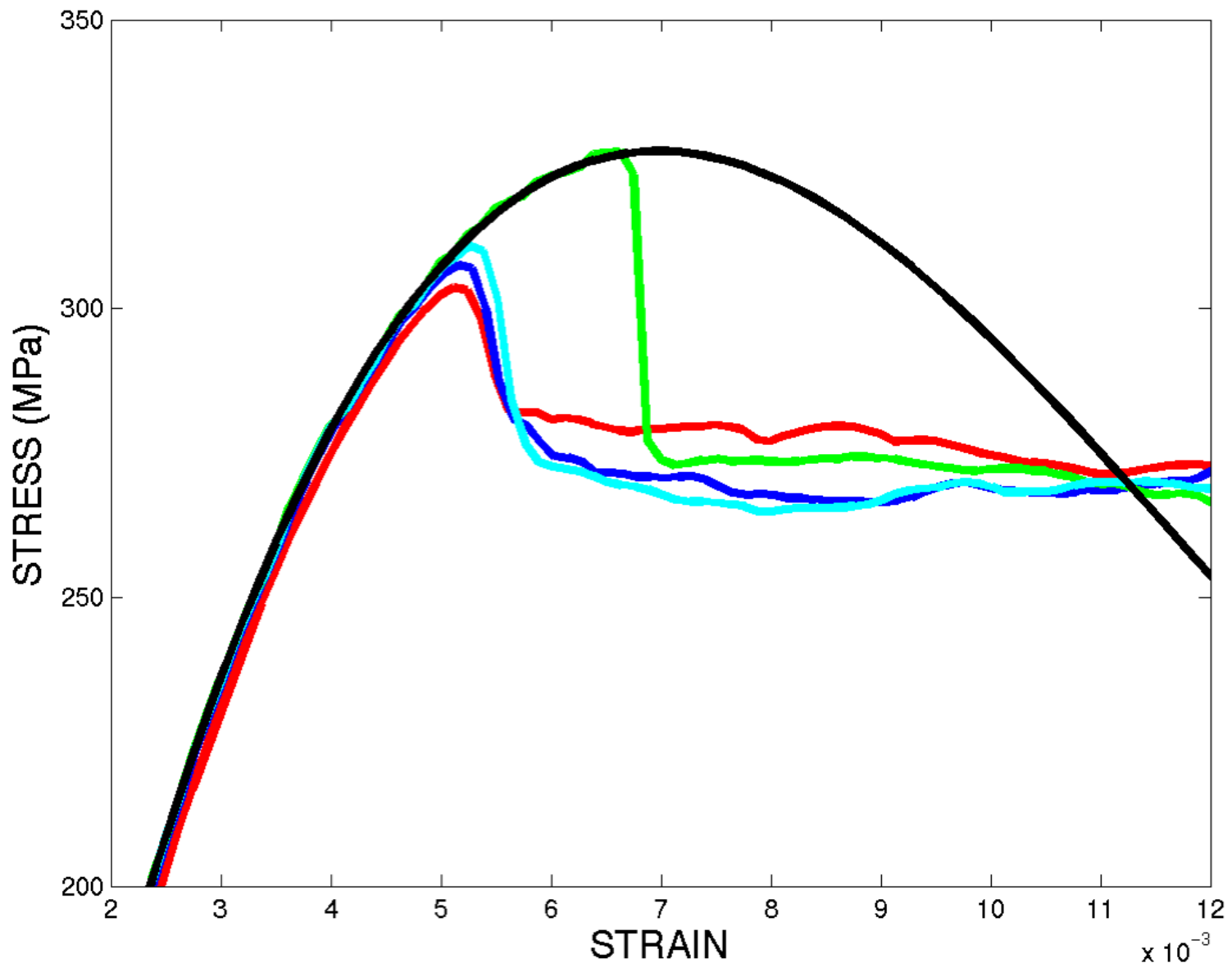


Ferroic: "crystal with two or more distinct orientation states ("domains") and switching between them"

(Aizu, 1969; Wadhawan, Intro. to ferroic materials, 2000)

ferroelectric, ferromagnetic, ferroelastic, ferrotoroidal, ferrogyrotropy,...

Symmetry breaking: Para $\rightarrow$ Ferroic

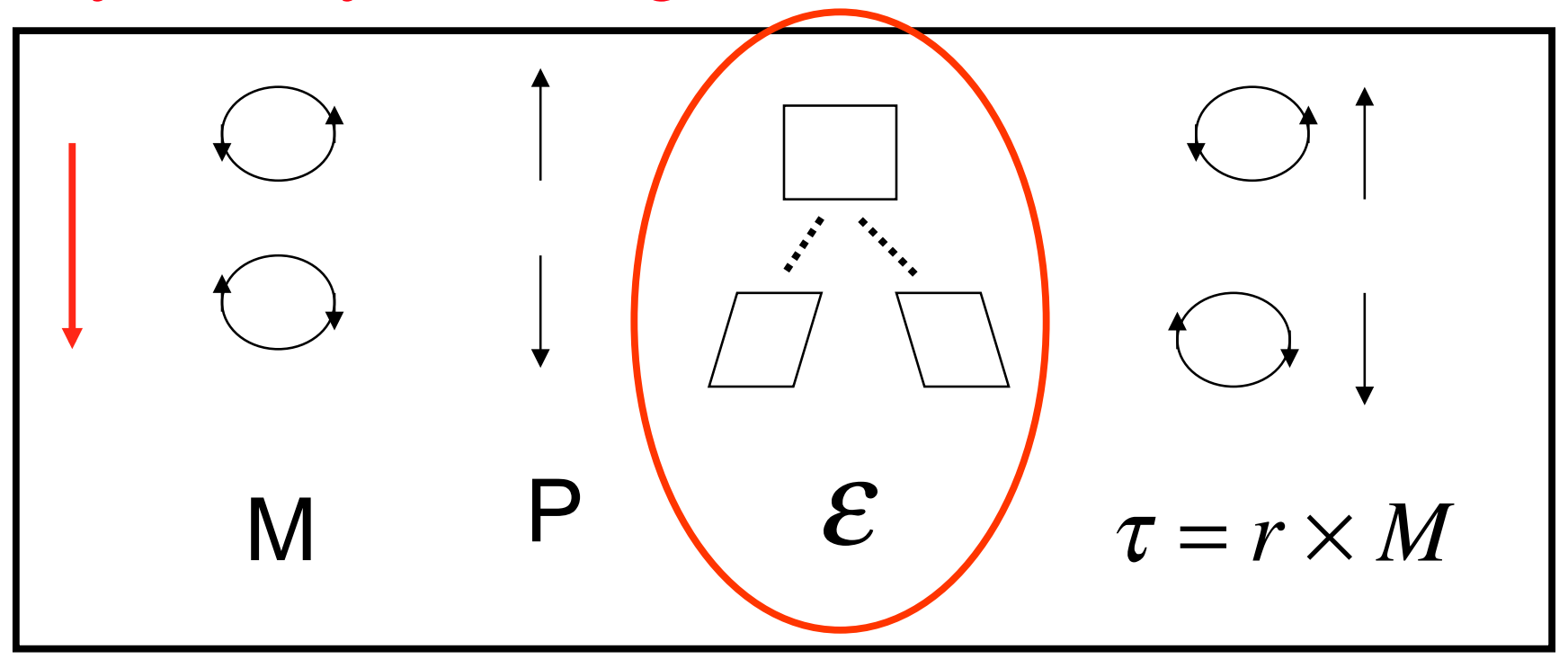


Ferroic: "crystal with two or more distinct orientation states and switching between them by external fields"

(Aizu, 69)

Multiferroic: two or more coexisting ferroic properties in the same phase

Ferromagnetic

Ferroelectric

Ferroelastic

Ferrotoroidal, ....

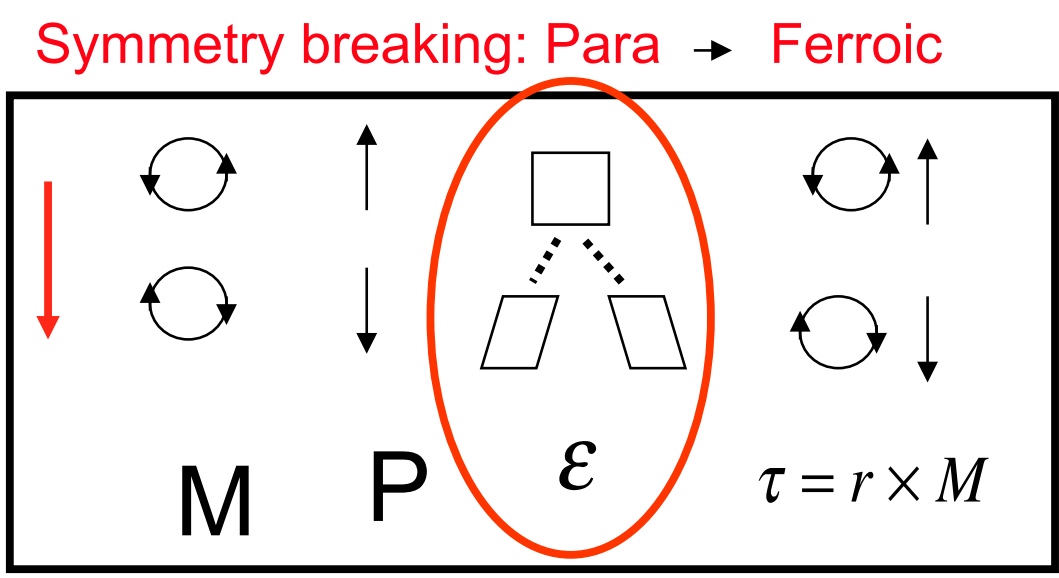

\section{Ferroelastics}

- Elastic heterogeneities: habit planes, twin boundaries :polydomains (aggregates) 


\section{Criticality and the piezoelectric response of ferroelectrics}

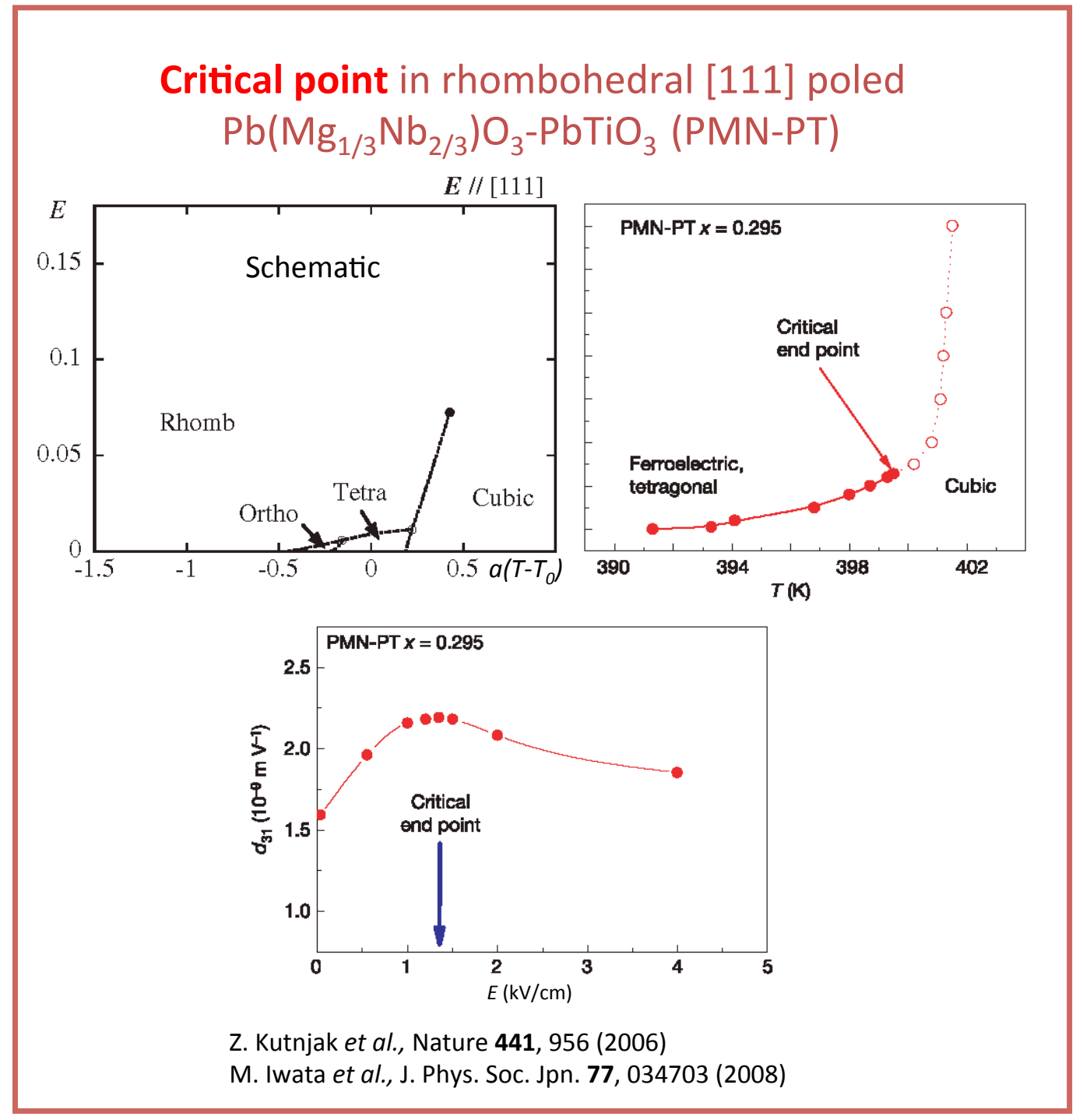

- Piezoelectric response near a critical point
Triple-tricritical point in

$$
\begin{gathered}
\mathrm{Ba}\left(\mathrm{Ti}_{0.8} \mathrm{Zr}_{0.2}\right) \mathrm{O}_{3}-\left(\mathrm{Ba}_{0.7} \mathrm{Ca}_{0.3}\right) \mathrm{TiO}_{3} \\
\text { (BZT-BCT) }
\end{gathered}
$$
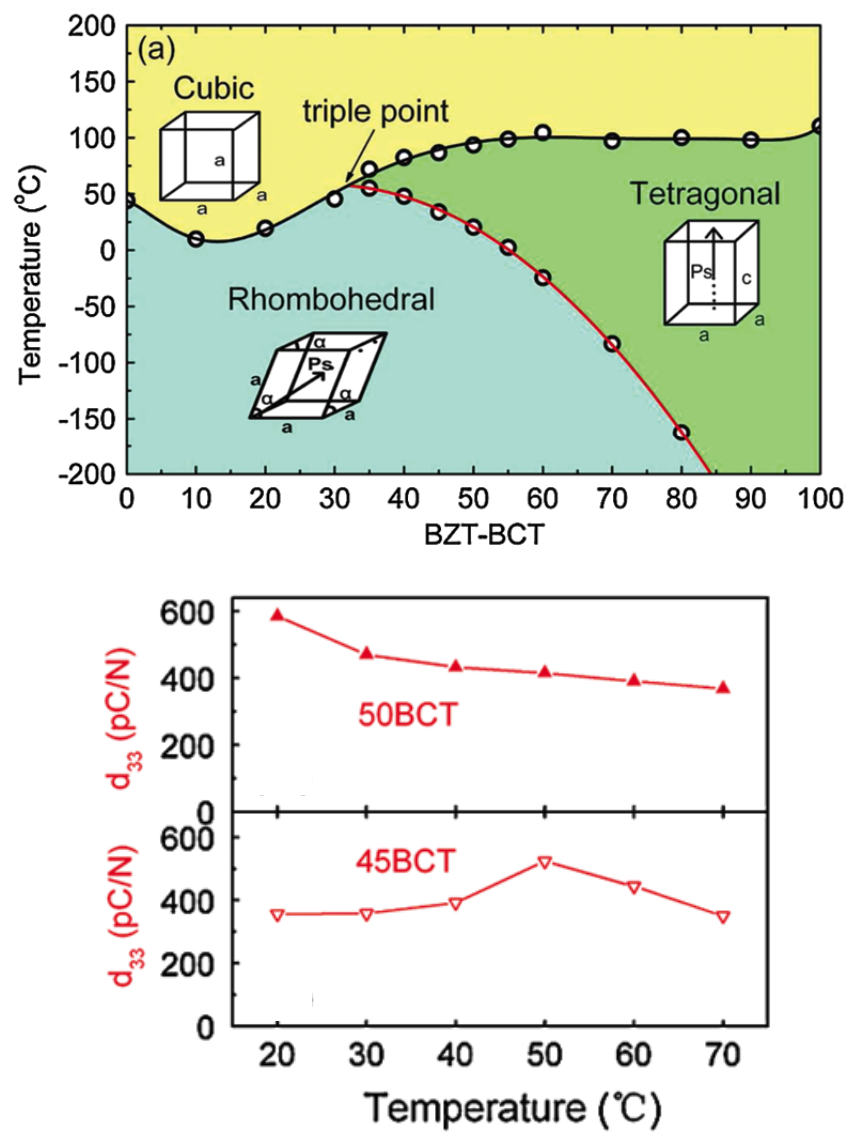

W. Liu and X. Ren, Phys. Rev. Lett. 103, 257602 (2009) 


\section{Toroidal \& Magnetoelectric Glass}

- Collusion of disorder and anisotropic, long range interactions ( $\mathrm{G}=\mathrm{E} \times \mathrm{B})$.

- Toroidal dipolar interaction short range.

- Toroidal state is more likely to be glassy; toroidal anisotropy.

- Thus pure toroidal order more difficult to observe directly: $\mathrm{LiCO}\left(\mathrm{PO}_{4}\right)_{3}$.

- Multiglass: $\mathrm{Fe}_{2} \mathrm{TiO}_{5}$ (S. Sharma et al., PRB 2014).

- Simulations with short range interaction. 


\section{MAGNETOELECTRIC COOLING BY ME $_{H}$ IN NMTO}

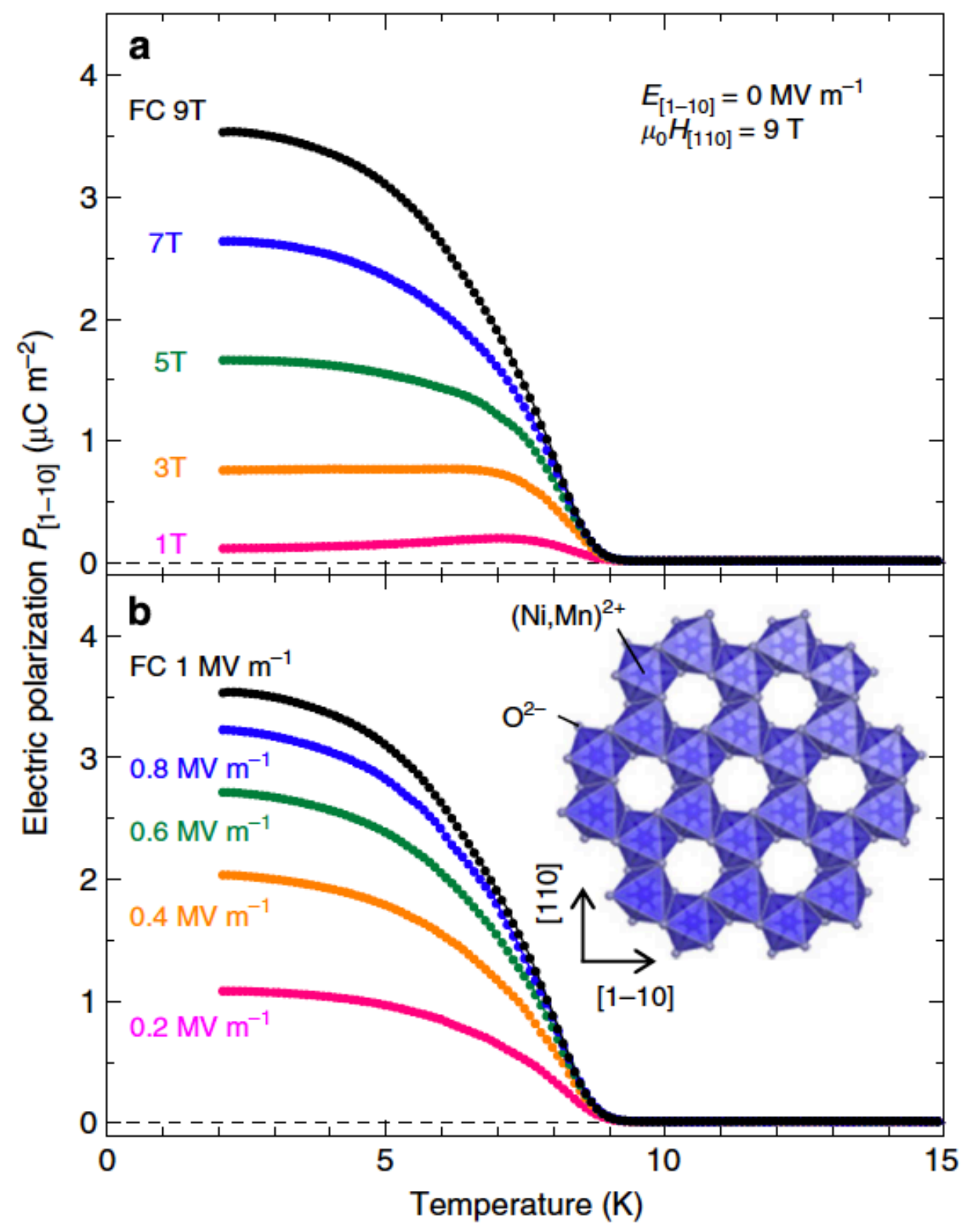

Y. Yamaguchi And T. Kimura, Nature Commun. June (2013)

$\mathrm{Ni}_{0.4} \mathrm{Mn}_{0.6} \mathrm{TiO}_{3}$ 


\section{TOROIDAL FROZEN STATE: MAGNETOELECTRIC CONTROL}

a

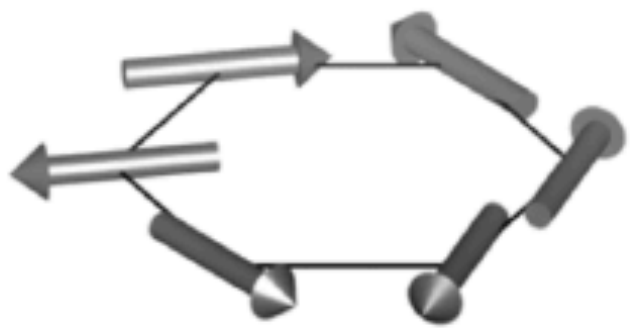

b

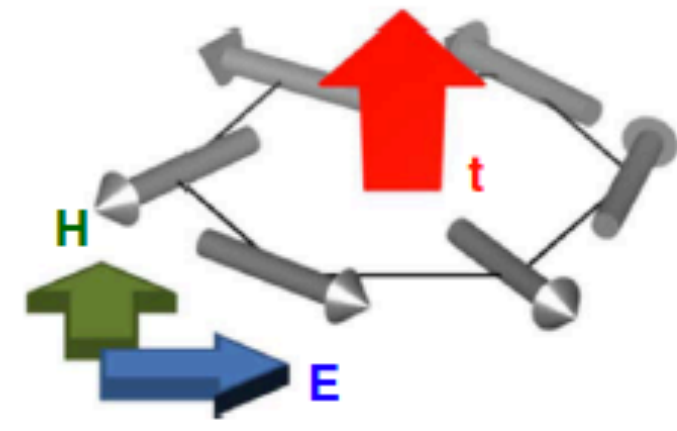

C

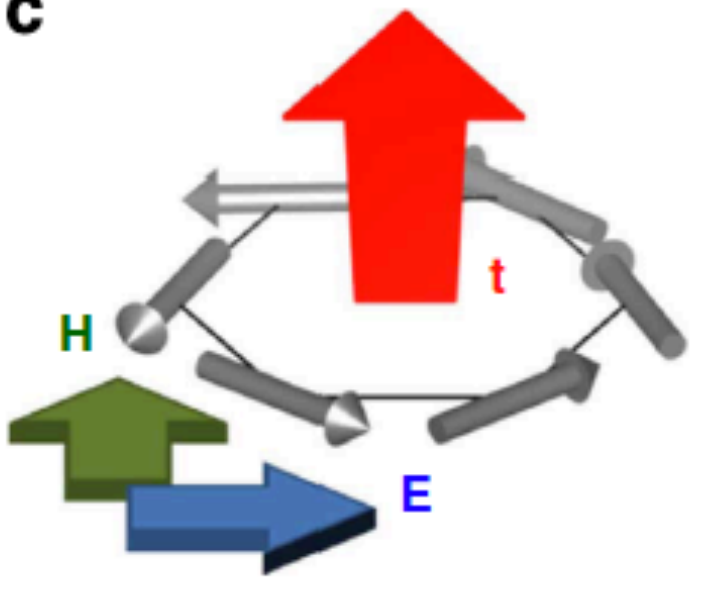

d

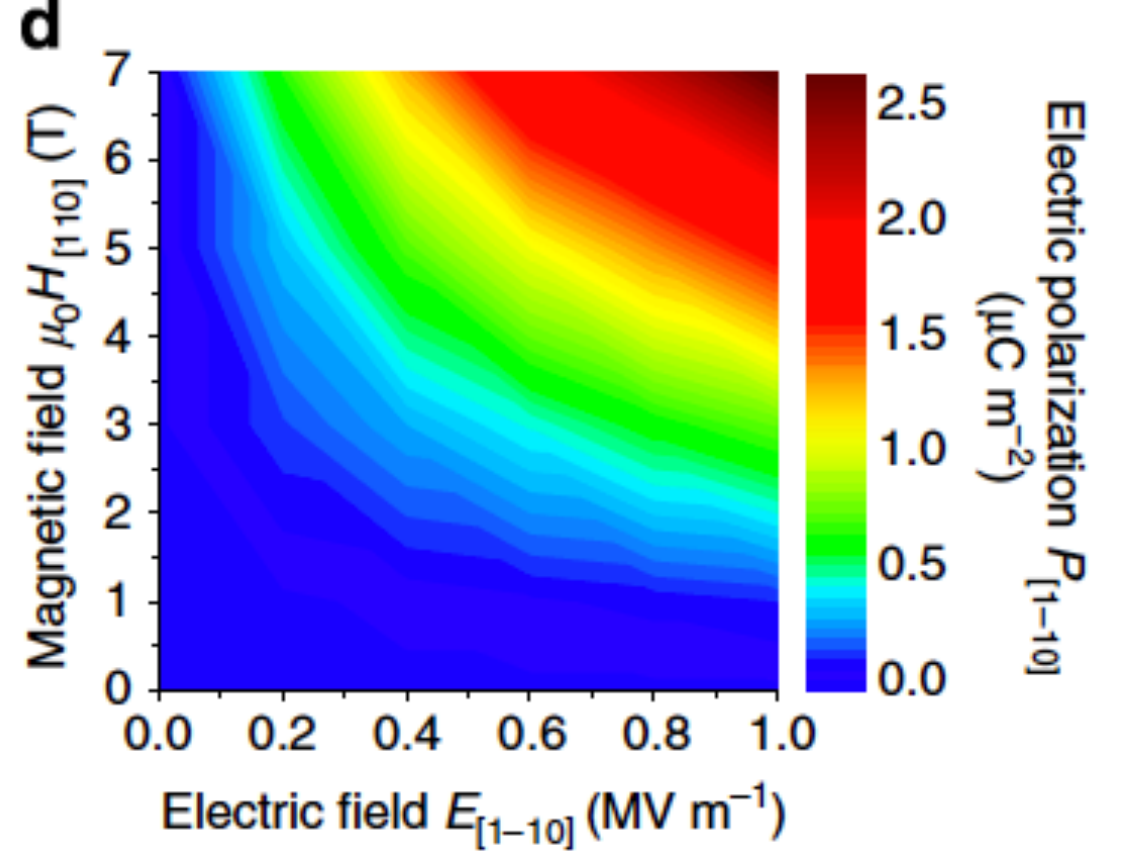

e

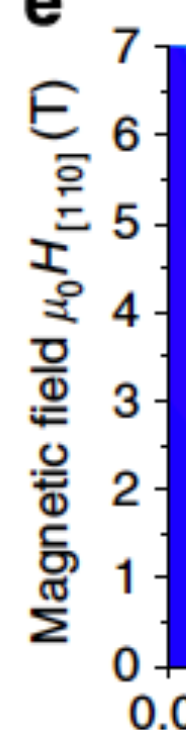

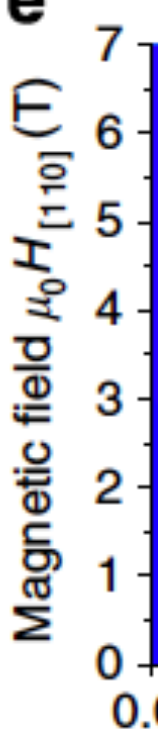

2.5 莨 1.5 1.0 두응 0.5 足 Electric field $E_{[1-10]}\left(\mathrm{MV} \mathrm{m}^{-1}\right)$ 